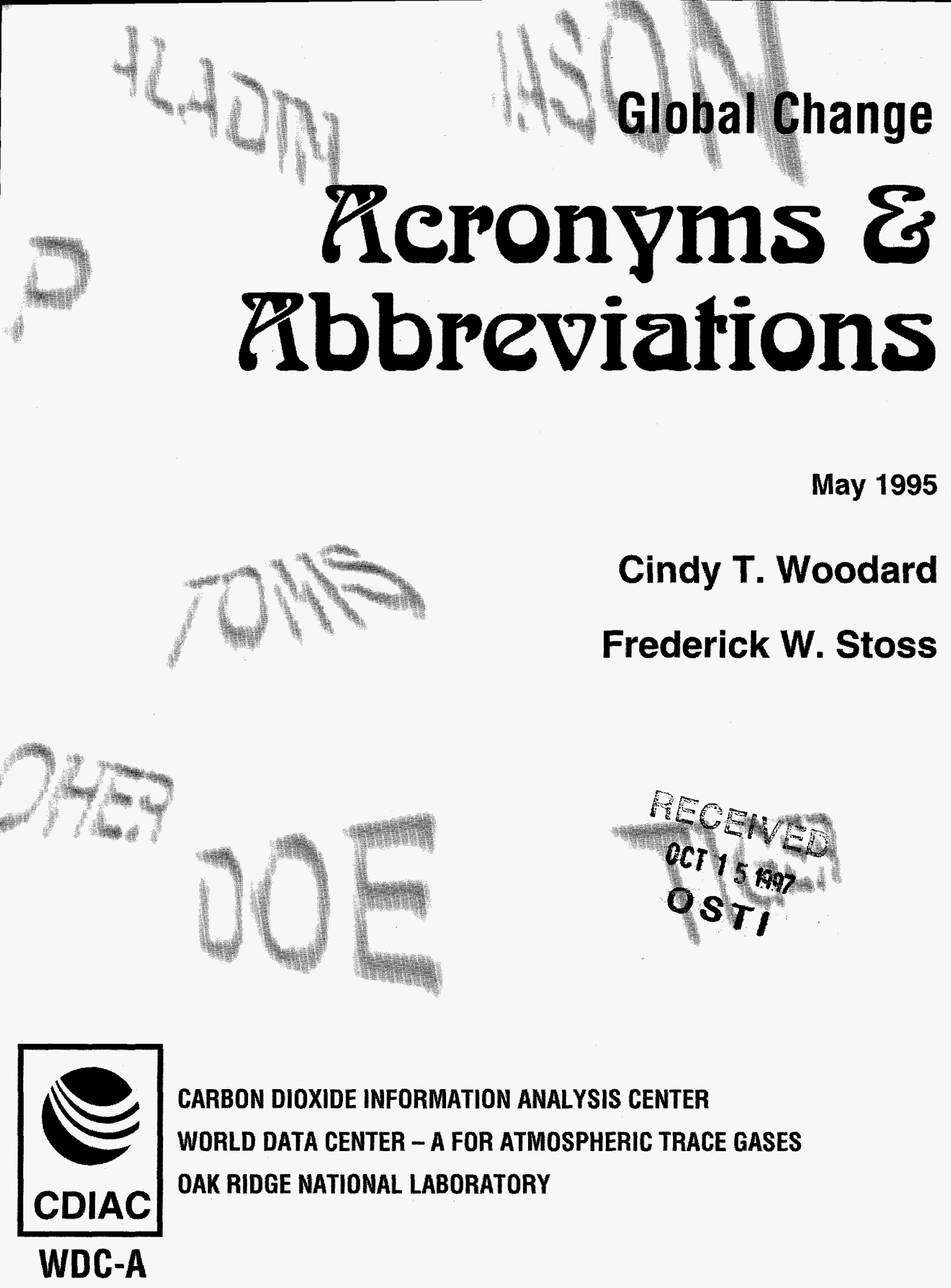


Available from the Carbon Dioxide Information Analysis Center, P.O. Box 2008, Oak Ridge, TN 37831-6335 at no cost.

This report has been reproduced directly from the best available copy.

Available to DOE and DOE contractors from the Office of Scientific and Technical Information, P.O. Box 62, Oak Ridge, TN 37831; prices available from (615) 576-8401.

Available to the public from the National Technical Information Service, U.S. Department of Commerce, 5285 Port Royal Road, Springfield, VA 22161.

This report was prepared as an account of work sponsored by an agency of the United States Government. Neither the United States Government nor any agency thereof, nor any of their employees, makes any warranted, express or implied, or assumes any legal liability or responsibility for the accuracy, completeness, or usefulness on any information, apparatus, product, or process disclosed, or represents that its use would not infringe privately owned rights. Reference herein to any specific commercial product, process, or service by trade name, trademark, manufacturer, or otherwise, does not necessarily constitute or imply its endorsement, recommendation, or favoring by the United States Government or any agency thereof. The views and opinions of authors expressed herein do not necessarily state or reflect those of the United States Government or any agency thereof. 


$$
\text { ORNL/CDIAC }-98001235
$$

Carbon Dioxide Information Analysis Center

World Data Center-A for Atmospheric Trace Gases

Oak Ridge National Laboratory

\author{
gLOBAL Change
}

\title{
ACIRDNYMS AND AIBIBIRIEVIIATIIONS
}

May 1995

\section{Cindy T. Woodard}

and

Frederick W. Stoss*

Carbon Dioxide Information Analysis Center

Environmental Sciences Division

Oak Ridge National Laboratory

*Energy, Environment and Resources Center

The University of Tennessee, Knoxville

Environmental Sciences Division

Publication No. 4415

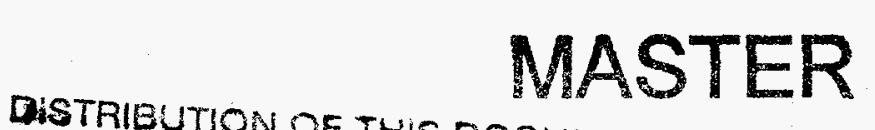




\section{DISCLAMIER}

Portions of this document may be illegible in electronic image products. Images are produced from the best available original docoment. 
FOREWORD $\quad$ v

ABSTRACT vii

$\begin{array}{ll}\text { ACKNOWLEDGMENTS } & \text { ix }\end{array}$

APPENDIX A. CARBON DIOXIDE INFORMATION ANALYSIS CENTER 71

$\begin{array}{ll}\text { APPENDIX B. CDIAC ORDER FORM } & 75\end{array}$ 

The environmental literature of science and policy is filled with numerous acronyms and abbreviations that for the uninitiated seemingly represent a jargon unto itself. It is often frustrating and sometimes embarrassing to be involved in a scholarly discussion when participants have a raft of acronyms rolling off their tongues and speak with such authority, all the while assuming everyone knows what they mean. The listener is left wondering what these experts just tried to say. Of course, the same holds true for scholarly works peppered with these capitalizations as well. It is no wonder that the cross-disciplinary exchange of ideas can be such a baffling experience!

In the global change community, acronyms and abbreviations are abundant. Program names, instruments, agencies, geographic reference points, and administrative bodies all are represented with unique and sometimes clever aggregations of letters.

This inventory of Global Change Acronyms and Abbreviations greatly expands the short list of 60 entries included in the Glossary: Carbon Dioxide and Climate [1], produced by the Carbon Dioxide Information Analysis Center. This current document consolidates acronyms and abbreviations from many disciplines: agriculture, atmospheric science, ecology, environmental science, oceanography, policy science, and other fields.

Items included in this first edition of Global Change Acronyms and Abbreviations have been collected from a number of sources: technical reports, policy documents, global change program announcements, newsletters, and other periodicals. In addition to the hard-copy version of this document, this list of acronyms and abbreviations is available in DOS-formatted diskettes and in CDIAC's anonymous File Transfer Protocol (FTP) area on the Internet.

Included in this work are abbreviations [e.g., Carbon Dioxide Information Analysis Center (CDIAC), Oak Ridge National Laboratory (ORNL)], and acronyms-words formed from the letters in a series of related words [e.g., Terrestrial Initiative in Global Environmental Research (TIGER), Tropical Ocean and Global Atmosphere (TOGA)].

All entries are arranged in alphabetical order with lower case entries appearing first (i.e., aa before AA) and unpunctuated entries appearing before punctuated entries (i.e., AA before A.A.). Foreign acronyms are included in this document. The language of origin and the English equivalent are provided when possible. If the definition in the language of origin is not available, the English equivalent is provided.

Every effort will be made to keep this reference tool current. Hard copy editions will be revised, and the electronic versions on diskette and on the Internet will also be routinely updated prior to distribution. This document is intended to be a dynamic work, changing as new acronyms and abbreviations arise through the creative talents of bureaucratically driven science and policy personnel. We invite you to make this your document by sending us new entries. 
Individuals responsible for preparing written and oral presentations are encouraged to use this document as an aid for defining acronyms and abbreviations. The editorial standard is to define the acronym or abbreviation (usually as a parenthetic note) the first time it appears in the text. If a lengthy document or report is prepared in which many acronyms and abbreviations appear, a "List of Acronyms and Abbreviations" is commonly appended to the work. The identification of acronyms and abbreviations is a value-added consideration for which many readers will be grateful.

It is hoped you, as readers, benefit from this work. As compilers, we delight in our knowledge that the end of our quest is not in sight.

Cindy Woodard

Fred Stoss

[1] Glossary: Carbon Dioxide and Climate, ORNL/CDIAC-39, F.M. O'Hara, Jr. Carbon Dioxide Information Analysis Center, Oak Ridge National Laboratory. 1990. 
Woodard, C.T., and F.W. Stoss. 1994. Global Change Acronyms and Abbreviations. ORNL/CDIAC-83 and Environmental Sciences Publication No. 4415. Carbon Dioxide Information Analysis Center, Oak Ridge National Laboratory, Oak Ridge, Tennessee. 77pp.

This list of acronyms and abbreviations is compiled to provide the user with a ready reference to decipher the linguistic initialisms and abridgements for the study of global change. The terms included in this first edition were selected from a wide variety of sources: technical reports, policy documents, global change program announcements, newsletters, and other periodicals. The disciplinary interests covered by this document include agriculture, atmospheric science, ecology, environmental science, oceanography, policy science, and other fields. In addition to its availability in hard copy, the list of acronyms and abbreviations is available in DOS-formatted diskettes and through CDIAC's anonymous File Transfer Protocol (FTP) area on the Internet.

Key words: Abbreviations, acronyms, agriculture, air pollution, atmospheric chemistry, carbon dioxide, climate change, climatology, chlorofluorocarbons, Earth atmosphere, ecology, global warming, greenhouse effect, meteorology, and oceanography. 

We wish to thank a number of people in the preparation of this report. We would like to thank Bob Sepanski and Russ Vose, both data analysts at CDIAC, for their technical review of this manuscript and for their comments and suggestions. Thanks are also extended to Kate Shaw of Information Management Services (IMS) of Martin Marietta Energy Systems, Inc., for her technical review of the manuscript. Last, we wish to thank Jamie Payne, IMS Graphics Group, for her creative design of the cover of this report. We also wish to acknowledge the long-standing support of CDIAC by the U.S. Department of Energy (DOE), Global Change Research Program in the Environmental Sciences Division of DOE's Office of Health and Environmental Research.

CDIAC is located in the Environmental Sciences Division of Oak Ridge National Laboratory (ORNL), which is managed by Martin Marietta Energy Systems, Inc., for the U.S. Department of Energy under contract DE-AC05-84OR21400. 


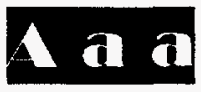

A-S
AA
AAAS

AAF

AAG

AAOE

AAS

AASE

AASP

AASSREC

AATSR

AAWORD

ABE

ABL

ABLE

ACA

ACABQ

ACARS

ACARS

ACAST

ACATs

ACC

ACC

ACC

ACCI
Arakawa-Schubert

atomic absorption

American Association for the

Advancement of Science

Association Aeronautique et

Astronautique de France

American Association of

Geographers

Airborne Antarctic Ozone

Experiment

atomic absorption spectrometry

(spectrometer, spectroscopy)

Airborne Arctic Stratospheric

Expedition

American Association of

Stratigraphic Palynologists

Association of Asian Social

Science Research Councils

advanced along-track scanning

radiometer

Association of African Women for

Research and Development

Advisory Board on Energy (India)

atmospheric boundary layer

Atmospheric Boundary Layer

Experiment

Advanced Computer Applications (project)

Advisory Committee on

Administrative and Budgetary

Questions

Aircraft Communication and

Recording System

ARINC Communications and

Reporting System

Advisory Committee on the

Application of Science and

Technology to Development (UN)

Airborne Chromatograph for

Atmospheric Trace Species

Administrative Coordination

Committee (ECOSOC)

Air Combat Command (formerly

TAC)

Antarctic Circumpolar Current

Advisory Committee on Climate

Applications and Data (WMO)
ACCM

ACCP

ACD

ACE

ACE

ACE

ACGIH

ACID

ACL

ACM

ACMAD

ACP

ACR

ACRIM

ACS

ACSM

ACSyS

ACTS

ADAAC

ADALT

ADB

ADC

ADCLS

ADDNET

ADEOS

ADEOS

ADP
Atmospheric General Circulation

Model

Atlantic Climate Change

Programme

Aeronautical Charting Division

(NOS)

Advisory Committee on the

Environment (ICSU)

Aerosol Characterization

Experiment (IGAC)

Atmospheric Chemistry Education

in Global Change (IGAC)

American Conference of

Governmental Industrial Hygienists

Acidification Chemistry

Information Data Base (BNL)

alternate concentration level

(limit?)

advanced climate model

African Centre of Meteorological

Applications for Development

Atmospheric Chemistry Program (DOE)

active cavity radiometer

active cavity radiometer irritancy monitor

American Chemical Society

American Congress of Surveying and Mapping

Arctic Climate System Study

African Centre for Technology Studies

Affiliated Distributed Active

Archive Center

Advanced Radar Altimeter

Asian Developing Bank

Affiliated Data Center

Advanced Data Collection and

Location System

Acid Deposition Data Network

(EPA)

Advanced Earth Observation

Satellite (Japan)

Advanced Earth Observing System

automatic data processing 
ADPE

$\mathrm{AE}$

AEC

AEDD

AEEI

AEEI

AER

AER

AERI

AERI

AERI-FEP

AEROCE

AES

AEST

AET

AFDM

AFGL

AFGWC

AFS

AGA

AGAL

AGASP

AGCM

AGID

AGL

AGU

Agr.

AGRHYMET

AGU

AGWAT

AIAA automatic data processing

equipment

Atmosphere Explorer

U.S. Atomic Energy Commission (now DOE)

Arctic Environment Data Directory

Atmospheric Emitted Radiance

Instrument

Autonomous energy-efficiency

index

Atmospheric and Environmental

Research, Inc. (Cambridge, Mass.)

atmospheric and environmental

research (DOE)

Atmospheric Emitted Radiance

Instrument

Atmospheric Emitted Radiance

Interferometer

AERI Front End Processor

Atmosphere/Ocean Chemistry

Experiment

Atmospheric Environment Service

(Canada)

Australian Eastern Standard Time

actual evapotranspiration

ash free dry mass

Air Force Geophysics Laboratory

Air Force Global Weather Central (U.S.)

American Fisheries Society

American Gas Association

Australian Government Analytical

Laboratories

Arctic Gas and Aerosol Sampling

Programme

Atmospheric General Circulation

Model

Association for Geoscientists for

International Development

above ground level

American Geophysical Union

Agriculture (USDA)

Agronmeteorology and Operational

Hydrology and Their Applications

American Geophysical Union

Agriculture and Water Project (JECOR)

American Institute of Aeronautics and Astronautics
AIBS

AIChE

AICRIP

AID

AIHA

AIM

AIME

AIP

AIP-1

AIP-2

AIP-3

AIREP

AIRS

AIRS-AMSU

AIS

AIT

AL

Al

ALA

ALADIN

ALARA

ALBIOS

ALE

ALE/GAGE

ALEXIS

Alf

ALFA

ALK

ALOHA

ALPEX

ALS
American Institute of Biological Sciences

American Institute of Chemical

Engineers

All India Coordinated Programme

for Rice Improvement

Agency for International

Development (U.S.)

American Industrial Hygiene

Association

approximate inertial manifold

American Institute of Mining and

Metallurgical Engineers

Algorithm Intercomparison

Programme

Algorithm Intercomparison

Project-1

Algorithm Intercomparison

Project-2

Algorithm Intercomparison

Project-3

Aircraft Weather Report

atmospheric infrared sounder

Atmospheric Infrared-Advanced

Microwave Sounding Unit

airborne imaging spectrometer

Asian Institute of Technology

Aeronomy Laboratory (Boulder, Colo.) (ERL)

aluminum

American Library Association

Atmospheric Laser Doppler

Instrument

as low as reasonably achievable

Aluminum Biogeochemistry in

Soils (EPRD)

Atmospheric Lifetime Experiment

ALE/Global Atmospheric Gases

Experiment

Array of Low-Energy X-Ray

Imaging Sensors

Grimco A-700 processor used for data analysis

AER Local Forecast and

Assimilation (Model)

alkalinity

A Long-term Oligotrophic Habitat

Assessment

Alpine Experiment

Adirondack Lake Survey (ALSC) 
GLI IL I IVEE ACRONYMS ANID ABBREVIAIIIONS

\begin{tabular}{|c|c|c|c|}
\hline ALSC & $\begin{array}{l}\text { Adirondack Lake Survey } \\
\text { Corporation } \\
\text { Aluminum in Streams Study }\end{array}$ & ANSTO & $\begin{array}{l}\text { Australian Nuclear Science and } \\
\text { Technology Organization (Menai, } \\
\text { New South Wales) }\end{array}$ \\
\hline & (EPRI) & ANT & Antarctica (START) \\
\hline ALT & altimeter & ANTARES & Antarctic research (France) \\
\hline ALTA & Active Long Term Archive & & (JGOFS) \\
\hline $\begin{array}{l}\text { AMA } \\
\text { AMAD }\end{array}$ & $\begin{array}{l}\text { African Mountains Association } \\
\text { activity median aerodynamic }\end{array}$ & ANTARFIX & $\begin{array}{l}\text { Antarctic fixed station (France) } \\
\text { (JGOFS and WOCE) }\end{array}$ \\
\hline AMAP & $\begin{array}{l}\text { diameter } \\
\text { Arctic Monitoring and Assessment } \\
\text { Programme }\end{array}$ & $\begin{array}{l}\text { ANU } \\
\text { AO }\end{array}$ & $\begin{array}{l}\text { Australian National University } \\
\text { (Canberra, Australia) } \\
\text { announcement of opportunity }\end{array}$ \\
\hline AMeDAS & $\begin{array}{l}\text { Automated Meteorological Data } \\
\text { Acquisition System }\end{array}$ & $\begin{array}{l}\text { AO } \\
\text { AODC }\end{array}$ & $\begin{array}{l}\text { atmosphere-ocean } \\
\text { acridine orange direct count }\end{array}$ \\
\hline $\begin{array}{l}\text { AMEX } \\
\text { AM/FM }\end{array}$ & $\begin{array}{l}\text { Atlantic Meteorology Experiment } \\
\text { Automated Mapping/Facilities } \\
\text { Management (Society) }\end{array}$ & $\begin{array}{l}\text { AOIPS } \\
\text { AOL }\end{array}$ & $\begin{array}{l}\text { Atmospheric and Oceanographic } \\
\text { Information Processing System } \\
\text { Airborne Oceanographic Lidar }\end{array}$ \\
\hline $\begin{array}{l}\text { AMI } \\
\text { AMIE }\end{array}$ & $\begin{array}{l}\text { active microwave instrumentation } \\
\text { Automated Methane Instrument } \\
\text { Evaluation }\end{array}$ & AOML & $\begin{array}{l}\text { Atlantic Oceanographic and } \\
\text { Meteorological Laboratory } \\
\text { (Miami) (ERL) }\end{array}$ \\
\hline AMIGO & $\begin{array}{l}\text { America's Inter-hemispheric } \\
\text { Geobiosphere Organization }\end{array}$ & $\begin{array}{l}\text { AOSB } \\
\text { AOSIS }\end{array}$ & $\begin{array}{l}\text { Arctic Ocean Sciences Board } \\
\text { Association of Small Island States }\end{array}$ \\
\hline AMIP & $\begin{array}{l}\text { Atmospheric Model } \\
\text { Intercomparison Project }\end{array}$ & $\begin{array}{l}\text { APA } \\
\text { APA }\end{array}$ & $\begin{array}{l}\text { Adirondack Park Agency } \\
\text { alkaline phosphatase activity }\end{array}$ \\
\hline $\begin{array}{l}\text { AMQUA } \\
\text { AMRIR }\end{array}$ & $\begin{array}{l}\text { American Quaternary Association } \\
\text { advanced medium resolution }\end{array}$ & APAFO & $\begin{array}{l}\text { Advanced Particles and Fields } \\
\text { Observer }\end{array}$ \\
\hline AMS & $\begin{array}{l}\text { imaging radiometer } \\
\text { accelerator mass spectrometry }\end{array}$ & APAK & $\begin{array}{l}\text { absoroed pnotosyntnencally acrive } \\
\text { radiation }\end{array}$ \\
\hline AMS & American Mathematical Society & APARE & East Asian-North Pacific Regional \\
\hline $\begin{array}{l}\text { AMS } \\
\text { AMS }\end{array}$ & $\begin{array}{l}\text { American Meteorological Society } \\
\text { Applied Mathematical Sciences } \\
\text { (DOE) }\end{array}$ & APCA & $\begin{array}{l}\text { Experiment (IGAC) } \\
\text { Air Pollution Control Association } \\
\text { (Pittsburgh) }\end{array}$ \\
\hline AMSR & $\begin{array}{l}\text { advanced microwave scanning } \\
\text { radiometer }\end{array}$ & APEC & $\begin{array}{l}\text { Asia Pacific Economic } \\
\text { Cooperation Organization }\end{array}$ \\
\hline $\begin{array}{l}\text { AMSU } \\
\text { AMSU-A }\end{array}$ & $\begin{array}{l}\text { advanced microwave sounding unit } \\
\text { advanced microwave sounding }\end{array}$ & APHA & $\begin{array}{l}\text { American Public Health } \\
\text { Association }\end{array}$ \\
\hline AMTS & $\begin{array}{l}\text { unit- } A \\
\text { advanced moisture and temperature } \\
\text { sounder }\end{array}$ & $\begin{array}{l}\text { API } \\
\text { APMP }\end{array}$ & $\begin{array}{l}\text { American Petroleum Institute } \\
\text { Acid Precipitation Mitigation } \\
\text { Program (NAPAP) }\end{array}$ \\
\hline $\begin{array}{l}\text { AMU } \\
\text { ANARE }\end{array}$ & $\begin{array}{l}\text { atomic mass unit } \\
\text { Australian National Antarctic } \\
\text { Research Establishment }\end{array}$ & $\begin{array}{l}\text { APN } \\
\text { APSRS }\end{array}$ & $\begin{array}{l}\text { Asia-Pacific Network (for Global } \\
\text { Change Research) (START) } \\
\text { Aerial Photo Summary Records }\end{array}$ \\
\hline ANBS & $\begin{array}{l}\text { Asian Network of Biological } \\
\text { Sciences }\end{array}$ & APT & $\begin{array}{l}\text { System } \\
\text { automatic picture transmission }\end{array}$ \\
\hline ANC & acid-neutralizing capacity & AQA & American Quaternary Association \\
\hline ANL & Argonne National Laboratory & ARA & atmospheric radiation analysis \\
\hline ANL/ACRF & $\begin{array}{l}\text { ANL's Advanced Computing } \\
\text { Research Facility }\end{array}$ & ARAC & $\begin{array}{l}\text { Atmospheric Release Advisory } \\
\text { Capability (program) }\end{array}$ \\
\hline ANSI & $\begin{array}{l}\text { American National Standards } \\
\text { Institute }\end{array}$ & $\begin{array}{l}\text { ARC } \\
\text { ARC }\end{array}$ & $\begin{array}{l}\text { Ames Research Center } \\
\text { Antarctic Research Center }\end{array}$ \\
\hline
\end{tabular}




\begin{tabular}{|c|c|}
\hline ARC/NNFO & GIS software \\
\hline ARCS & $\begin{array}{l}\text { Atmospheric Radiation and Cloud } \\
\text { Station }\end{array}$ \\
\hline ARCSS & Arctic System Science (program) \\
\hline ARGOS & $\begin{array}{l}\text { Argos Data Collection and Position } \\
\text { Location System (France) }\end{array}$ \\
\hline ARINC & Aeronautical Radio, Inc. \\
\hline ARINC & Aeronautical Research, Inc. \\
\hline ARISTOTELES & $\begin{array}{l}\text { Applications and Research } \\
\text { Involving Space Technologies } \\
\text { Observing the Earth's Field from a } \\
\text { Low Earth Orbiting Satellite }\end{array}$ \\
\hline ARL & Air Resources Laboratory (NOAA) \\
\hline ARL & $\begin{array}{l}\text { Australian Radiation Laboratory } \\
\text { (Melboume, Victoria) }\end{array}$ \\
\hline ARM & $\begin{array}{l}\text { Atmospheric Radiation } \\
\text { Measurement (program) (DOE) }\end{array}$ \\
\hline ARMA & Autoregressive moving-average \\
\hline ARMSAT & $\begin{array}{l}\text { Atmospheric Radiation } \\
\text { Measurement Satellite }\end{array}$ \\
\hline ARRCC & $\begin{array}{l}\text { Analysis of Rapid and Recent } \\
\text { Climate Change (project) }\end{array}$ \\
\hline ARS & $\begin{array}{l}\text { Agricultural Research Service } \\
\text { (USDA) }\end{array}$ \\
\hline ARSMC & $\begin{array}{l}\text { ASEAN Regional Specialized } \\
\text { Meteorological Centre }\end{array}$ \\
\hline ART & Arctica (START) \\
\hline ARTEMIS & $\begin{array}{l}\text { Advanced Relay and Technology } \\
\text { Mission Satellite (ESA) }\end{array}$ \\
\hline ARTEP & $\begin{array}{l}\text { Ariane Technology Experiment } \\
\text { Platform (European) }\end{array}$ \\
\hline ARW & Advanced Research Workshop \\
\hline AS & $\begin{array}{l}\text { Anti-Spoofing on GPS } \\
\text { Transmissions }\end{array}$ \\
\hline ASA & American Statistical Association \\
\hline ASA & Antarctic Support Association \\
\hline ASA & $\begin{array}{l}\text { Atmosphere Spectroscopy } \\
\text { Applications }\end{array}$ \\
\hline ASAP & $\begin{array}{l}\text { Automated Shipboard Aerological } \\
\text { Programme }\end{array}$ \\
\hline ASAR & $\begin{array}{l}\text { Advanced Synthetic Aperture } \\
\text { Radar }\end{array}$ \\
\hline ASAS & Advanced Solid State Array Sensor \\
\hline ASB & $\begin{array}{l}\text { Association of Southeastern } \\
\text { Biologists }\end{array}$ \\
\hline ASCAT & advanced scatterometer \\
\hline ASCATT & advanced scatterometer \\
\hline ASCE & $\begin{array}{l}\text { American Society of Civil } \\
\text { Engineers }\end{array}$ \\
\hline
\end{tabular}

ASCEND

ASChE

ASCII

ASCOT

ASCS

ASDAR

ASE

ASEAMS

ASEAN

ASF

ASI

ASK

ASL

ASL

ASLO

ASM

ASME

ASOEN

ASOS

ASPP

ASPRS

ASR

ASSAS

ASTER

ASTER

ASTEX

ASTM
Agenda of Science for Environment and Development into the 21st Century

American Society of Chemical Engineers

American Standard Code for Information Interchange

Atmospheric Studies in Complex Terrain (program)

Agricultural Stabilization and Conservation Service

Aircraft-to-Satellite Data Relay

Air Sea Experiment

Association of Southeast Asian

Marine Scientists

Association of Southeast Asian Nations

Alaska SAR Facility

Agenzia Spaziale Italiana (Italian Space Agency)

Assisted Search for Knowledge above sea level atmospheric surface layer American Society of Limnology and Oceanography

Applied Simulation and Modelling (IASTED)

American Society of Mechanical Engineers

ASEAN Senior Officials on the Environment

Automated Surface Observing System

American Society of Plant Physiologists

American Society for

Photogrammetry and Remote

Sensing

aerosol solar radiation

Advanced Solid State Array Sensor

Advanced Spaceborne Thermal

Emission and Reflection

(radiometer)

Atmosphere-Surface Turbulent

Exchange Research (formerly

ITIR)

Atlantic Stratocumulus Transition

Experiment

American Society for Testing and Materials (Philadelphia) 
CLCIAL G FAYE ACDONYMS AND ABBREVIATIIONS

ATES

ATDD

ATDI

ATLAS

ATLID

atm

ATM

ATMOS

ATOVS

ATP

ATPEX

ATS

ATS

ATS

ATSR

AURIO

AV

AVCS

AVD

AVHRR

AVHRR LAC AVHRR GAC AVIRIS

AVNIR

AWDB

AWIPS-90

AWRA

AWS

AWWA

AXCTD
Alcatel Espace Systems

Atmospheric Turbulence and Diffusion Division (formerly ATDL) (NOAA)

Atmospheric Turbulence and Diffusion Laboratory (now ATDD) Atmospheric Laboratory for Applications and Science atmospheric lidar atmosphere

Atmospheric Transport Model Atmospheric Trace Molecules Observed by Spectroscopy Advanced TOVS adenosine triphosphate ARM Tropical Pacific Experiment Advanced Technology Satellite Antarctic Treaty System applications technology satellite along-track scanning radiometer Auroral Imaging Observatory acoustic velocity (geophysical logging)

Advanced Vidicon Camera System acoustic variable density (geophysical logging)

advanced very high resolution radiometer (NOAA)

AVHRR Local Area Coverage AVHRR Global Area Coverage airborne visible and infrared imaging spectrometer advanced visible and near-infrared radiometer

Adirondack Watershed Data Base Advanced Weather Interactive Processing System for the $90 \mathrm{~s}$ American Water Resources Association Association for Women in Science American Water Works Association (Denver) autonomous expendable CTD profiler 


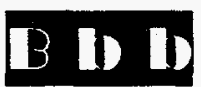

b/w

$\mathrm{BaA}$

BAAS

BAHC

BALTEX

BAO

$\mathrm{BaP}$

BaPDE

BAPMoN

BASC

BASE

BATAN

BATERISTA

BATGE

BATS

BATS

BAU

BBHIR

BBHSR

BBL

BBSR

BBSS

BBZ

BC

BCD

BCF

BEMS

BEST black and white

benz[a]anthracene

British Association for the

Advancement of Science

Biospheric Aspects of the

Hydrological Cycle (program)

(IGBP)

BAHC Scientific Steering

Committee

Baltic Sea Experiment

Boulder Atmospheric Observatory

benzo[a]pyrene

chemically reactive diolepoxides

Background Air Pollution

Monitoring Network

Board on Atmospheric Science and

Climate (U.S.)

Beaufort and Arctic Storms

Experiment

Reactor and Nuclear Research

Facilities of the National Atomic

Energy Agency

Biosphere-Atmosphere Transfers

and Ecological Research in Situ

Studies in Amazonia (BAHC and GEWEX)

Biosphere-Atmosphere Trace Gas

Exchange (in the tropics) (IGAC)

Bermuda Atlantic Time Series

Biosphere-Atmosphere Transfer

Scheme (NCAR)

business-as-usual

broadband hemispherical in flux

radiometer

broadband hemispherical solar

radiometer

benthic boundary layer

Bermuda Biological Station for

Research

balloon-borne sounding system

benthic boundary zone

black carbon

Binary Coded Decimal

bioconcentration factor

Bioelectromagnetics Society

Bilan Energetique du Systeme

Tropical
Bet

BGC

BIA

BIBEX

BIG-TT

BIH

BIOME

BIOMOVS

BIOSYNOP

BIOTRANS biological transformations (site at $47^{\circ} \mathrm{N} 20^{\circ} \mathrm{W}$

BIOTROP Regional Center for Tropical Biology

BIPM Bureau International de Poids et Mesures

BLM Bureau of Land Management (DOI)

BMAP Biological Monitoring and Abatement Program

BMC

BMFT

BMG

BMRC

BMWT

BNA

BNAP

BNL

BNSC

BOB

BOC

BOD

BODC

BOF

BOFS

BOM

BoM

BOR
Baltic Marine Cooperation Bundes Ministerium fur Forschung und Technologie (Germany)

Meteorological and Geophysics Agency

Bureau of Meteorology Research

Centre (Australia)

Biofuels and Municipal Waste

Technology (Division) (DOE)

Block Numbering Area

Basic Hydrological Network

Assessment Project

Brookhaven National Laboratory

British National Space Center

Bureau of Census (DOC)

Biochemical Oxygen Demand

British Oceanographic Data Centre (Bidston)

basic oxygen furnace

Biogeochemical Ocean Flux Study

(U.K.)

benthic organic matter

Bureau of Meteorology (DASET)

Bureau of Reclamation (DOD)
Bureau of the Budget 


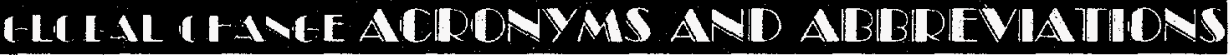

\begin{tabular}{|c|c|}
\hline BOREAS & $\begin{array}{l}\text { Boreal Ecosystems Atmosphere } \\
\text { Study (BAHC and GEWEX) }\end{array}$ \\
\hline BORIS & BOREAS Information System \\
\hline BP & before present \\
\hline BPA & Bonneville Power Administration \\
\hline BPKM & billion passenger kilo metre \\
\hline BPPT & $\begin{array}{l}\text { Agency for Assessment and } \\
\text { Application of Technology } \\
\text { (Indonesia) }\end{array}$ \\
\hline $\mathbf{B q}$ & $\begin{array}{l}\text { Becquerel (symbol) (SI unit of } \\
\text { activity of ionizing radiation } \\
\text { source) }\end{array}$ \\
\hline BRDF & $\begin{array}{l}\text { bidirectional reflectance } \\
\text { distribution function }\end{array}$ \\
\hline $\mathrm{BrO}$ & bromine monoxide \\
\hline BRW & $\begin{array}{l}\text { Barrow Observatory (Barrow, } \\
\text { Alaska) (CMDL) }\end{array}$ \\
\hline BSIP & $\begin{array}{l}\text { Birban Sahani Institute of } \\
\text { Paleobotany }\end{array}$ \\
\hline BSRN & $\begin{array}{l}\text { Baseline Surface Radiation } \\
\text { Network }\end{array}$ \\
\hline BTCs & $\begin{array}{l}\text { breakthrough curves (column- } \\
\text { elution tests) }\end{array}$ \\
\hline BUFR & $\begin{array}{l}\text { Binary Universal Form for the } \\
\text { Representation (of data) }\end{array}$ \\
\hline BuREC & Bureau of Reclamation (now WPR) \\
\hline BUV & Backscatter Ultraviolet Radiometer \\
\hline BVE & Barotropic vorticity equation \\
\hline BW & bulk water \\
\hline BWTP & Basalt Waste Isolation Project \\
\hline
\end{tabular}




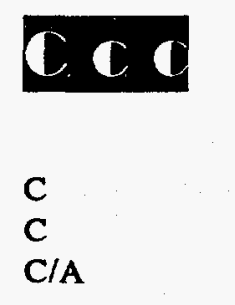

C-MACCS

C3AT

CAA

CAA

CAAP

CAC

CAC

CACGP

CAD

CADD

CAEM

CAF

CAgM

Calval

CAM

CAMS

CAO

CAP

CAPPI

\section{CAR}

CARBICE

CARDS

CARS

CARS

CART

CAS

CAS

CAS carbon

Celsius, centigrade

Coarse/Acquisition GPS signal structure

Centre for Mathematical Modeling and Computer Simulation

Ch3 Albedo Test (AVHRR)

Central Arid Asia (START)

Clean Air Act (1970)

Composition and Acidity of

Southeast Asian Precipitation

Climate Analysis Centre (now

CPC) (WMO)

Committee on Atmospheric

Chemistry

Commission on Atmospheric

Chemistry and Global Pollution

(IAMAS)

computer-aided design/

drafting/drawing

computer-aided design and drafting

Commission for Aeronautical

Meteorology (WMO)

Clean Air Facility

Commission for Agricultural

Meteorology (WMO)

calibration/validation

crassulacean acid metabolism

Control and Monitoring System

cold air outbreak

Central Arizona Project (USBR)

Constant Altitude Plan Position

Indicator

Caribbean (START)

Carbon Dioxide Intercalibration

Experiment (IGAC)

climatological aerological

reference data set

Climate Applications Referral

System

coherent anti-Stokes Raman

scattering

Cloud and Radiation Testbed

(ARM)

Chemical Abstract Series

Chinese Academy of Science

Commission for Atmospheric

Sciences (WMO)
CASA Inter-Union Commission on the Application of Science to Agriculture, Forestry, and Aquaculture (ICSU)

CASSAD Centre for African Settlement Studies and Development

CAST

CAT

CBA

CBO

CBS

CCAM

CCAQ

$\mathrm{CCCN}$

$\mathrm{CCCO}$

CCCP

CCD

CCDP

$\mathrm{CCI}$

CCIR

Chinese Academy of Science and

Technology

computerized axial tomography

cost benefit analysis

Congressional Budget Office

(U.S.)

Commission for Basic Systems

(WMO)

Chikuko Cha Amayi M'Malawi

(Women's NGO, Malawi)

Consultative Committee on

Administrative Questions

CDIAC Computing System

Network

Committee on Climate Changes and the Ocean (SCOR and IOC)

California Institute of Technology

Concurrent Computation Project

charge-coupled device (camera)

Climate Change Detection Project

Commission for Climatology

(WMO)

International Radio Consultative

Committee (ITU)

CCITT International Consultative

Committee on Telegraphs and

Telecommunications (ITU)

CCIW

$\mathrm{CCM}_{1}$

$\mathrm{CCM}_{2}$

CCM

CCMO

Canadian Centre for Inland Waters

community climate model one

community climate model two

(NCAR)

community climate model

Community Climate Model

(National Center for Atmospheric

Research)

CCM2 Community Climate Model

(version 2)

CCMS Committee on the Challenges of

Modern Society

CCN cloud condensation nucleus 
HLIL IFAYE ACRONYMS ANID ABBREVIATIIONS

CCOP

CCSDS

CCT

CD

$\mathrm{CD}$

CD

CDAS

CDC

CDCR

CDDIS

CDF

CDIAC

CDIC

CDMS

CDOS

CDP

CDRD

CD-ROM

CDRP

CE

CEA

CEA

CEC

CEC

CEDAR

CEDDA

CEEM

CEES
Committee for the Coordination of Joint Prospecting for Mineral

Resources in Asian Offshore Areas (Indonesia)

Canadian Centre for Remote

Sensing (Canada)

Consultative Committee for

Standard Data Services

computer-compatible tape

Chemistry Division

community development

compact disc

Climate Data Assimilation System

Control Data Corporation

conceptual design and cost review

Crustal Dynamics Data

Information System

common data format

Carbon Dioxide Information

Analysis Center (ORNL/DOE)

(formerly CDIC)

Carbon Dioxide Information

Center (now CDIAC)

Cryospheric Data Management

System

Customer Data and Operations

System

Crustal Dynamics Project (NASA)

Carbon Dioxide Research Division compact disc-read only memory

Carbon Dioxide Research Program

Office of Conservation and

Renewable Energy

cause-and-effect analysis

Central Electricity Authority

(India)

cation exchange capacity

Commission of European

Communities

Coupled Energetics and Dynamics

of the Atmospheric Regions

Center for Experiment Design and

Data Analysis (NOAA)

Center for Energy and

Environmental Management

Committee on Earth and

Environmental Sciences (formerly

CES; replaced by CENR)
CEI

CEM

CEMA

CEN

CENELEC

CENR

CENTO

CEOS

CEP

CEP

CEPAL

CEPAL

CEPEX

CEPT

CEQ

CER

CERCLA

CERES

CERES

CERGA

CERL

CERN

CERN
Center for Environmental Information

cumulus ensemble model

Council of Mutual Economic Aid

(Russian Federation, formerly

U.S.S.R.)

European Committee for

Standardization (EC)

European Committee for

Electrotechnical Standardization

(EC)

Committee on Environmental and Natural Resources (U.S. NCS\&T)

Central Treaty Organization

Committee on Earth Observation

Satellites

circular error probability

Comite Ecumenico de Proyectos

Comision Economica para America

Latina (Spain) (ECLA)

Economic Commission for Latin

America (Spain) (ECLA)

Central Equatorial Pacific

Experiment

Conference Europeenne des

Administration des Postes et

Telecommunications (European

Conference of Postal and

Telecommunications

Administrations)

Council on Environmental Quality (U.S.)

carbon dioxide exchange rate

Comprehensive Environmental

Response, Compensation, and

Liability Act (1980) (Superfund)

Clouds and the Earth's Radiant

Energy System (EOS)

Crop Environment Resource Synthesis

Centre d'Etudes et de Rechereches

Geodynamiques et Astronomiques

Corvallis Environmental Research

Laboratory (EPA)

Chinese Ecological Research

Network

European Laboratory for Particle

Physics 
TH IL IFAYE ACRONYMS AND ABBREVIIATIIDNS

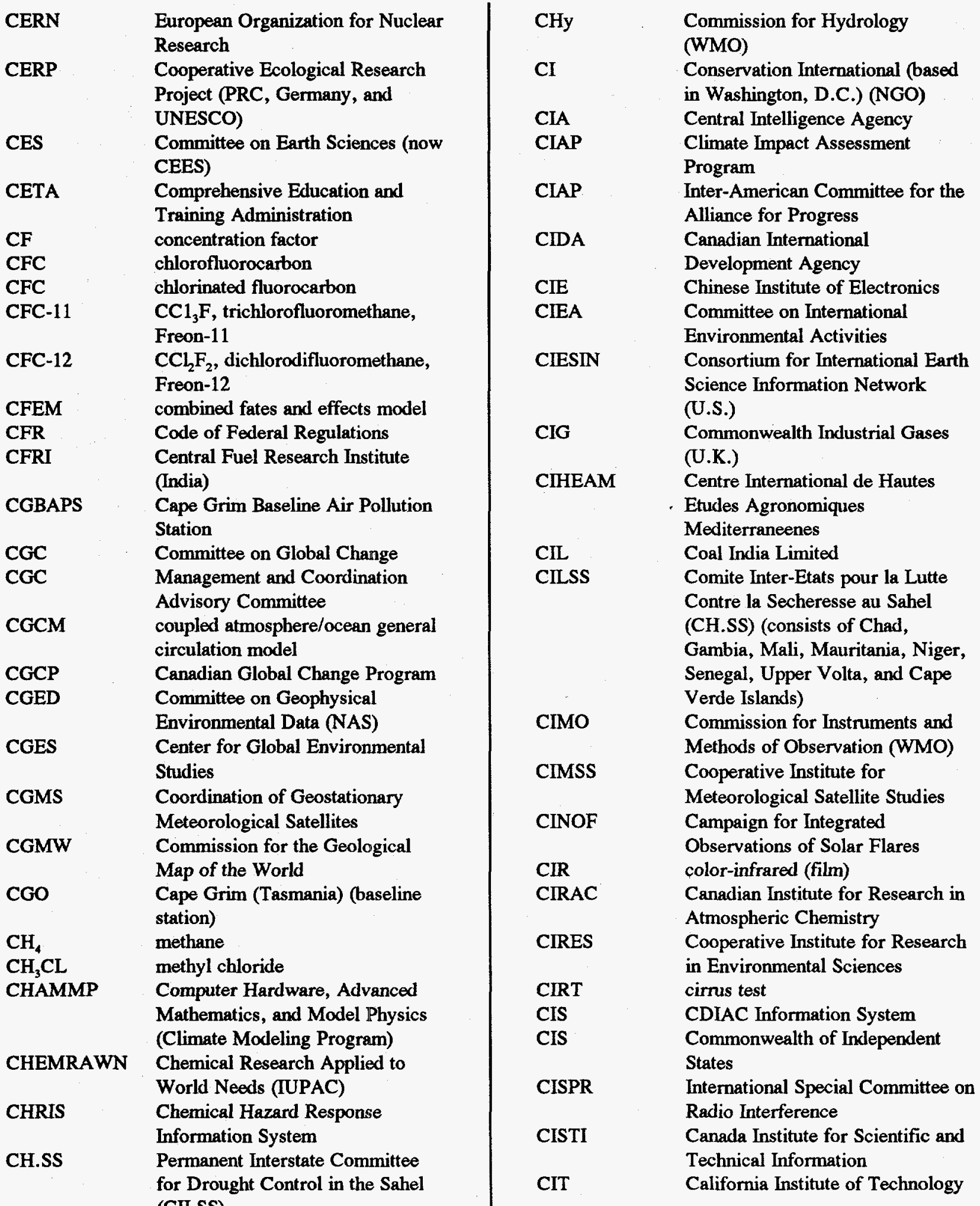
(CILSS) 
GLI-IL C FAVE ACDONYMS AND ABBDEVIATIIONS

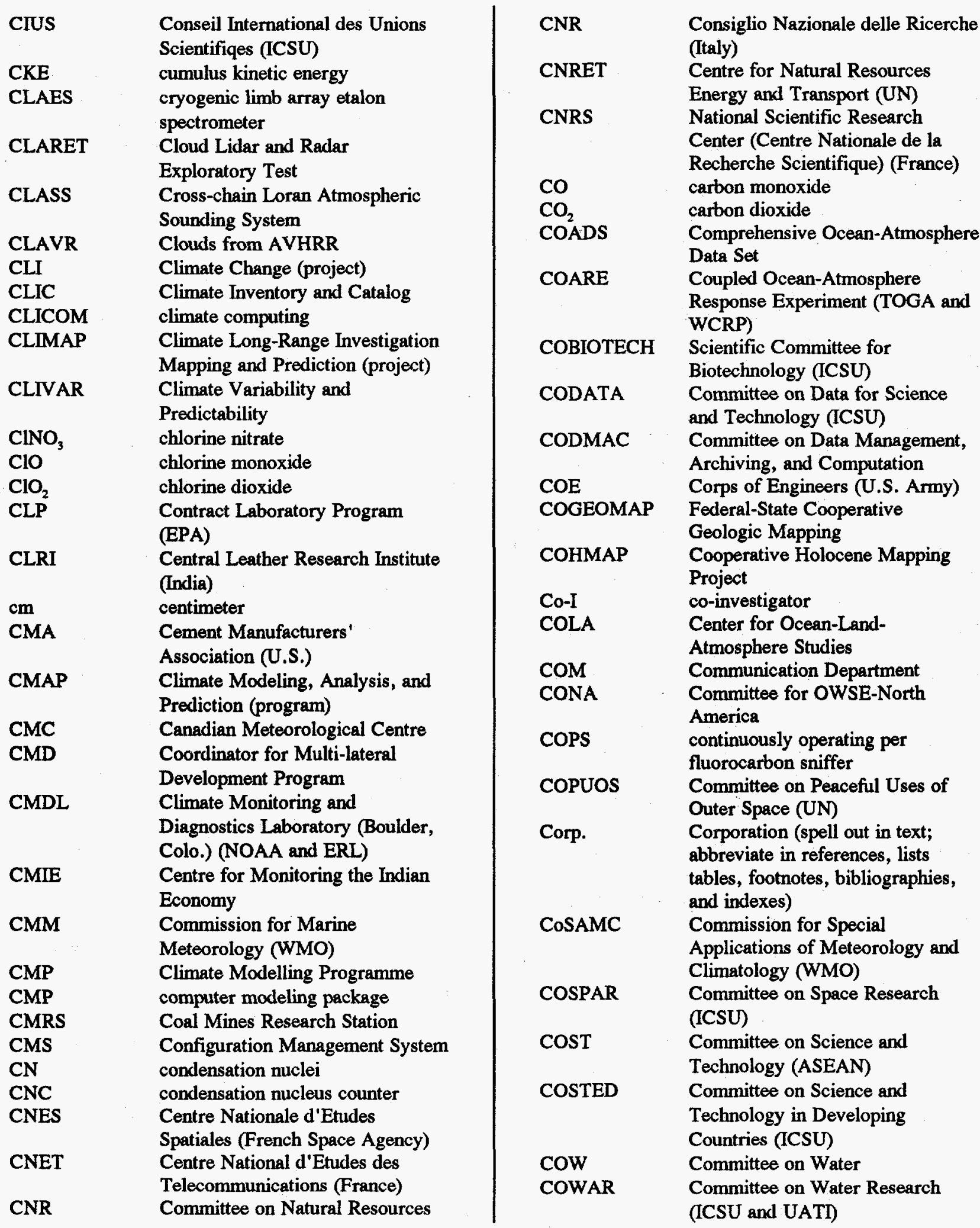




\section{GLI IL I FATE ACDONYMS AND AVBBREVIATIIONS}

\begin{tabular}{|c|c|c|c|}
\hline COWRR & $\begin{array}{l}\text { Committee on Water Resources } \\
\text { Research }\end{array}$ & CSIR & $\begin{array}{l}\text { Council of Scientific and Industrial } \\
\text { Research (India) }\end{array}$ \\
\hline CPC & $\begin{array}{l}\text { Climate Prediction Center } \\
\text { (formerly CAC) }\end{array}$ & CSIRO & $\begin{array}{l}\text { Commonwealth Scientific and } \\
\text { Industrial Research Organization }\end{array}$ \\
\hline CPM & critical path method & & (Australia) \\
\hline CPMS & $\begin{array}{l}\text { climatological prediction by model } \\
\text { statistics }\end{array}$ & CSIRO/DAR & $\begin{array}{l}\text { CSIRO/Division of Atmospheric } \\
\text { Research (Australia) }\end{array}$ \\
\hline CPO & Core Project Office (IGBP) & CSM & climate system monitoring \\
\hline CPP & cloud photopolarimeter & CSMP & Climate System Modeling Program \\
\hline CPPC & $\begin{array}{l}\text { Core Project Planning Committee } \\
\text { (IGBP) }\end{array}$ & $\begin{array}{l}\text { CSO } \\
\text { CSPP }\end{array}$ & $\begin{array}{l}\text { Central Statistical Organization } \\
\text { Collaborative Special Project and }\end{array}$ \\
\hline CPRS & cloud-profiling radar system & & Programme \\
\hline CPS & certified professional secretary & CSR & Centra de Sensores Remotes (Italy) \\
\hline $\begin{array}{l}\text { CPU } \\
\text { CPUE }\end{array}$ & $\begin{array}{l}\text { central processing unit } \\
\text { catch per unit effort }\end{array}$ & CSRS & $\begin{array}{l}\text { Cooperative State Research } \\
\text { Service }\end{array}$ \\
\hline CRC & Climate Research Committee & CST & CHAMMP Science Team \\
\hline CREN & $\begin{array}{l}\text { Chemical Research and } \\
\text { Environmental Needs }\end{array}$ & $\begin{array}{l}\text { CSU } \\
\text { CTD }\end{array}$ & $\begin{array}{l}\text { Colorado State University } \\
\text { Chemical Technology Division }\end{array}$ \\
\hline CREO & $\begin{array}{l}\text { Cryosphere Research on Qingzang } \\
\text { Plateau }\end{array}$ & CTD & $\begin{array}{l}\text { conductivity, temperature, and } \\
\text { depth sensor }\end{array}$ \\
\hline CRESP & $\begin{array}{l}\text { Coordinated Research and } \\
\text { Environmental Surveillance } \\
\text { Programme (NEA) }\end{array}$ & $\begin{array}{l}\text { CTD } \\
\text { CTS }\end{array}$ & $\begin{array}{l}\text { continuous-temperature-depth } \\
\text { Committee on the Teaching of } \\
\text { Science }\end{array}$ \\
\hline CRF & cloud radiative forcing & CTSS & Cray time-sharing system \\
\hline CRI & $\begin{array}{l}\text { Climate Research Institute } \\
\text { (Oklahoma State University) }\end{array}$ & $\begin{array}{l}\text { CUF } \\
\text { C.V. }\end{array}$ & $\begin{array}{l}\text { capacity utilization factor } \\
\text { coefficient of variation (math) }\end{array}$ \\
\hline CRIC & $\begin{array}{l}\text { Computerized River Information } \\
\text { System (U.S. Fish and Wildlife } \\
\text { Service) }\end{array}$ & $\begin{array}{l}\text { CVS } \\
\text { CW } \\
\text { CWA }\end{array}$ & $\begin{array}{l}\text { Conference and Visitors' Services } \\
\text { continuous wave } \\
\text { Clean Water Act (1977) }\end{array}$ \\
\hline CRM & certified reference material & CWR & continuous-wave radar \\
\hline CRP & Climate Research Program & CWSF & Chemical Waste Storage Facility \\
\hline CRPE & $\begin{array}{l}\text { Centre de Recherches en Physique } \\
\text { de l'Environment Terrestre et } \\
\text { Planetaria }\end{array}$ & $\begin{array}{l}\text { CZCS } \\
\text { CZM } \\
\text { CZMA }\end{array}$ & $\begin{array}{l}\text { coastal zone color scanner } \\
\text { Coastal Zone Management } \\
\text { Coastal Zone Management Act }\end{array}$ \\
\hline CRREL & $\begin{array}{l}\text { Cold Regions Research and } \\
\text { Engineering Laboratory (COE) }\end{array}$ & & (1972) \\
\hline CRS & cavity-ringdown spectrometer & & \\
\hline CRT & cathode-ray tube & & \\
\hline CRYSYS & Cryospheric System & & \\
\hline CS & Computer Services Department & & \\
\hline CSA & Canadian Space Agency & & \\
\hline CSA & Chinese Society of Astronautics & & \\
\hline CSATA & $\begin{array}{l}\text { Centra Studi e Applicazioni in } \\
\text { Tecnologie Avanzate (Italy) }\end{array}$ & & \\
\hline CSD & $\begin{array}{l}\text { Commission on Sustainable } \\
\text { Development }\end{array}$ & & \\
\hline CSELI & $\begin{array}{l}\text { Centra Studi e Laboratori } \\
\text { Telecommunicazioni (Italy) }\end{array}$ & & \\
\hline CSES & $\begin{array}{l}\text { Center for the Study of Earth from } \\
\text { Space (UC) }\end{array}$ & & \\
\hline
\end{tabular}




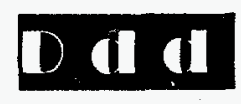

D/H
1-D
2-D
3-D
4-D
4DDA

deuterium/hydrogen (ratio)

one-dimensional

two-dimensional

three-dimensional

four-dimensional

four-dimensional (4D) data

assimilation

1-D RCM

2-D RCAM

DAAC

DAAC

DADS

DAR

DARA

DARE

DARPA

DAS

DAS

DASA

DASET

DASETT

DAT

DB

DB

dbh

DBMS

DBS

DC one-dimensional radiative-

convective model

two-dimensional radiative-

advective-convective equilibrium

model

Data Active Archive Center

Distributed Active Archive Center

Data Archive and Distribution

System

Division of Atmospheric Research (CSIRO) (Aspendale, Victoria, Australia)

Deutsche Agentur fur

Raumfahrtangelegenheiten

(Germany)

data rescue

U.S. Defense Advanced Research

Projects Agency

data-assimilation system

direct access system

Deutsche Aerospace

Administration

Department of the Arts, Sports, the Environment, Tourism, and Territories (formerly DASETT)

Department of the Arts, Sports, the Environment, Tourism, and

Territories (now DASET)

Department of Science and

Technology

direct-beam (irritancy)

direct broadcast

diameter at breast height

data base management systems

(Deutsche Forschungs-und

Versuchsanstalt fur Luft-und

Raumfahrt (Germany)

Doppler beam swinging

Department of Commerce (U.S.)
DC

D.C.

DCAR

DCDS

DCDSTF

DCP

DCP-OES

DCS

DDF

DDL

DDMP

D.DRP

DDT

DE

DEBITS

DEC

DECNet

$\mathrm{dE} / \mathrm{dt}$

DEIS

DEM

DEMR

DEW

DGCM

DGLR

DGVM

DH

DHCN

DHHS

DHS

DI developing country

District of Columbia

Danish Centre for Atmospheric

Research

Digital Cartographic Data Standard

Digital Cartographic Data

Standards Task Force (Composed

of members of the NCDCDS and

FICCDC/SWG)

data collection platform

direct coupled plasma-optical

emission spectroscopy

data collection system

descriptive data file

direct downlink

domain-decomposition-message-

passing

Direct/Delayed Response Project

dichlorodiphenyl-trichloroethane

(insecticide)

dynamics explorer

Deposition of Biogeochemically Important Trace Species (IGAC)

Digital Equipment Corporation

Digital Equipment Corporation

Network

rate of evaporation (moisture loss

from soil)

Draft Environmental Impact

Statement

digital elevation model

Department of Energy, Mines, and

Resources (Canada)

distant early warning

Dynamic Global Vegetation Model

Deutsche Gesellschaft fur Luft-und

Raumfahrt (Germany)

Dynamic Global Vegetation Model

(GAIM)

chromosome-doubled haploid

Daily Historical Climate Network

U.S. Department of Health and

Human Services

Department of Human Services

Directorate 


\begin{tabular}{|c|c|c|c|}
\hline DIA & $\begin{array}{l}\text { Denmark's Academy for } \\
\text { Engineering }\end{array}$ & $\begin{array}{l}\text { DOJ } \\
\text { DOL }\end{array}$ & $\begin{array}{l}\text { U.S. Department of Justice } \\
\text { U.S. Department of Labor }\end{array}$ \\
\hline DIAL & Differential Absorption Lidar & DOLY & Dynamic Global Phytogeography \\
\hline DIC & dissolved inorganic carbon & & Model (GCTE) \\
\hline DIDAC & digital data acquisition system & DOM & dissolved organic macromolecules \\
\hline DIF & Data Interchange Format & DOM & dissolved organic matter \\
\hline DIS & $\begin{array}{l}\text { Data and Information Systems } \\
\text { (EOS and IGBP) }\end{array}$ & $\begin{array}{l}\text { DOMSAT } \\
\text { DOMSTAR }\end{array}$ & $\begin{array}{l}\text { Domestic Communications Satellite } \\
\text { communications relay set }\end{array}$ \\
\hline DISORT & discrete ordinate (model) & DOPLID & Doppler lidar \\
\hline DIS-SC & DIS Standing Committee & DORIS & Determination d'Orbite et Radio \\
\hline DISSPLA & $\begin{array}{l}\text { Display Integrated Software } \\
\text { System and Plotting Language }\end{array}$ & & $\begin{array}{l}\text { Positionement Integre par Satellite } \\
\text { (France) }\end{array}$ \\
\hline DIW & Department of Industrial Works & DORIS & Doppler Orbitography and Radio \\
\hline DKD & Deutscher Kalibrierdienst & & Positioning Integrated by Satellites \\
\hline DLG & digital line graph & DOS & Department of Space \\
\hline DLG-E & digital line graph-enhanced & DOS & U.S. Department of State \\
\hline DLR & $\begin{array}{l}\text { Deutsche Forschungsanstalt fur } \\
\text { Luft-und Raumfahrt (Germany) }\end{array}$ & DOST & $\begin{array}{l}\text { Department of Science and } \\
\text { Technology }\end{array}$ \\
\hline DLS & dynamic limb sounder & DOT & U.S. Department of Transportation \\
\hline DM & $\begin{array}{l}\text { Data Management (WMO and } \\
\text { WWW) }\end{array}$ & $\begin{array}{l}\text { DOY } \\
\text { DP }\end{array}$ & $\begin{array}{l}\text { day of year } \\
\text { direct playback }\end{array}$ \\
\hline DM & deep monitoring (wells) & DPC & display code (CDC) \\
\hline DMA & Defense Mapping Agency (U.S.) & DPR & development planning review \\
\hline DMA & $\begin{array}{l}\text { direct memory access (computer } \\
\text { term) }\end{array}$ & $\begin{array}{l}\text { (D)PR } \\
\text { DPS }\end{array}$ & $\begin{array}{l}\text { dual precipitation radar } \\
\text { Digital Photogrammetry System }\end{array}$ \\
\hline DMDS & dimethyl disulfide & DQO & Data Quality Objectives \\
\hline DM-MIMD & $\begin{array}{l}\text { distributed memory, multiple } \\
\text { instruction, multiple data }\end{array}$ & DRAW & $\begin{array}{l}\text { Direct Read After Write Animation } \\
\text { System }\end{array}$ \\
\hline & (computer) & DRS & Data Relay Satellite \\
\hline DMS & data management system & DRSS & Data Relay Satellite System \\
\hline DMS & $\begin{array}{l}\text { dimethyl sulfide or } \\
\text { dimethylsulphide }\end{array}$ & $\begin{array}{l}\text { DSB } \\
\text { DSDP }\end{array}$ & $\begin{array}{l}\text { direct sounding broadcast } \\
\text { deep sea drilling project }\end{array}$ \\
\hline DMSO & dimethyl sulfoxide & DSIR & Department of Scientific and \\
\hline DMSP & $\begin{array}{l}\text { Defense Meteorological Satellite } \\
\text { Program (U.S.) }\end{array}$ & & $\begin{array}{l}\text { Industrial Research (now SRC) } \\
\text { (U.K.) }\end{array}$ \\
\hline DNA & deoxyribonucleic acid & DSM & demand-side management \\
\hline DND & $\begin{array}{l}\text { Department of National Defense } \\
\text { (Canada) }\end{array}$ & $\begin{array}{l}\text { DSN } \\
\text { DSP }\end{array}$ & $\begin{array}{l}\text { Deep Space Network } \\
\text { digital signal processing }\end{array}$ \\
\hline DNES & $\begin{array}{l}\text { Department of Non-conventional } \\
\text { Energy Sources (India) }\end{array}$ & $\begin{array}{l}\text { DSS } \\
\text { DST }\end{array}$ & $\begin{array}{l}\text { Data Support Section (NCAR) } \\
\text { data system tests }\end{array}$ \\
\hline DOA & U.S. Department of Agriculture & DST & Department of Science and \\
\hline DOAS & $\begin{array}{l}\text { differential optical absorption } \\
\text { spectroscopy }\end{array}$ & DTD & $\begin{array}{l}\text { Technology } \\
\text { digital terrain data }\end{array}$ \\
\hline DOC & dissolved organic carbon & DTH & Technical University of Denmark \\
\hline DOC & U.S. Department of Commerce & DU & Dobson units \\
\hline DOD & U.S. Department of Defense & DUNDEE & Down Under Doppler and \\
\hline DOE & $\begin{array}{l}\text { Department of the Environment } \\
\text { (Canada) }\end{array}$ & DWBM & $\begin{array}{l}\text { Electricity Experiment } \\
\text { Office of Defense Waste }\end{array}$ \\
\hline DOE & U.S. Department of Energy & & Byproducts Management \\
\hline DOI & U.S. Department of Interior & DWS & Doppler wind sensor \\
\hline
\end{tabular}




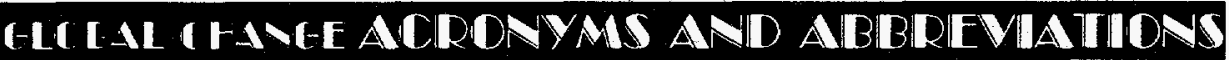

DYFAMED atmospheric dynamics and fluxes in the Mediterranean Sea (France) (JGOFS)

DYN

Dynamic Systems Project 
HLILIL IIVE ACDONYMS ANID ABBREVIATIDNS

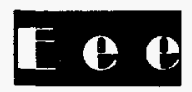

\section{EADS}

EARSeL

EAS

EAs

EASIZ

EAWEP

EBBR

EBCDIC

EBM

EC

EC

EC

EC

EC

EC

EC

ECA

ECAFE

ECAMP

ECC

ECD

ECD

ECDIS

ECE

ECG

EC-GC

ECHIVAL European International Project on Climate and Hydrological

Interactions between Vegetation, Atmosphere, and Land Surfaces (BAHC and EPOCH)
Environmental Assessment Data System

European Association of Remote

Sensing Laboratories

Environmental Analyses Section

(ORNL)

Environmental Assessments

Ecology of the Antarctic Sea Ice

Zone

East Asia and Western Pacific

Energy-Balance Bowen Ratio

Extended Binary Coded Data

Interchange Code (IBM)

energy-balance model

eddy correlation

elemental carbon

Environment Canada

European Community

European Communities or

Commission of the European

Communities

executive committee

Executive Council (WMO)

Economic Commission for Africa

(UN)

Economic Commission for Asia

and the Far East

Environmental Compliance

Assessment and Management

Program (USAF)

electrochemical concentration cell

effective cutoff diameter

electron capture detector (EC)

Electronic Chart Display and

Information Systems

Economic Commission for Europe (UN)

Ecosystem Conservation Group

(UNEP, FAO, UNESCO, and

IUCN)

electron capture-gas

chromatograph
ECLA Economic Commission for Latin

America

ECLAC Economic Commission for Latin America and the Caribbean (UN)

ECLIPS Experimental Cloud Lidar Pilot Study

ECMWF European Centre for MediumRange Weather Forecasts

Ecom.

ECOMARGE ecology of the continental margins (France) (JGOFS)

ECOSOC Economic and Social Council (UN)

ECOWARM European Committee for Water

Resources Management (IAHS)

ECS Environmentally Compatible

Energy Strategies (project)

ECS EOSDIS Core System

ECSL European Centre for Space Law

ECWA Economic Commission for

Western Asia

ED Energy Division

EDA elevation difference accuracy

EDAS particulate/radon data system

EDC EROS Data Center

EDD Exchange of Digital Data

EDI electronic Data Interchange

EDI Equivalent-damage index

EDIS Environmental Data and

Information Service

EDOS EOS Data and Operations System

EDP electronic data processing

EDS

EDSG

EDTA

EDTA

EDX

EDXS

EEC

EEI

EER

EFEDTA

e.g.
Environmental Data Service ethylenediaminetetraacetate ethylenediaminetetraacetic (acid) energy-dispersive $X$-ray (analysis) energy-dispersive $X$-ray spectroscopy

European Economic Communities Edison Electric Institute external engineering review European Field Experiment in Decertification Threatened Area (ECHIVAL and BAHC)

exempli gratia (for example) use only in parentheses or footnotes, followed by a comma)
Energy Demand Steering Group 
GII IL I FAVTE ACDONYMS ANID AMBRIEVIAIIIONS

\begin{tabular}{|c|c|c|c|}
\hline EG\&G & $\begin{array}{l}\text { Edgerton, Germeshauser, and } \\
\text { Greer }\end{array}$ & $\begin{array}{l}\text { EOF } \\
\text { EO-ICWG }\end{array}$ & $\begin{array}{l}\text { empirical orthogonal function } \\
\text { Earth Observations International }\end{array}$ \\
\hline EGS & European Geophysical Society & & Coordination Working Group \\
\hline EIA & $\begin{array}{l}\text { Energy Information Administration } \\
\text { (DOE) }\end{array}$ & $\begin{array}{l}\text { EOPM } \\
\text { EOS }\end{array}$ & $\begin{array}{l}\text { electro-optic phase modulation } \\
\text { Earth Observing System (U.S.) }\end{array}$ \\
\hline EIPO & $\begin{array}{l}\text { European IGAC Project Office } \\
\text { Steering Committee }\end{array}$ & $\begin{array}{l}\text { EOS-AERO } \\
\text { EOS-ALT }\end{array}$ & $\begin{array}{l}\text { EOS Aerosol Mission } \\
\text { EOS Altimetry Mission }\end{array}$ \\
\hline $\begin{array}{l}\text { EIS } \\
\text { EISCAT }\end{array}$ & $\begin{array}{l}\text { environmental impact statement } \\
\text { European Incoherent Scatter } \\
\text { Facility }\end{array}$ & EOS-AM & $\begin{array}{l}\text { EOS Morning Crossing } \\
\text { (ascending) Mission or Earth } \\
\text { Observing System (morning pass) }\end{array}$ \\
\hline E-LIDAR & $\begin{array}{l}\text { Ecole Inter-Etats des Sciences et de } \\
\text { Medecine Veterinaire (Dakar) } \\
\text { experimental lidar }\end{array}$ & EOSAT & $\begin{array}{l}\text { Earth Observation Satellite } \\
\text { (commercial operator for satellite } \\
\text { systems in the U.S.) }\end{array}$ \\
\hline ELS & Eastern Lake Survey & EOS-CHEM & EOS Chemistry Mission \\
\hline ELV & expendable launch vehicle & EOSDIS & EOS Data and Information System \\
\hline EM & electromagnetic & EOSP & Earth Observing Scanning \\
\hline EMAP & $\begin{array}{l}\text { Environmental Monitoring and } \\
\text { Assessment Program }\end{array}$ & & $\begin{array}{l}\text { Polarimeter } \\
\text { Earth Observing System (afternoon }\end{array}$ \\
\hline EMEP & $\begin{array}{l}\text { Cooperative Programme for } \\
\text { Monitoring and Evaluation of } \\
\text { Long-Range Transmission of Air } \\
\text { Pollutants in Europe }\end{array}$ & EOTC & $\begin{array}{l}\text { pass) or EOS Afternoon Crossing } \\
\text { (descending) Mission } \\
\text { European Organization for Testing } \\
\text { and Certification (EC) }\end{array}$ \\
\hline EMEX & Equatorial Mesoscale Experiment & EP & Earth Probe \\
\hline EML & $\begin{array}{l}\text { Environmental Measurements } \\
\text { Laboratory }\end{array}$ & EP & $\begin{array}{l}\text { extraction procedure (RCRA and } \\
\text { EPA) }\end{array}$ \\
\hline EMOC & $\begin{array}{l}\text { EOSDIS Mission Operations } \\
\text { Center }\end{array}$ & EPA & $\begin{array}{l}\text { Environmental Protection Agency } \\
\text { (USDI) }\end{array}$ \\
\hline EMR & electromagnetic radiation & EPA & Environmental Protection \\
\hline EMSL & $\begin{array}{l}\text { Environmental Monitoring Systems } \\
\text { Laboratory (EPA) }\end{array}$ & & $\begin{array}{l}\text { Authority (Victoria, Australia) } \\
\text { European Pollen Database }\end{array}$ \\
\hline EMSL-LV & $\begin{array}{l}\text { Environmental Monitoring Systems } \\
\text { Laboratory - Las Vegas (Nevada) } \\
\text { (EPA) }\end{array}$ & $\begin{array}{l}\text { EPER } \\
\text { EPI }\end{array}$ & $\begin{array}{l}\text { Environmental Processes and } \\
\text { Effects Research (program) } \\
\text { environmental productivity index }\end{array}$ \\
\hline ENRIC & $\begin{array}{l}\text { Environment and Natural } \\
\text { Resources Information Center }\end{array}$ & EPICA & $\begin{array}{l}\text { European Programme for Ice } \\
\text { Coring in Antarctica (PAGES) }\end{array}$ \\
\hline ENRICH & $\begin{array}{l}\text { European Network for Research on } \\
\text { Global Change (CEC) }\end{array}$ & EPOCH & $\begin{array}{l}\text { European Programme on Climate } \\
\text { and Hazards (EEC) }\end{array}$ \\
\hline ENSO & $\begin{array}{l}\text { El Nino/southern oscillation } \\
\text { (coupled oceanic-atmospheric } \\
\text { change) }\end{array}$ & $\begin{array}{l}\text { EPOP } \\
\text { EPOPE }\end{array}$ & $\begin{array}{l}\text { European Polar-Orbiting Platform } \\
\text { Etude de Processes dans l'Ocean } \\
\text { Pacifique Equatorial }\end{array}$ \\
\hline $\begin{array}{l}\text { ENVISAT } \\
\text { EOC }\end{array}$ & $\begin{array}{l}\text { environmental satellite } \\
\text { Earth Observation Center }\end{array}$ & EPRI & $\begin{array}{l}\text { Electric Power Research Institute } \\
\text { (Palo Alto, Calif.) }\end{array}$ \\
\hline & (NASDA) & EQPAC & Equatorial Pacific (JGOFS) \\
\hline EOC & EOS Operations Center (NASA) & ER & Office of Energy Research \\
\hline EOCAP & $\begin{array}{l}\text { Earth Observation } \\
\text { Commercialization Application } \\
\text { Program }\end{array}$ & $\begin{array}{l}\text { ER-2 } \\
\text { ERB } \\
\text { ERBE }\end{array}$ & $\begin{array}{l}\text { Extended Range U-2 (aircraft) } \\
\text { Earth Radiation Budget } \\
\text { Earth Radiation Budget Exneriment }\end{array}$ \\
\hline EODC & $\begin{array}{l}\text { Earth Observing Data Center } \\
\text { (U.K.) }\end{array}$ & ERBES & $\begin{array}{l}\text { Earth Radiation Budget Explorer } \\
\text { Satellite }\end{array}$ \\
\hline
\end{tabular}


ELIII I IVEE ACRONYMS AND AIBBREVIATIIONS

\begin{tabular}{|c|c|c|c|}
\hline \multirow{3}{*}{$\begin{array}{l}\text { ERBS } \\
\text { ERD } \\
\text { ERDA }\end{array}$} & $\begin{array}{l}\text { Earth Radiation Budget Satellite } \\
\text { Ecological Research-Division }\end{array}$ & \multirow{2}{*}{$\begin{array}{l}\text { ESAM } \\
\text { ESCAP }\end{array}$} & \multirow{3}{*}{$\begin{array}{l}\text { Institute for Environmental Science } \\
\text { and Management } \\
\text { Economic and Social Commission } \\
\text { for Asia and the Pacific (UN) } \\
\text { Environmental Sciences Division } \\
\text { (ORNL) }\end{array}$} \\
\hline & (DOE and OHER) & & \\
\hline & $\begin{array}{l}\text { U.S. Energy Research and } \\
\text { Development Administration } \\
\text { (formerly AEC; now DOE) }\end{array}$ & ESD & \\
\hline ERDAS & $\begin{array}{l}\text { Earth Resources Data Analysis } \\
\text { Systems }\end{array}$ & ESDIS & $\begin{array}{l}\text { Earth Science Data and } \\
\text { Information System }\end{array}$ \\
\hline ERFU & $\begin{array}{l}\text { Environmental Restoration and } \\
\text { Facilities Upgrade Program } \\
\text { Environmental Research Institute }\end{array}$ & $\begin{array}{l}\text { ESF } \\
\text { ESF/EPC }\end{array}$ & $\begin{array}{l}\text { European Science Foundation } \\
\text { ESF/European Palaeoclimate and } \\
\text { Man Project (holocene climate) }\end{array}$ \\
\hline ERL & $\begin{array}{l}\text { of Michigan } \\
\text { Environmental Research }\end{array}$ & ESMAP & $\begin{array}{l}\text { Energy Sector Management } \\
\text { Assistance Programme }\end{array}$ \\
\hline & $\begin{array}{l}\text { Laboratory (Boulder, Colo.) } \\
\text { (NOAA) }\end{array}$ & ESMR & $\begin{array}{l}\text { electronically scanning microwave } \\
\text { radiometer }\end{array}$ \\
\hline ERNets & Energy Research Networks & ESOC & European Space Operations Center \\
\hline $\begin{array}{l}\text { ERNI } \\
\text { EROC }\end{array}$ & $\begin{array}{l}\text { wet-only rainfall collector } \\
\text { Ecological Rates of Change (NSF) }\end{array}$ & ESRC & $\begin{array}{l}\text { Environmental Science Research } \\
\text { Center }\end{array}$ \\
\hline EROS & $\begin{array}{l}\text { Earth Resources Observation } \\
\text { Satellite }\end{array}$ & ESRI & $\begin{array}{l}\text { Environmental Systems Research } \\
\text { Institute }\end{array}$ \\
\hline EROS & $\begin{array}{l}\text { Earth Resources Observation } \\
\text { System }\end{array}$ & ESSA & $\begin{array}{l}\text { U.S. Environmental Science } \\
\text { Services Agency (forenunner of }\end{array}$ \\
\hline EROS & $\begin{array}{l}\text { European River Ocean System } \\
\text { (EEC) }\end{array}$ & ESSC & Earth System Sciences Committee \\
\hline $\begin{array}{l}\text { ERP } \\
\text { ERS }\end{array}$ & $\begin{array}{l}\text { Environmental Research Parks } \\
\text { Economic Research Service }\end{array}$ & ESTAR & $\begin{array}{l}\text { electronically scanned thinned } \\
\text { array radiometer (ARM) }\end{array}$ \\
\hline ERS & ESA Remote Sensing Satellite & ESTEC & $\begin{array}{l}\text { European Space Research and } \\
\text { Technology Centre }\end{array}$ \\
\hline $\begin{array}{l}\text { ERS } \\
\text { ERS-1 }\end{array}$ & $\begin{array}{l}\text { European Remote-Sensing Satellite } \\
\text { European Remote-Sensing } \\
\text { Satellite-1 }\end{array}$ & ESTEC & $\begin{array}{l}\text { European Space Technology } \\
\text { Centre (Netherlands) }\end{array}$ \\
\hline ERS-2 & $\begin{array}{l}\text { European Remote-Sensing } \\
\text { Satellite-2 }\end{array}$ & $\begin{array}{l}\text { ET } \\
\text { ETA } \\
\text { ETAC }\end{array}$ & $\begin{array}{l}\text { evapotranpiration } \\
\text { estimated time of arrival } \\
\text { Environmental Technical }\end{array}$ \\
\hline ERTS & $\begin{array}{l}\text { Earth Resources Technology } \\
\text { Satellite (now Landsat) } \\
\text { Earth Resources Technology }\end{array}$ & & $\begin{array}{l}\text { Applications Center (U.S. Air } \\
\text { Force) }\end{array}$ \\
\hline & $\begin{array}{l}\text { Satellite-1 } \\
\text { Ecological Society of America }\end{array}$ & ETAP & $\begin{array}{l}\text { Expanded Technical Assistance } \\
\text { Program }\end{array}$ \\
\hline $\begin{array}{l}\text { ESA } \\
\text { ESA }\end{array}$ & $\begin{array}{l}\text { Ecological Society of America } \\
\text { Endangered Species Act (1973) }\end{array}$ & ETC & Energy Technology Center \\
\hline ESA & Entomological Society of America & ETC & $\begin{array}{l}\text { European Telecommunications } \\
\text { Standards Institute (EC) }\end{array}$ \\
\hline ESA & $\begin{array}{l}\text { Equatorial South America } \\
\text { (START) }\end{array}$ & etc. & $\begin{array}{l}\text { and others especially of the same } \\
\text { kind; and so forth }\end{array}$ \\
\hline $\begin{array}{l}\text { ESA } \\
\text { ESA/IRS }\end{array}$ & $\begin{array}{l}\text { European Space Agency } \\
\text { European Space }\end{array}$ & $\begin{array}{l}\text { ETF } \\
\text { ETF }\end{array}$ & $\begin{array}{l}\text { Engineered Test Facility } \\
\text { Environmental Task Force }\end{array}$ \\
\hline & $\begin{array}{l}\text { Agency/Information Retrieval } \\
\text { Service }\end{array}$ & ETI & $\begin{array}{l}\text { Economic Transition and } \\
\text { Integration (project) }\end{array}$ \\
\hline ESAD & $\begin{array}{l}\text { Earth Science and Applications } \\
\text { Division (NASA HQ) }\end{array}$ & ETM & $\begin{array}{l}\text { enhanced thematic mapper } \\
\text { (Landsat) }\end{array}$ \\
\hline ESAER & $\begin{array}{l}\text { European Space Agency's Earth } \\
\text { Resources (Satellite) }\end{array}$ & ETRP & $\begin{array}{l}\text { Education and Training } \\
\text { Programme (WMO) }\end{array}$ \\
\hline
\end{tabular}




\section{ILI IL IFAYE ACLONYMS AND ABBRIEVIATIIONS}

ETSA

ETV

EUMELI

EUMETSAT

EUR

EURECA

EUROSTART

EUROTRAC

EUV

EVA

EXCOMM

EZE electron transport system activity

electrothermal vaporizer

eutrophic, mesotrophic, and

oligotrophic sites (France)

(JGOFS)

European Organization for the Exploitation of Meteorological Satellites

Bureau of European Affairs

European Retrievable Carrier

European Planning Committee for START

European Experiment on Transport and Transformation of

Environmentally Relevant Trace Constituents (in the Troposphere over Europe)

extreme ultraviolet

extra vehicular activity

Executive Committee

Asociacion Protestante de

Cooperacion para el Desarrollo en Alemania 


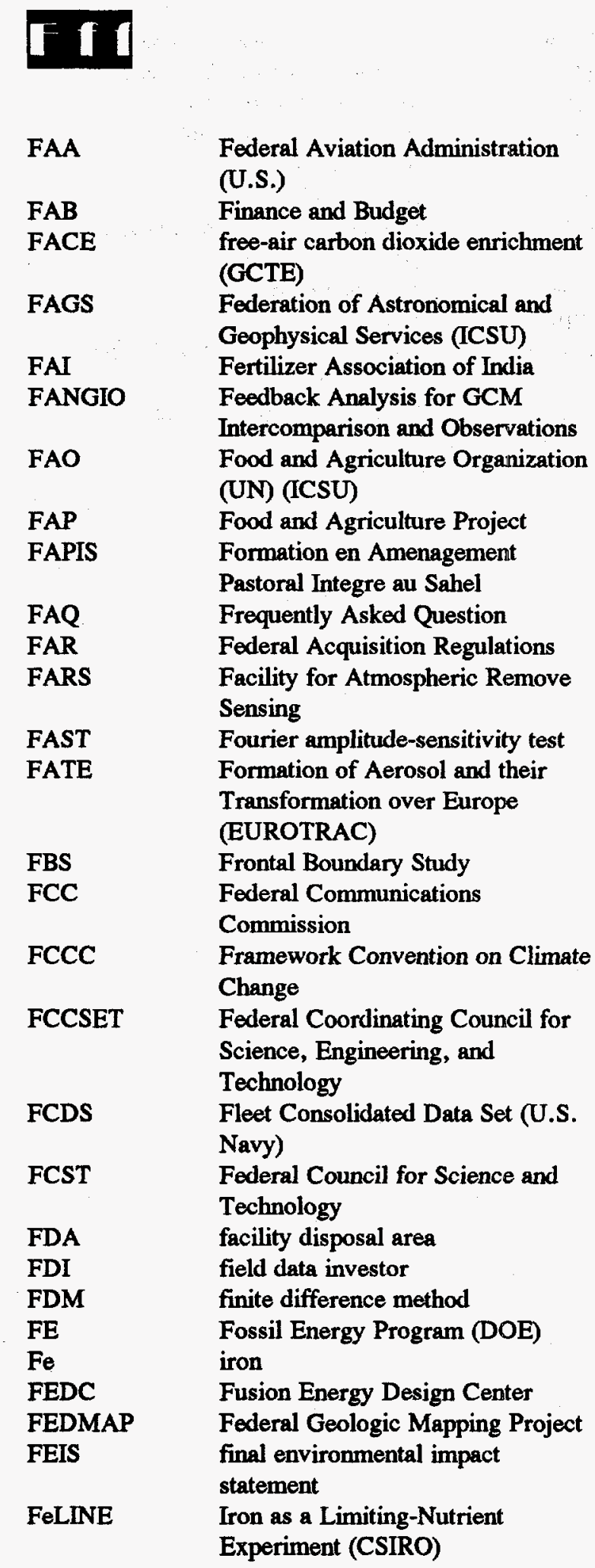

\section{$\mid$}

\begin{tabular}{|c|c|}
\hline FEM & finite element method \\
\hline \multirow[t]{2}{*}{ FEMA } & Federal Emergency Management \\
\hline & Agency (U.S.) \\
\hline \multirow[t]{2}{*}{ FEMA } & Finite Element Model of Material \\
\hline & Transport through Aquifers \\
\hline \multirow[t]{2}{*}{ FERC } & Federal Energy Regulatory \\
\hline & Commission \\
\hline FEWA & $\begin{array}{l}\text { Finite Element of Water Flow } \\
\text { through Aquifers }\end{array}$ \\
\hline FF & free format \\
\hline FFT & fast Fourier transformation \\
\hline \multirow[t]{2}{*}{ FGCC } & Federal Geodetic Control \\
\hline & $\begin{array}{l}\text { Committee (U.S. National Ocean } \\
\text { Service) }\end{array}$ \\
\hline \multirow[t]{2}{*}{ FGDC } & Federal Geographic Data \\
\hline & Commission \\
\hline \multirow{2}{*}{ FGEF } & Federal Geographic Exchange \\
\hline & Format \\
\hline FGGE & $\begin{array}{l}\text { First GARP Global Experiment } \\
\text { (NAS) }\end{array}$ \\
\hline FGGE SOP & FGGE Special Observing Period \\
\hline FICCDC & $\begin{array}{l}\text { Federal Interagency Coordinating } \\
\text { Committee on Digital Cartography } \\
\text { (USGS) }\end{array}$ \\
\hline FICCDC/SWG & $\begin{array}{l}\text { FICCDC/Standards Working } \\
\text { Group (U.S.) }\end{array}$ \\
\hline FID & flame ionization detector (GC) \\
\hline \multirow[t]{2}{*}{ FIELD } & Foundation for International \\
\hline & $\begin{array}{l}\text { Environmental Law and } \\
\text { Development }\end{array}$ \\
\hline FIFE & First ISLSCP Field Experiment \\
\hline FIFRA & $\begin{array}{l}\text { Federal Insecticide, Fungicide, and } \\
\text { Rodenticide Act }\end{array}$ \\
\hline FIN & $\begin{array}{l}\text { Fish Information Network (data } \\
\text { base) }\end{array}$ \\
\hline FIPS & $\begin{array}{l}\text { Federal Information Processing } \\
\text { Standard }\end{array}$ \\
\hline FIRE & First ISCCP Regional Experiment \\
\hline FIT & Field Investigation Team (EPA) \\
\hline FIZ & Fachinformationszentrum \\
\hline FLAIR & $\begin{array}{l}\text { Measurement of Micronutrient } \\
\text { Absorption and Status }\end{array}$ \\
\hline FLINN & Fiducial Laboratories for an \\
\hline & International Natural Sciences \\
\hline & Network \\
\hline
\end{tabular}


TLC IAL I FAVE ACRONYMS AND AMBDEVIATIONS

FLM

FLUPAC

FMFR

FMFT

FNOC

FNWC

FO

FOAM

FOLD

FOR

FORAST

FORET

FORTE

FORTRAN

FOT

FOT

FOV

FPD

$F R$

FRG

FRI

FRLAB

FRONTAL

FRR

FRRPA

FS

FSI

FSL

FSSP

FST

FSU

FTIR

FTIR

FTP federal land manager (e.g., National Park Service)

flux dans l'ouest du Pacifique equatorial (France) (JGOFS)

four-minus-five restoral

four-minus-five test

Fleet Numerical Oceanographic

Center (U.S. Navy)

Fleet Numerical Weather Center (U.S. Navy)

furnace oil

Fates of Aromatic Model

federally owned Landsat data

Forestry Resources (project)

Forest Response to Anthropogenic Stress (model)

Forest of East Tennessee (model) fast on-orbit recording of transient events

FORmula TRANslation

(a computer language)

flight operations team

Fourier Optical Transform

field-of-view (of vision)

flame photometric detector

Federal Register (use with

numbers in text; use "Fed. Regis."

in reference lists)

Federal Republic of Germany

Forest Research Institute

Front Range Lidar, Aircraft, and

Balloon (experiment)

frontal process studies (France)

(JGOFS)

fast repetition rate

Forest and Rangeland Renewable

Planning Act (1974)

Forest Service (USDA)

Forest Survey of India

Forecast Systems Laboratory (ERL and NOAA)

forward scattering spectrometer probe

field support terminal

Florida State University (Miami)

Fourier transform infrared

Fourier transform infrared

radiometer (spectroscopy)

file transfer protocol
FTP/A

FTS

FTS

FTU

FUA

FW

FWCA

FWM

FWP

FWPCA

FWS

FY field task proposa1/agreement Flourier transform spectrometer full turbulence simulation

Formazin turbidity unit

Fuel Use Act

filter wheel

Fish and Wildlife Coordination Act (1958)

food web model

Field Work Proposal

Federal Water Pollution Control

Act (1972)

Fish and Wildlife Service (U.S.)

fiscal year 


G-1
g
GA
GAC
GAGE

GAIM

GALE

GAME

GAMETAG

GARP

GATE

GATE

GATT

GAW

GBF/DIME

Gbps

GBSRN

$G C$

GCC

GCCD

GCDB

GCDIS

GCEP

GCHC

GCIP

GCM

GCM
Gulfstream-1 (airplane)

gram

geographic analysis

global area coverage

Global Atmospheric Gases

Experiment

Global Analysis, Interpretation, and Modelling (IGBP)

Genesis of Atlantic Lows

Experiment

GEWEX-related Asian Monsoon

Experiment

Global Atmospheric Measurements

Experiment of Tropospheric

Aerosols and Gases

Global Atmospheric Research

Program (WMO, ICSU, and

NOAA)

GARP Atlantic Tropical

Experiment

Global Atmosphere Tropical

Experiment

General Agreement on Tariffs and

Trade

Global Atmosphere Watch (WMO)

Geographic Base Files/Dual

Independent Map Encoding (U.S.

Census Bureau)

gigabits per second

Global Baseline Surface Radiation

Network

gas chromatography

global change category

Global Climate Change Digest

(CEI)

Geographic Coordinates Data Base

Global Change Data and

Information System

Global Change Education Program

Gulf Coast Hydroscience Center

(USGS)

GEWEX Continental-Scale

International Project

General Circulation Model

Global Climate Model
GC/MS gas chromatography/mass

spectrometry

GCO

GCOS

GCP

GCPI

GCRIO

GCRP

GCSS

GCSS

GCTE

GCTE-SSC

GCTM

GCRIO

GCSS

GCTE

GCV

GDAAC

GDAS

GDP

GDPS

GDR

GEBA

GEC

GEDEX

GEF

Generation Certification Official

(waste generation)

Global Climate Observing System (WMO, IOC, UNEP, and ICSU)

Ground Control Point

Cape Grim Photochemical

Intensive (CSIRO)

Global Change Research

Information Office

Global Change Research Program

GEWEX Cloud System Study

Global Cloud Systems Study

Global Change and Terrestrial

Ecosystems (program) (IGBP)

GCTE Scientific Steering

Committee

Global Chemical Transport Model

Global Change Research

Information Office

Global Cloud Systems Study

Global Change in Terrestrial

Ecosystems

gross calorific value

Goddard Distributed Active

Archive Center

Grimco data acquisition system

gross domestic product

Global Data-Processing System

(WMO and WWW)

Geophysical Data Record

Global Energy Balance Archive

global environment change

Greenhouse Effect Detection

Experiment

Global Environmental Facility

(WB, UNDP, and UNEP)

GEIA

Global Emissions Inventory

Activity (IGAC)

GEISA Gestion et d'Etude des Information Spectroscopiques Atmospheriques

GEM

GEM-HEX
Geospace Environment Modeling

Geochemical, Microbiological, and Hydrological Experiment 
GLI-IL C F-IVE ACRONYMS AND ABBRREVIATIDNS

\begin{tabular}{|c|c|c|c|}
\hline GEMS & $\begin{array}{l}\text { Global Environmental Monitoring } \\
\text { System (UNEP) }\end{array}$ & GISP II & $\begin{array}{l}\text { Second Greenland Ice Sheet } \\
\text { Project (U.S.) }\end{array}$ \\
\hline GENESIS & $\begin{array}{l}\text { Global Environmental and } \\
\text { Ecological Simulation of }\end{array}$ & GISS & $\begin{array}{l}\text { Goddlard Institute for Space Studies } \\
\text { (NASA) }\end{array}$ \\
\hline & Interactive Systems & GIT & gastrointestinal tract \\
\hline GENLEAF & generic models of leaf response & GIT & Georgia Institute of Technology \\
\hline GEO & Geostationary Earth Observation & & (Atlanta) \\
\hline GEOM & geographically detailed model & GJ & giga joule \\
\hline GEOS & $\begin{array}{l}\text { Geodynamics Experimental Ocean } \\
\text { Satellite }\end{array}$ & $\begin{array}{l}\text { GL } \\
\text { GLAS }\end{array}$ & $\begin{array}{l}\text { Geophysics Laboratory } \\
\text { Geoscience Laser Altimeter }\end{array}$ \\
\hline Geos-3 & Geodetic Satellite Mission (NASA) & & System (formerly GLRS-A) \\
\hline Geosat & $\begin{array}{l}\text { Geologic Satellite (program) } \\
\text { (USN) }\end{array}$ & GLAS & $\begin{array}{l}\text { Goddard Laboratory of } \\
\text { Atmospheric Sciences }\end{array}$ \\
\hline GEOSECS & Geochemical Ocean Sections Study & GLBC & Great Lakes Basin Commission \\
\hline GER & $\begin{array}{l}\text { Global Environmental Research } \\
\text { (U.K.) }\end{array}$ & $\begin{array}{l}\text { GLI } \\
\text { GLIS }\end{array}$ & $\begin{array}{l}\text { global imager } \\
\text { Global Land Information System }\end{array}$ \\
\hline GESAMP & $\begin{array}{l}\text { Group of Experts on the Scientific } \\
\text { Aspects of Marine Pollution }\end{array}$ & $\begin{array}{l}\text { GLL } \\
\text { GLOBE }\end{array}$ & $\begin{array}{l}\text { Galileo } \\
\text { Global Land One kilometer Base }\end{array}$ \\
\hline GEWEX & $\begin{array}{l}\text { Global Energy and Water Cycle } \\
\text { Experiment (WCRP) }\end{array}$ & GLOBE & $\begin{array}{l}\text { Elevation (DIS) } \\
\text { Global Learning and Observations }\end{array}$ \\
\hline GFDL & Geophysical Fluid Dynamics & & to Benefit the Environment \\
\hline GFDL & $\begin{array}{l}\text { Laboratory (NOAA) } \\
\text { Goddard Fluid Dynamics }\end{array}$ & GLOBEC & $\begin{array}{l}\text { Global Ocean Ecosystems } \\
\text { Dynamics (SCOR) }\end{array}$ \\
\hline GFLOPS & $\begin{array}{l}\text { Laboratory } \\
\text { one gigaflops equals } 1 \text { billion }\left(10^{9}\right)\end{array}$ & GLOBEC & $\begin{array}{l}\text { Global Marine Ecosystem } \\
\text { Dynamics (program) }\end{array}$ \\
\hline & $\begin{array}{l}\text { floating point operations per } \\
\text { second (measure of computer }\end{array}$ & GLOCARB & \\
\hline GFO & Grocessing capabi & SHAN & Global Change Research in the \\
\hline $\begin{array}{l}\text { GGI } \\
\text { GGI }\end{array}$ & $\begin{array}{l}\text { GPS geoscience instrument } \\
\text { greenhouse-gas indices }\end{array}$ & GLOCHEM & Global Atmospheric Chemical \\
\hline GGS & global geospace science & GloED & $\begin{array}{l}\text { Survey (IGAC) } \\
\text { Global Emissions Database }\end{array}$ \\
\hline GHCN & $\begin{array}{l}\text { Global Historical Climatology } \\
\text { Network }\end{array}$ & GLONET & $\begin{array}{l}\text { Global Tropospheric Ozone } \\
\text { Network (IGAC) }\end{array}$ \\
\hline $\begin{array}{l}\text { GHG } \\
\text { GHz }\end{array}$ & $\begin{array}{l}\text { greenhouse gas } \\
\text { gigahertz }\end{array}$ & GLRC & Great Lakes Research Center (LSC \\
\hline GIF & Graphical Interchange Format & & and $\mathrm{a}$ \\
\hline GIM & $\begin{array}{l}\text { Global Integration and Modeling } \\
\text { (IGAC) }\end{array}$ & GLRD & Geoscience Laser Ranging System \\
\hline GIMMS & $\begin{array}{l}\text { Global Inventory Modeling and } \\
\text { Monitoring Study }\end{array}$ & GLRS-A & $\begin{array}{l}\text { Geoscience Laser Ranging System- } \\
\text { Altimeter (now GLAS) }\end{array}$ \\
\hline GIS & $\begin{array}{l}\text { geographic information system } \\
\text { (a system for management, } \\
\text { manipulation, analysis, and display } \\
\text { of spatial data) }\end{array}$ & GMCC & $\begin{array}{l}\text { Geoscience Laser Ranging System- } \\
\text { Ranger } \\
\text { Geophysical Monitoring for } \\
\text { Climate Change }\end{array}$ \\
\hline GISMO & $\begin{array}{l}\text { graphic-information-system } \\
\text { modeling }\end{array}$ & GMDSS & $\begin{array}{l}\text { Global Maritime Distress and } \\
\text { Safety System (IMO) }\end{array}$ \\
\hline GISP & $\begin{array}{l}\text { Greenland Ice Sheet Project (NSF } \\
\text { and PAGES) }\end{array}$ & $\begin{array}{l}\text { GMP } \\
\text { GMS }\end{array}$ & $\begin{array}{l}\text { genetically modified plants } \\
\text { Geostationary Meteorological } \\
\text { Satellite (Japan) }\end{array}$ \\
\hline ISP2 2 & Greenland Ice Sheet Pr & & \\
\hline
\end{tabular}




\begin{tabular}{|c|c|c|c|}
\hline $\begin{array}{l}\text { GMT } \\
\text { GNIS }\end{array}$ & $\begin{array}{l}\text { Geologic Map Standards } \\
\text { Committee (USGS) } \\
\text { Greenwich Mean Time } \\
\text { Geographic Names Information } \\
\text { System (USGS) }\end{array}$ & $\begin{array}{l}\text { GR } \\
\text { GRASS }\end{array}$ & $\begin{array}{l}\text { growth rate } \\
\text { Geographic Resources Analysis } \\
\text { Support System (U.S. Army Corps } \\
\text { of Engineers) } \\
\text { Global Runoff Data Centre }\end{array}$ \\
\hline GOALS & $\begin{array}{l}\text { Global Ocean-Atmosphere-Land } \\
\text { System (program) }\end{array}$ & GRECA & $\begin{array}{l}\text { Group of Experts on Accident } \\
\text { Consequences (NEA and OECD) }\end{array}$ \\
\hline GOED & $\begin{array}{l}\text { Global Oceans Ecosystems } \\
\text { Dynamics }\end{array}$ & GREENTIE & $\begin{array}{l}\text { Greenhouse Gas Technology } \\
\text { Information Exchange }\end{array}$ \\
\hline GOES & $\begin{array}{l}\text { Geostationary Operational } \\
\text { Environmental Satellite }\end{array}$ & GRGS & $\begin{array}{l}\text { Groupe de Recherches de Geodesic } \\
\text { Spatiale (France) }\end{array}$ \\
\hline GOES & $\begin{array}{l}\text { Geosynchronous Operational } \\
\text { Environmental Satellite }\end{array}$ & GRID & $\begin{array}{l}\text { Global Resource Information } \\
\text { Database (UNEP) }\end{array}$ \\
\hline GOES & $\begin{array}{l}\text { Global Oceans Ecosystems } \\
\text { Dynamics }\end{array}$ & GRIP & $\begin{array}{l}\text { Greenland Ice Project (PAGES) } \\
\text { (Europe) }\end{array}$ \\
\hline GOES & $\begin{array}{l}\text { Global Omnibus Environmental } \\
\text { Survey (HDGEC) }\end{array}$ & $\begin{array}{l}\text { GRIMCO } \\
\text { GRO }\end{array}$ & $\begin{array}{l}\text { CGBAPS computing system } \\
\text { Gamma Ray Observatory (NASA) }\end{array}$ \\
\hline GOEZS & $\begin{array}{l}\text { Global Ocean Euphotic Zone Study } \\
\text { (IGBP, SCOR, and WCRP) }\end{array}$ & $\begin{array}{l}\text { GSA } \\
\text { GSA }\end{array}$ & $\begin{array}{l}\text { General Services Administration } \\
\text { Geological Society of America }\end{array}$ \\
\hline GOF & Global Ocean Flux & GS\&M & Modeling (task team) \\
\hline $\begin{array}{l}\text { GOFS } \\
\text { GOI }\end{array}$ & $\begin{array}{l}\text { Global Ocean Flux Study } \\
\text { government of India }\end{array}$ & GSC & $\begin{array}{l}\text { Geological Survey of Canada } \\
\text { (DEMR) }\end{array}$ \\
\hline GOIN & $\begin{array}{l}\text { Global Observation Information } \\
\text { Network } \\
\text { Global Ozone Monitoring }\end{array}$ & GSF & $\begin{array}{l}\text { Gesellschaft fur Strahlen und } \\
\text { Umweltforschung mbH Munchen } \\
\text { (Germany) }\end{array}$ \\
\hline GOMI & $\begin{array}{l}\text { Experiment } \\
\text { Global Ozone Monitoring }\end{array}$ & GSFC & $\begin{array}{l}\text { Goddard Space Flight Center } \\
\text { (NASA) }\end{array}$ \\
\hline GOMOS & $\begin{array}{l}\text { Instrument } \\
\text { Global Ozone Monitoring by } \\
\text { Occultation of Stars }\end{array}$ & $\begin{array}{l}\text { GSL } \\
\text { GSMNP }\end{array}$ & $\begin{array}{l}\text { Gulf Stream Locale } \\
\text { Great Smoky Mountains National } \\
\text { Park }\end{array}$ \\
\hline GOMR & $\begin{array}{l}\text { global ozone monitoring } \\
\text { radiomater }\end{array}$ & $\begin{array}{l}\text { GSN } \\
\text { GSP }\end{array}$ & $\begin{array}{l}\text { Global Seismic Network } \\
\text { Greenland Sea Project }\end{array}$ \\
\hline GONG & Global Oscillation Network Group & GSPDC & Geostationary Satellite \\
\hline GoOS & $\begin{array}{l}\text { Global Ocean Observing System } \\
\text { (IOC, UNESCO, WMO, and } \\
\text { ICSU) }\end{array}$ & GSRN & $\begin{array}{l}\text { Precipitation Data Centre } \\
\text { Global Surface Radiation Network } \\
\text { gigation (1,000 million tons) }\end{array}$ \\
\hline GOOS & $\begin{array}{l}\text { Global Ozone Observing System, } \\
\text { (IOC, UNESCO, WMO, and } \\
\text { ICSU) }\end{array}$ & $\begin{array}{l}\text { GtC } \\
\text { GTCE }\end{array}$ & $\begin{array}{l}\text { gigatons (or gigatonnes) of carbon } \\
\text { Global Tropospheric Chemistry } \\
\text { Experiment }\end{array}$ \\
\hline GOS & $\begin{array}{l}\text { Global Observing System (WMO } \\
\text { and WWW) }\end{array}$ & $\begin{array}{l}\text { GTE } \\
\text { GTOS }\end{array}$ & $\begin{array}{l}\text { Global Tropospheric Experiment } \\
\text { Global Terrestrial Observing } \\
\text { System }\end{array}$ \\
\hline $\begin{array}{l}\text { GPC } \\
\text { GPCC }\end{array}$ & Global Processing Center & GTS & Global Telecommunications \\
\hline GPCP & $\begin{array}{l}\text { Center (data base) } \\
\text { Global Precipitation Climatology } \\
\text { Project }\end{array}$ & $\begin{array}{l}\text { GUI } \\
\text { GVap }\end{array}$ & $\begin{array}{l}\text { System (WMO and WWW) } \\
\text { graphical user interface } \\
\text { GEWEX Water Vapor Project } \\
\text { ("GVaP" is preferred)) }\end{array}$ \\
\hline $\begin{array}{l}\text { GPI } \\
\text { GPO }\end{array}$ & $\begin{array}{l}\text { GOES Precipitation Index } \\
\text { Government Printing Office (U.S.) }\end{array}$ & GVaP & $\begin{array}{l}\text { GEWEX Water Vapor Project } \\
\text { (preferred over "GVap") }\end{array}$ \\
\hline $\begin{array}{l}\text { GPS } \\
\text { GPSDR }\end{array}$ & $\begin{array}{l}\text { Global Positioning System } \\
\text { Global Positioning System } \\
\text { Demonstration Receiver }\end{array}$ & $\begin{array}{l}\text { GWE } \\
\text { GWh } \\
\text { GWP }\end{array}$ & $\begin{array}{l}\text { Global Weather Experiment } \\
\text { giga watt hour } \\
\text { greenhouse warming potential }\end{array}$ \\
\hline
\end{tabular}




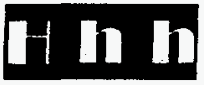

\begin{tabular}{|c|c|}
\hline $\mathbf{H}_{2}$ & hydrogen \\
\hline $\mathrm{H}_{2} \mathrm{O}$ & water \\
\hline $\mathrm{H}_{2} \mathrm{O}_{2}$ & hydrogen peroxide \\
\hline ha & hectare \\
\hline HAAP & high-altitude aerial photograph \\
\hline HAE & height above ellipsoid \\
\hline HAGE & $\begin{array}{l}\text { human activity and global } \\
\text { environment }\end{array}$ \\
\hline HALOE & Halogen Occultation Experiment \\
\hline HALSS & high-altitude lidar sensing station \\
\hline HAO & $\begin{array}{l}\text { High Altitude Observatory } \\
\text { (NCAR) }\end{array}$ \\
\hline HAP & hazardous air pollutant \\
\hline HAPEX & $\begin{array}{l}\text { Hydrological-Atmospheric Pilot } \\
\text { Experiment (GEWEX) }\end{array}$ \\
\hline $\begin{array}{l}\text { HAPEX- } \\
\text { MOBILHY }\end{array}$ & $\begin{array}{l}\text { HAPEX-MOdelisation du BILan } \\
\text { HYdrique }\end{array}$ \\
\hline HARM & $\begin{array}{l}\text { Hazard Assessment Rating } \\
\text { Methodology }\end{array}$ \\
\hline HASL & Health and Safety Laboratory \\
\hline HASP & High Altitude Sampling Program \\
\hline HBJ & Hazira-Bijapur-Jagdishpur (India) \\
\hline HBL & Hudson Bay Lowland \\
\hline $\mathrm{HBr}$ & hydrogen bromide \\
\hline HBW & half band-width \\
\hline HCBP & hexachlorobiphenol \\
\hline HCFC & hydrochlorofluorocarbon \\
\hline $\mathrm{HCHO}, \mathrm{CH}_{2} \mathrm{O}$ & formaldehyde \\
\hline $\mathrm{HCl}$ & hydrogen chloride \\
\hline HCMM & Heat-Capacity Mapping Mission \\
\hline HCMM/AEM-1 & $\begin{array}{l}\text { HCMM/Applications Explorer } \\
\text { Mission-1 }\end{array}$ \\
\hline HCMR & heat-capacity mapping radiometer \\
\hline HCN & Historical Climatology Network \\
\hline HCN & hydrogen cyanide \\
\hline HCND & $\begin{array}{l}\text { Historical Climatology Network- } \\
\text { Daily }\end{array}$ \\
\hline HD & hierarchical diagnosis \\
\hline HDF & hierarchical data format \\
\hline HDGCB & $\begin{array}{l}\text { Human Dimensions of Global } \\
\text { Change (programme) (UNU, } \\
\text { ISSC, and IFIAS) }\end{array}$ \\
\hline HDGI & $\begin{array}{l}\text { Human Dimensions of Global } \\
\text { Environmental Change } \\
\text { (programme) (ISSC) }\end{array}$ \\
\hline
\end{tabular}

\begin{tabular}{|c|c|}
\hline HDP & $\begin{array}{l}\text { Human Dimensions Program } \\
\text { (SEDAC) }\end{array}$ \\
\hline HDP & $\begin{array}{l}\text { Human Dimensions of Global } \\
\text { Environmental Change Program } \\
\text { (ISSC) }\end{array}$ \\
\hline HDRL & High-Dose Reference Laboratory \\
\hline HDTV & High-Definition Television \\
\hline HEC & Hydro-Electric Commission \\
\hline HECP & $\begin{array}{l}\text { Herbaceous Energy Crops } \\
\text { Program }\end{array}$ \\
\hline HEIFE & Heihe River Field Experiment \\
\hline HELCOM & Helsinki Commission \\
\hline HEM & $\begin{array}{l}\text { Harmonizations of Environmental } \\
\text { Measurement (program) }\end{array}$ \\
\hline HESS & $\begin{array}{l}\text { High Latitude Ecosystems as } \\
\text { Sources and Sinks of Trace Gases } \\
\text { (IGAC) }\end{array}$ \\
\hline HEW & Health, Education, and Welfare \\
\hline HF & hydrogen fluoride \\
\hline HFC & hydrofluorocarbon \\
\hline HHMS & $\begin{array}{l}\text { Hydrostatic Head Monitoring } \\
\text { Station (program) }\end{array}$ \\
\hline HHS & $\begin{array}{l}\text { Department of Health and Human } \\
\text { Services }\end{array}$ \\
\hline HI & human interactions \\
\hline HIC & Habitat International Coalition \\
\hline HLDA & $\begin{array}{l}\text { high-latitude diffusive-advective } \\
\text { model }\end{array}$ \\
\hline HMMS & $\begin{array}{l}\text { high-resolution microwave } \\
\text { spectrometer sounder (now MIMR) }\end{array}$ \\
\hline HIRDLS & $\begin{array}{l}\text { high-resolution dynamics limb } \\
\text { sounder }\end{array}$ \\
\hline HIRIS & $\begin{array}{l}\text { high-resolution imaging } \\
\text { spectrometer }\end{array}$ \\
\hline HIRS & $\begin{array}{l}\text { high-resolution infrared radiation } \\
\text { sounder }\end{array}$ \\
\hline HIS & $\begin{array}{l}\text { high-resolution interferometer } \\
\text { sounder }\end{array}$ \\
\hline HIS & $\begin{array}{l}\text { high-spectral resolution } \\
\text { interferometer sounder }\end{array}$ \\
\hline HL̇Q & $\begin{array}{l}\text { holocellulose/lignocellulose } \\
\text { quotient }\end{array}$ \\
\hline HLW & high-level waste \\
\hline HMCRI & $\begin{array}{l}\text { Hazardous Materials Control } \\
\text { Research Institute }\end{array}$ \\
\hline
\end{tabular}




\begin{tabular}{|c|c|c|c|}
\hline HMMR & $\begin{array}{l}\text { high-resolution multifrequency } \\
\text { microwave radiometer } \\
\text { high-resolution microwave } \\
\text { sounding unit }\end{array}$ & $\begin{array}{l}\text { HSWA } \\
\text { html } \\
\text { http }\end{array}$ & $\begin{array}{l}\text { Hazardous and Solid Waste } \\
\text { Amendments (1984) } \\
\text { hypertext markup language } \\
\text { hypertext transport protocol }\end{array}$ \\
\hline HMTA & $\begin{array}{l}\text { Hazardous Materials } \\
\text { Transportation Act }\end{array}$ & HUD & $\begin{array}{l}\text { Department of Housing and Urban } \\
\text { Development (U.S.) }\end{array}$ \\
\hline HMW & high molecular weight & HVI & high-volume impactor \\
\hline $\mathrm{HNO}_{3}$ & nitric acid & HVS & high-volume sampler \\
\hline HNRC & HOMS National Reference Centre & HWRP & Hydrology and Water Resources \\
\hline $\begin{array}{l}\mathrm{HO}_{\mathbf{x}} \\
\mathrm{HOC} \\
\mathrm{HOCl}\end{array}$ & $\begin{array}{l}\text { odd hydrogen }\left(\mathrm{OH}, \mathrm{HO}_{2} \text {, and }\right. \\
\left.\mathrm{H}_{2} \mathrm{O}_{2}\right) \\
\text { hydrophobic organic compounds } \\
\text { hypochlorous acid }\end{array}$ & HYNET & $\begin{array}{l}\text { Programme (WMO) } \\
\text { Intercomparison of Operational } \\
\text { Hydrological Network Design } \\
\text { Techniques }\end{array}$ \\
\hline $\begin{array}{l}\text { HUCl } \\
\text { HOMS }\end{array}$ & $\begin{array}{l}\text { Hydrological Operational } \\
\text { Multipurpose Subprogramme } \\
\text { (OHP and WMO) }\end{array}$ & Hz & $\begin{array}{l}\text { Techniques } \\
\text { hertz (cycles per second) }\end{array}$ \\
\hline HOT & Hawaii ocean time (series) & & \\
\hline $\begin{array}{l}\text { HP } \\
\text { HP }\end{array}$ & $\begin{array}{l}\text { health physicist } \\
\text { health physics }\end{array}$ & & \\
\hline HP & Hewlett-Packard & & \\
\hline HPCC & $\begin{array}{l}\text { High Performance Computing and } \\
\text { Communications }\end{array}$ & & \\
\hline HPCRC & $\begin{array}{l}\text { High Performance Computing } \\
\text { Research Center (ORNL) }\end{array}$ & & \\
\hline HPD & $\begin{array}{l}\text { Hydrometeorological Processes } \\
\text { Division }\end{array}$ & & \\
\hline HPLC & $\begin{array}{l}\text { high-precision liquid } \\
\text { chromatography }\end{array}$ & & \\
\hline HPLC & $\begin{array}{l}\text { high-pressure liquid } \\
\text { chromatograph/chromatography }\end{array}$ & & \\
\hline HPS & $\begin{array}{l}\text { Health Physics Society (Bethesda, } \\
\text { Md.) }\end{array}$ & & \\
\hline HQ & headquarters & & \\
\hline HRDI & high-resolution Doppler imager & & \\
\hline HRE & $\begin{array}{l}\text { Homogeneous Reactor Experiment } \\
\text { (impoundment) }\end{array}$ & & \\
\hline HRIRS & $\begin{array}{l}\text { high-resolution infrared radiation } \\
\text { sounder }\end{array}$ & & \\
\hline HROI & high-resolution optical instrument & & \\
\hline HRPT & $\begin{array}{l}\text { high-resolution picture } \\
\text { transmission (AVHRR) }\end{array}$ & & \\
\hline HRS & Hazard Ranking System (EPA) & & \\
\hline HRV & high resolution video (SPOT) & & \\
\hline HRV & high resolution visible & & \\
\hline HSA & Historic Sites Act (1935) & & \\
\hline HSL & Hazardous Substance List (EPA) & & \\
\hline HSRL & High Spectral Resolution Lidar & & \\
\hline HST & Hawaii standard time & & \\
\hline HST & Hubble Space Telescope & & \\
\hline
\end{tabular}




\section{TLI IL IFAVE ACRONYMS AND ABBRIEVIATIDNS}

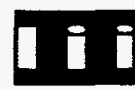

$3 I$

IAA

IAC

IACG

IAEA

IAEG

IAF

IAG

IAGA

IAGC

IAH

IAHR

IAHS

IAI

IAL

IAMAP

IAMAP-IAHS

IAMAS

IAOPA

IAP

IAPSO

IAS

IASC improved initialization inversion

International Academy of

Astronautics

International Activities Committee

Interagency Committee on

Geomatics (DEMR)

International Atomic Energy

Agency (UN)

International Association for

Engineering Geology

International Astronautical

Federation

International Association of

Geodesy (IUGG)

International Association of

Geomagnetism and Agronomy

(IUGG)

International Association of

Geochemistry and Cosmochemistry

International Association of

Hydrogeologists (IUGS)

International Association for

Hydraulic Research

International Association of

Hydrological Sciences (IUGG)

Inter-American Institute for Global

Change Research (START)

International Association of

Theoretical Applied Limnologists

International Association of

Meteorology and Atmospheric

Physics (IUGG)

IAMAP-International Association

of Hydrological Sciences (IUGG)

International Association of

Meteorology and Atmospheric

Sciences (IUGG and ICSU)

International Aircraft Owners and

Pilots Association

Institute of Atmospheric Physics

International Association for

Physical Sciences of the Ocean

(IUGG)

Image Analysis System

International Arctic Sciences

Committee
IASI

IASI

IASPEI

IASTED

IATA

IATUL

IAU

IAVCEI

IAWPRC

IBM

IBM PC

IBP

IBP

IBRD

IBSRAM

IBSS

IBWC

IC

IC

IC

IC

ICA infrared atmospheric sounding instrument

infrared atmospheric sounding interferometer

International Association of Seismology and Physics of the Earth's Interior (IUGG)

International Association of

Science and Technology for

Development

International Air Transport

Association

International Association of

Technological University Libraries

International Astronomical Union

International Association of

Volcanology and Chemistry of the

Earth's Interior (IUGG)

International Association on Water

Pollution Research and Control

International Business Machines

(Corp.)

International Business Machines

Personal Computer

International Biological

Programme (ICSU)

International Biophysical Program

International Bank for

Reconstruction and Development

International Board for Soil

Research and Management

Institute of Biology of the Southern

Seas (Russian Federation; formerly

U.S.S.R.)

International Boundary and Water

Commission (Independent; U.S.

and Mexico)

industrialized country

interaction coefficient

Intergovernmental Council

ion chromatography

International Communication

Agency 
(-LI I- IL I FAT-E ACLONYMS ANID AIBIBIEVIATIIONS

\begin{tabular}{|c|c|c|c|}
\hline \multirow[t]{2}{*}{ ICACGP } & $\begin{array}{l}\text { International Commission on } \\
\text { Atmospheric Chemistry and Global }\end{array}$ & ICOLD & $\begin{array}{l}\text { International Commission on Large } \\
\text { Dams }\end{array}$ \\
\hline & Pollution (IAMAS) & ICP & inductively coupled plasma \\
\hline ICALPE & $\begin{array}{l}\text { International Centre for Alpine } \\
\text { Environments (Chambery, France) }\end{array}$ & & $\begin{array}{l}\text { emission (spectroscopy) } \\
\text { inductively coupled plasma/mass }\end{array}$ \\
\hline ICAO & $\begin{array}{l}\text { International Civil Aviation } \\
\text { Organization }\end{array}$ & ICP-OES & $\begin{array}{l}\text { spectrometry } \\
\text { inductively coupled }\end{array}$ \\
\hline ICAR & $\begin{array}{l}\text { Indian Council of Agricultural } \\
\text { Research }\end{array}$ & & $\begin{array}{l}\text { plasma-optical emission } \\
\text { spectroscopy }\end{array}$ \\
\hline ICAR & $\begin{array}{l}\text { International Center for Action } \\
\text { Research }\end{array}$ & $\begin{array}{l}\text { ICPP } \\
\text { ICRAF }\end{array}$ & $\begin{array}{l}\text { Idaho Chemical Processing Plant } \\
\text { International Council for Research }\end{array}$ \\
\hline ICARDA & $\begin{array}{l}\text { International Center for } \\
\text { Agricultural Research in Dry } \\
\text { Areas }\end{array}$ & ICRCCM & $\begin{array}{l}\text { in Agroforestry } \\
\text { Intercomparison of Radiation } \\
\text { Codes in Climate Models }\end{array}$ \\
\hline ICAS & $\begin{array}{l}\text { International Council of the } \\
\text { Aeronautical Sciences }\end{array}$ & ICRM & $\begin{array}{l}\text { (program) } \\
\text { International Committee on }\end{array}$ \\
\hline ICASVR & $\begin{array}{l}\text { International Committee on } \\
\text { Atmosphere-Soil-Vegetation } \\
\text { Relations (IAHS) }\end{array}$ & ICRP & $\begin{array}{l}\text { Radionuclide Meteorology } \\
\text { International Commission on } \\
\text { Radiological Protection }\end{array}$ \\
\hline ICCE & $\begin{array}{l}\text { Instrument Control Center } \\
\text { International Commission on } \\
\text { Continental Erosion (IAHS) }\end{array}$ & ICRSDT & $\begin{array}{l}\text { International Committee on } \\
\text { Remote Sensing and Data } \\
\text { Transmission (IAHS) }\end{array}$ \\
\hline $\begin{array}{l}\text { ICDB } \\
\text { ICE }\end{array}$ & $\begin{array}{l}\text { Ice Core Data Bank } \\
\text { International Cirrus Experiment }\end{array}$ & ICRU & $\begin{array}{l}\text { International Commission on } \\
\text { Radiological Units }\end{array}$ \\
\hline \multirow{2}{*}{$\begin{array}{l}\text { ICE } \\
\text { ICEAR }\end{array}$} & International Cometary Explorer & ICSC & International Civil Service \\
\hline & $\begin{array}{l}\text { International Centre for Equatorial } \\
\text { Atmospheric Research (Indonesia) }\end{array}$ & & $\begin{array}{l}\text { Commission } \\
\text { International Continental Scientific }\end{array}$ \\
\hline ICES & $\begin{array}{l}\text { International Council for the } \\
\text { Exploration of the Seas }\end{array}$ & & $\begin{array}{l}\text { Drilling (program) } \\
\text { International Commission on Snow }\end{array}$ \\
\hline ICGW & $\begin{array}{l}\text { Instrument Control Facility } \\
\text { International Commission on }\end{array}$ & ICSTI & and Ice (IAHS) \\
\hline ICIC & $\begin{array}{l}\text { Ground Water (IAHS) } \\
\text { Intercalibrations-Intercomparisons } \\
\text { (IGAC) }\end{array}$ & ICSU & $\begin{array}{l}\text { and Technical Information } \\
\text { International Council of Scientific } \\
\text { Unions }\end{array}$ \\
\hline ICICI & $\begin{array}{l}\text { Industrial Credit and Investment } \\
\text { Corporation of India }\end{array}$ & ICSU-ACE & $\begin{array}{l}\text { ICSU Advisory Committee on the } \\
\text { Environment }\end{array}$ \\
\hline ICID & $\begin{array}{l}\text { International Commission on } \\
\text { Irrigation and Drainage }\end{array}$ & ICSW & $\begin{array}{l}\text { International Commission on } \\
\text { Surface Water (IAHS) }\end{array}$ \\
\hline \multirow[t]{2}{*}{ ICIMOD } & $\begin{array}{l}\text { International Centre for Integrated } \\
\text { Mountain Development for the }\end{array}$ & ICTP & $\begin{array}{l}\text { International Centre for Theoretical } \\
\text { Physics }\end{array}$ \\
\hline & $\begin{array}{l}\text { Hindu-Kush Himalayan Region } \\
\text { (Kathmandu, Nepal) }\end{array}$ & ICWG & $\begin{array}{l}\text { International Coordination } \\
\text { Working Group }\end{array}$ \\
\hline ICIP & $\begin{array}{l}\text { International Conference on Image } \\
\text { Processing }\end{array}$ & ICWQ & $\begin{array}{l}\text { International Commission on } \\
\text { Water Quality (IAHS) }\end{array}$ \\
\hline $\begin{array}{l}\text { ICL } \\
\text { ICL }\end{array}$ & $\begin{array}{l}\text { integrated cloud liquid } \\
\text { International-Union Commission }\end{array}$ & ICWRS & $\begin{array}{l}\text { International Commission on } \\
\text { Water Resources Systems (IAHS) }\end{array}$ \\
\hline & on the Lithosphere (ICSU) & IDA & International Development Agency \\
\hline ICLD & $\begin{array}{l}\text { International Commission on Large } \\
\text { Dams }\end{array}$ & IDASS & $\begin{array}{l}\text { Integrated Data Assimilation and } \\
\text { Sounding System }\end{array}$ \\
\hline ICN & ice condensation nuclei & IDB & Inter-American Development Bank \\
\hline
\end{tabular}


T-L I AL G IVIE ACDONYMS AND ABBBREVIATIIONS

\begin{tabular}{|c|c|c|c|}
\hline IDCCC & Interior Department Cartographic & IFREMER & Institut Francais de Recherche \\
\hline & Image Dissector Camers System & & $\begin{array}{l}\text { pour I Exploration de la Mer } \\
\text { Institut Francais pour la Researche }\end{array}$ \\
\hline IDDSAC & Interim Data Documentation & 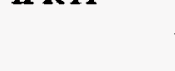 & et la Technologie Polaire (French \\
\hline IDEAL & $\begin{array}{l}\text { Standard for ARC/INFO Averages } \\
\text { International Decade for the East }\end{array}$ & & $\begin{array}{l}\text { Institute for Polar Research } \\
\text { Technology) }\end{array}$ \\
\hline & African Lakes (project) (PAGES) & IFS & Integrated Forest Study on the \\
\hline IDNDR & $\begin{array}{l}\text { International Decade for Natural } \\
\text { Disaster Reduction }\end{array}$ & & $\begin{array}{l}\text { Effects of Atmospheric Deposition } \\
\text { International Foundation for }\end{array}$ \\
\hline IDP & Instrument Development Program & & Science \\
\hline IDRI & $\begin{array}{l}\text { Thailand Development Research } \\
\text { Institute }\end{array}$ & IFYGL & $\begin{array}{l}\text { International Field Year for the } \\
\text { Great Lakes (IHD) }\end{array}$ \\
\hline IDS & interdisciplinary science & IG & Institute of Geography \\
\hline IDSS & Integrated Data Sounding System & IGAC & Internationail Global Atmospheric \\
\hline IDRI & $\begin{array}{l}\text { Thailand Development Research } \\
\text { Institute }\end{array}$ & & $\begin{array}{l}\text { Chemistry (program) (IGBP and } \\
\text { ICACGP) }\end{array}$ \\
\hline IEA & Institute of Energy Analysis & IGAC-SSC & IGAC Scientific Steering \\
\hline IEA & International Energy Agency & & Committee \\
\hline IEAVORAU & $\begin{array}{l}\text { Institute of Energy Analysis/Oak } \\
\text { Ridge Associated Universities }\end{array}$ & IGAP & $\begin{array}{l}\text { International Global Aerosol } \\
\text { Program }\end{array}$ \\
\hline IEC & $\begin{array}{l}\text { Implementation and Effectiveness } \\
\text { of International Environmental } \\
\text { Commitments }\end{array}$ & IGBP & $\begin{array}{l}\text { International Geosphere-Biosphere } \\
\text { Programme (ICSU) }\end{array}$ \\
\hline IEE & Institution of Electrical Engineers & IGBP & IGBP Data and Information System \\
\hline IEEE & $\begin{array}{l}\text { Institute of Electrical and } \\
\text { Electronics Engineers (Inc.) }\end{array}$ & IGC & International Geophysical \\
\hline IEEE & $\begin{array}{l}\text { Institute for Electronics and } \\
\text { Electrical Engineering }\end{array}$ & IGCP & $\begin{array}{l}\text { International Geological } \\
\text { Correlation Programme (IUGS and } \\
\text { UNESCO) }\end{array}$ \\
\hline IELV & $\begin{array}{l}\text { intermediate expendable launch } \\
\text { vehicle }\end{array}$ & IGEA & International Group of Funding \\
\hline IEM & $\begin{array}{l}\text { Institute of Experimental } \\
\text { Meteorology }\end{array}$ & & $\begin{array}{l}\text { Agencies for Global Change } \\
\text { Research }\end{array}$ \\
\hline IEOS & $\begin{array}{l}\text { International Earth Observing } \\
\text { System }\end{array}$ & IGFA & $\begin{array}{l}\text { Inter-Governmental Funding } \\
\text { Agencies }\end{array}$ \\
\hline IERS & $\begin{array}{l}\text { International Earth Reference } \\
\text { System }\end{array}$ & IGFA & $\begin{array}{l}\text { International Group of Funding } \\
\text { Agencies (for Global Change }\end{array}$ \\
\hline IFAD & International Fund for Agricultural & & Research) \\
\hline & Development (UN) & IGLD & International Great Lakes Datum \\
\hline IFDC & International Fertilizer & IGN & Institut Geographique National \\
\hline & Development Center & IGO & Intergovernmental Organization \\
\hline IFE & $\begin{array}{l}\text { Institute for Energy Technology } \\
\text { (Norway) }\end{array}$ & IGOSS & $\begin{array}{l}\text { Integrated Global Ocean Services } \\
\text { System }\end{array}$ \\
\hline IFIAS & $\begin{array}{l}\text { International Federation of } \\
\text { Institutes for Advanced Study }\end{array}$ & IGOSS & $\begin{array}{l}\text { Integrated Global Ocean Station } \\
\text { System (UNESCO) }\end{array}$ \\
\hline IFIM & $\begin{array}{l}\text { Instream Flow Incremental } \\
\text { Methodology (U.S. Fish and }\end{array}$ & IGPO & $\begin{array}{l}\text { International GEWEX Project } \\
\text { Office }\end{array}$ \\
\hline IFO & $\begin{array}{l}\text { Wildlife Service) } \\
\text { intensive field observation }\end{array}$ & IGRSS & $\begin{array}{l}\text { International Geoscience and } \\
\text { Remote Sensing Society }\end{array}$ \\
\hline IFOV & instantaneous field of view & IGS & International Glaciological Society \\
\hline RB & $\begin{array}{l}\text { International Frequency } \\
\text { Registration Board }\end{array}$ & & $\begin{array}{l}\text { Institute of Gas Technology } \\
\text { (Chicago) }\end{array}$ \\
\hline
\end{tabular}




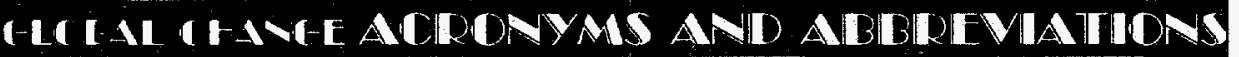

\begin{tabular}{|c|c|c|c|}
\hline IGU & $\begin{array}{l}\text { International Geographical Union } \\
\text { (ICSU) }\end{array}$ & IMO & $\begin{array}{l}\text { International Meteorological } \\
\text { Organization (formerly WMO) }\end{array}$ \\
\hline IGY & $\begin{array}{l}\text { International Geophysical Year } \\
\text { (1 July } 1957 \text { - } 31 \text { December 1958) }\end{array}$ & IMOP & $\begin{array}{l}\text { Instruments and Methods of } \\
\text { Observation Programme (WMO) }\end{array}$ \\
\hline IHB & International Hydrographic Bureau & IMP & ice mass path \\
\hline IHD & $\begin{array}{l}\text { International Hydrological Decade } \\
\text { (UNESCO) }\end{array}$ & $\begin{array}{l}\text { IMS } \\
\text { IMS }\end{array}$ & $\begin{array}{l}\text { Information Management Services } \\
\text { International Mountain Society }\end{array}$ \\
\hline IHO & $\begin{array}{l}\text { International Hydrographic } \\
\text { Organization }\end{array}$ & $\begin{array}{l}\text { IMU } \\
\text { IN }\end{array}$ & $\begin{array}{l}\text { International Mathematical Union } \\
\text { ice nuclei }\end{array}$ \\
\hline IHP & $\begin{array}{l}\text { International Hydrological } \\
\text { Program (UNESCO) }\end{array}$ & $\begin{array}{l}\text { in. } \\
\text { Inc. }\end{array}$ & $\begin{array}{l}\text { inch } \\
\text { Incorporated }\end{array}$ \\
\hline $\begin{array}{l}\text { IHS } \\
\text { IHSS }\end{array}$ & $\begin{array}{l}\text { International Heliospheric Study } \\
\text { International Humic Substances } \\
\text { Society }\end{array}$ & INC & $\begin{array}{l}\text { Intergovernmental Negotiating } \\
\text { Committee (for a Framework }\end{array}$ \\
\hline $\begin{array}{l}\text { II } \\
\text { IIASA }\end{array}$ & $\begin{array}{l}\text { Society } \\
\text { interdisciplinary investigator } \\
\text { Intermational Institute for Applied }\end{array}$ & INC & $\begin{array}{l}\text { Convention on Climate Change) } \\
\text { International Negotiating } \\
\text { Convention }\end{array}$ \\
\hline IIG & $\begin{array}{l}\text { Systems Analysis (Vienna) } \\
\text { Indian Institute of Geomagnetism }\end{array}$ & INCD & $\begin{array}{l}\text { International Convention to } \\
\text { Combat Decertification }\end{array}$ \\
\hline IIID & $\begin{array}{l}\text { International Institute of } \\
\text { Information Design }\end{array}$ & $\begin{array}{l}\text { IND } \\
\text { INEL }\end{array}$ & $\begin{array}{l}\text { Industrial Metabolism Project } \\
\text { Idaho National Engineering }\end{array}$ \\
\hline IIOE & $\begin{array}{l}\text { International Indian Ocean } \\
\text { Expedition }\end{array}$ & INFOCLIMA & $\begin{array}{l}\text { Laboratory } \\
\text { World Climate Data Information }\end{array}$ \\
\hline IISL & $\begin{array}{l}\text { International Institute of Space } \\
\text { Law }\end{array}$ & & $\begin{array}{l}\text { and Referral System } \\
\text { International Referral System for }\end{array}$ \\
\hline IITM & $\begin{array}{l}\text { Indian Institute for Tropical } \\
\text { Meteorology }\end{array}$ & & $\begin{array}{l}\text { Sources of Environmental } \\
\text { Information (UNEP) }\end{array}$ \\
\hline UC & $\begin{array}{l}\text { International Joint Commission } \\
\text { (Independent; Canada and U.S.) }\end{array}$ & INPA & $\begin{array}{l}\text { Instituto Nacional de Pesquisas da } \\
\text { Amazona (Brazil) }\end{array}$ \\
\hline ILAS & $\begin{array}{l}\text { Improved Limb Atmospheric } \\
\text { Spectrometer }\end{array}$ & INPE & $\begin{array}{l}\text { Brazilian Institute for Space } \\
\text { Research (Brazil) }\end{array}$ \\
\hline ILO & International Labor Organization & INPE & Instituto Nacional de Pesquisas \\
\hline 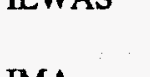 & Acidification Study (EPRI) & INQUA & International Union for Quaternary \\
\hline IM-Al & $\begin{array}{l}\text { Information Management gnd } \\
\text { Archives (Office) } \\
\text { inorganic monomeric aluminum }\end{array}$ & INRA & $\begin{array}{l}\text { Research } \\
\text { Institut National de Recherche } \\
\text { Agronomiques (France) }\end{array}$ \\
\hline IMAGES & $\begin{array}{l}\text { International Marine Global } \\
\text { Change Study (PAGES and SCOR) }\end{array}$ & $\begin{array}{l}\text { INS } \\
\text { INS }\end{array}$ & $\begin{array}{l}\text { inertial navigation system } \\
\text { Institute Scholars }\end{array}$ \\
\hline $\begin{array}{l}\text { IMB } \\
\text { IMC }\end{array}$ & $\begin{array}{l}\text { Investigator of Micro-Biosphere } \\
\text { ice mass content }\end{array}$ & INSA & $\begin{array}{l}\text { Institut National des Sciences } \\
\text { Appliques (France) }\end{array}$ \\
\hline IMCO & $\begin{array}{l}\text { Inter-Governmental Maritime } \\
\text { Consultative Organization }\end{array}$ & $\begin{array}{l}\text { INSAT } \\
\text { INSTARR }\end{array}$ & $\begin{array}{l}\text { Indian Satellite } \\
\text { Institute for Arctic and Alpine }\end{array}$ \\
\hline $\begin{array}{l}\text { IMD } \\
\text { IMF }\end{array}$ & $\begin{array}{l}\text { India Meteorological Department } \\
\text { ice mass flux }\end{array}$ & & $\begin{array}{l}\text { Research (University of Colorado, } \\
\text { Boulder) }\end{array}$ \\
\hline IMF & International Monetary Fund & INSULA & International Scientific Council for \\
\hline IMG & $\begin{array}{l}\text { Interferometric Monitor for } \\
\text { Greenhouse Gases }\end{array}$ & INT & $\begin{array}{l}\text { Island Development (NGO) } \\
\text { p-iodonitrotetrazolium }\end{array}$ \\
\hline IMO & $\begin{array}{l}\text { International Maritime } \\
\text { Organization }\end{array}$ & INTECOL & $\begin{array}{l}\text { International Association of } \\
\text { Ecology (ICSU and IUBS) }\end{array}$ \\
\hline
\end{tabular}


GLIL I IVTE ACDONYMS AND AMBBDEVIAIIDNS

\begin{tabular}{|c|c|c|c|}
\hline INZN & Institut Zairois pour la & IRIS & integrated radar imaging system \\
\hline & Conservation de la Nature & IRP & Installation Restoration Program \\
\hline I/O & input/output & IRRI & International Rice Research \\
\hline IO & input/output & & Institute \\
\hline IOB & $\begin{array}{l}\text { Inter-Organization Board for } \\
\text { Information Systems and Related } \\
\text { Activities }\end{array}$ & $\begin{array}{l}\text { IRSS } \\
\text { IRSSP }\end{array}$ & $\begin{array}{l}\text { Indian Remote Sensing Satellite } \\
\text { Interactive Remote Sensing } \\
\text { Software Package }\end{array}$ \\
\hline IOC & $\begin{array}{l}\text { Intergovernmental Oceanographic } \\
\text { Council (UNESCO) }\end{array}$ & $\begin{array}{l}\text { IRTM } \\
\text { IRTS }\end{array}$ & $\begin{array}{l}\text { infrared thermal mapper } \\
\text { infrared temperature sounder }\end{array}$ \\
\hline IOC & $\begin{array}{l}\text { Intergovernmental Oceanographic } \\
\text { Commission (UNESCO) }\end{array}$ & $\begin{array}{l}\text { IS } \\
\text { ISA }\end{array}$ & $\begin{array}{l}\text { Image Subtraction } \\
\text { Israel Space Agency }\end{array}$ \\
\hline IOC & International Ozone Commission & ISA & Italian Space Agency \\
\hline IOP & intensive observing period & ISAMS & improved stratospheric and \\
\hline IOPG & $\begin{array}{l}\text { Indian Ocean Planning Group } \\
\text { (JGOFS) }\end{array}$ & ISAR & $\begin{array}{l}\text { mesospheric sounder } \\
\text { Intelligent Synthetic Aperture }\end{array}$ \\
\hline IP & independent pixel & & Radar \\
\hline IP & Internet Protocol & ISAS & Institute of Space and Astronautical \\
\hline IPB & $\begin{array}{l}\text { Bogor Agriculture Institute } \\
\text { (Indonesia) }\end{array}$ & ISC & $\begin{array}{l}\text { Science (Japan) } \\
\text { International Seismological Centre }\end{array}$ \\
\hline IPCC & $\begin{array}{l}\text { Intergovernmental Panel on } \\
\text { Climate Change (WMO and }\end{array}$ & ISCCP & $\begin{array}{l}\text { International Satellite Cloud } \\
\text { Climatology Program (WCRP) }\end{array}$ \\
\hline & UNEP) & ISEATRA & International Symposium on \\
\hline IPCC/RSWG & IPCC/Response Strategies & & Ecological Aspects of Tree-Ring \\
\hline /EIS & $\begin{array}{l}\text { Working Group/Energy and } \\
\text { Industry Subgroup }\end{array}$ & ISEB 10 & $\begin{array}{l}\text { Analysis } \\
\text { Tenth International Symposium on }\end{array}$ \\
\hline IPEI & $\begin{array}{l}\text { Ionospheric Plasma and } \\
\text { Electrodynamics Instrument }\end{array}$ & ISEE & $\begin{array}{l}\text { Environmental Biogeochemistry } \\
\text { International Sun-Earth Explorer }\end{array}$ \\
\hline IPMS & International Polar Motion Service & ISEM & International Society for Ecological \\
\hline IPOC & $\begin{array}{l}\text { International Partner Operations } \\
\text { Center }\end{array}$ & ISFA & $\begin{array}{l}\text { Modelling } \\
\text { International Scientific Film }\end{array}$ \\
\hline IPR & Intellectual Property Right & & Association \\
\hline IPS & Inter Press Service & ISI & International Statistical Institute \\
\hline IPV & isentropic potential vorticity & ISLSCP & International Satellite Land-Surface \\
\hline $\begin{array}{l}\text { IQSY } \\
\text { ir }\end{array}$ & $\begin{array}{l}\text { International Quiet Sun Years } \\
\text { infrared (preferred over "IR") }\end{array}$ & & $\begin{array}{l}\text { Climatology Project (GEWEX and } \\
\text { WCRP) }\end{array}$ \\
\hline $\mathbf{I R}$ & Infrared ("ir" is preferred) & ISO & International Standards \\
\hline IRAP & Interdisciplinary Panel on Climate & & Organization \\
\hline IRC & $\begin{array}{l}\text { Change } \\
\text { International Radiation }\end{array}$ & ISP & $\begin{array}{l}\text { International Society for } \\
\text { Photogrammetry }\end{array}$ \\
\hline & Commission & ISPRS & International Society for \\
\hline IRD & $\begin{array}{l}\text { Instituto de Radioprotecao e } \\
\text { Dosimetria (Brazil) }\end{array}$ & & $\begin{array}{l}\text { Photogrammetry and Remote } \\
\text { Sensing }\end{array}$ \\
\hline IRF & instantaneous radiative transfer & ISPs & Integrated Steel Plants \\
\hline IRGA & infrared gas analyzer & ISRIC & International Soil Reference and \\
\hline IRIS & $\begin{array}{l}\text { Incorporated Research Institution } \\
\text { for Seismology }\end{array}$ & ISRO & $\begin{array}{l}\text { Indian Space Research } \\
\text { Organization }\end{array}$ \\
\hline IRIS & $\begin{array}{l}\text { infrared interferometer } \\
\text { spectrometer }\end{array}$ & ISS & integrated sounding system \\
\hline
\end{tabular}


GLI IL G IYE ACLONYMS AND ABBREVIATIONS

ISSC

ISSP

ISSS

ISSMFE

IST

ISTAP

ISTP

ISU

ISWS

ISY

ITASE

ITB

ITB

ITC

ITCZ

ITEM

ITEX

ITIR

ITIR

ITOS

ITOY

ITPP

ITPR

ITRA

ITRC

ITRDB

ITSC
International Social Science

Council

ICSU Solar System Panel

International Society of Soil

Science

International Society of Soil

Mechanics and Foundation

Engineering

Instrument Support Terminal

International Space Technology

Assessment Program

International Solar Terrestrial

Physics

International Space University

Illinois State Water Survey

International Space Year (1992)

International Trans-Antarctic

Scientific Expedition (PAGES)

Bandung Institute of Technology

(Indonesia)

Intergovernmental TOGA Board

International Institute for

Aerospace Survey and Earth

Sciences

Intertropical Convergence Zone

International Technology

Environmental (data base)

Management (system)

International Tundra Experiment

(USMAB Directorate on High

Latitude Ecosystems)

infrared thermal imaging

radiometer (now ASTER)

intermediate and thermal infrared radiometer

Improved TIROS Operational

System

International Tropospheric Ozone

Year

International TOVS Processing

Package

infrared temperature profile

radiometer

intercomparison of transmittance

and radiance algorithms

Industrial Toxicology Research

Centre

International Tree-Ring Data Bank

International TOVS Study

Conference
ITU

ITU

IUAES

IUB

IUBMB

IUBS

IUCAF

IUCC

IUCN

IUCr

IUCRM

IUCS

IUE

IUFRO

IUGG

IUGS

IUHPS

IUNS

IUPAB

IUPAC

IUPAP

IUPHAR

IUPS integrated terrain unit

International Telecommunication

Union

International Union of

Anthropological and Ethnological

Sciences

International Union of

Biochemistry (ICSU)

International Union of

Biochemistry and Molecular

Biology

International Union of Biological

Sciences (ICSU)

Inter-Union Commission on

Frequency Allocation for Radio

Astronomy and Space Science

Information Unit on Climate

Change

International Union for the

Conservation of Nature and

Natural Resources (now World

Conservation Union) (Switzerland)

International Union of

Crystallography

Inter-Union Commission on Radio

Meteorology

Inter-Union Commission on

Spectroscopy

international ultraviolet explorer

International Union of Forestry

Research Organizations

(Secretariat, Schonbruan, Vienna)

International Union of Geodesy

and Geophysics (ICSU)

International Union of Geological

Sciences (ICSU)

International Union of the History

and Philosophy of Science

International Union of Nutritional

Sciences

International Union for Pure and

Applied Biophysics (ICSU)

International Union of Pure and

Applied Chemistry (ICSU)

International Union of Pure and

Applied Physics (ICSU)

International Union of

Pharmacology

International Union of

Physiological Sciences 
(-LIAL IHATE ACDONYMS ANI) ABDLEVIATIONS

IUPsyS International Union of

Psychological Science

IUR International Union of

Radioecologists

IUTAM International Union of Theoretical and Applied Mechanics (ICSU)

IVA intra vehicular activities

Instituto Venezuelan de

Investigaciones Cientificas

IVL Swedish Environmental Research Institute

IV\&V independent verification and validation

IWG Investigators Working Group

IWGDMGC Interagency Working Group on

Data Management for Global Change (U.S.)

IWRA International Water Resources Association

IWSA International Water Supply Association

IZMIRAN Institute of Terrestrial Magnetism, Radio Research, and the Ionosphere (Academy of Sciences) (Russian Federation; formerly U.S.S.R.) 


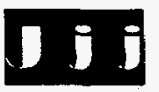

JASON Scientific panel convened by the MITRE Corporation

JAWS Joint Airport Weather Studies (project)

JECOR U.S.-Saudi Arabian Joint Commission of Economic Cooperation

JEM Japanese Experiment Module Japanese Earth Observing System Japanese Earth Observing Satellite Japanese Earth Remote-sensing Satellite-1

JERS Japanese Earth Resources Satellite (JPOP)

JGOFS Joint Global Ocean Flux Study

JGOFS (program) (IGBP and SCOR)

JGOFS PM JGOFS Photosynthetic Measurements (task team)

JGOFS-SSC JGOFS Scientific Steering Committee

JICST Japan Information Center of Science and Technology

JIU

JJA

JMA

JOC

JOI joint inspection unit June-July-August Japan Meteorological Agency Joint Organizing Committee Joint Oceanographic Institutions (Inc.)

JPL Jet Propulsion Laboratory (California Institute of Technology)

JPOP Japanese Polar-Orbiting Platform (JERS)

JSC Johnson Space Center

JSC Joint Scientific Committee (ICSU, WCRP, and WMO)

JSC-WCRP Joint Scientific Committee for the WCRP (ICSU and WMO)

JTC joint transfer correlation

JTU Jackson turbidity unit 


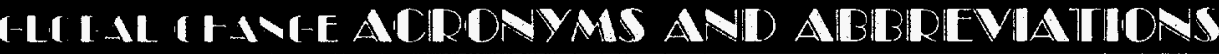

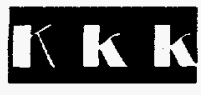

$\mathbf{K}$

kelvin, unit of thermodynamic

temperature (the number of kelvins equals the number of degrees

Celsius plus 273.15)

ka

thousands of years

KAHE

kbps

KEEP

time series station off Kahe Point, Hawaii

kilobits per second

Kuroshio Edge Exchange

Processes

KERFIX Kerguelen fixed station (France) (JGOFS)

$\mathrm{keV}$

kg

$\mathbf{k H z}$

kJ

km

$\mathrm{K}_{\mathrm{ow}}$

$\mathrm{kPa}$

$\mathbf{K r}$

KSC

kW

kilo electron volts

kilogram

kilohertz

kilojoule (energy measure)

kilometer

octanol-water partition coefficient pressure above ambient $(100 \mathrm{kPa}$

$=1$ atmosphere excess)

krypton

Kennedy Space Center (NASA)

kilowatt 


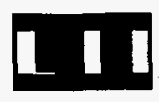

\section{U/C \\ LAC \\ LAC \\ LAF \\ LAGCM \\ LAGEOS \\ LAI \\ LAID}

LAMBADA

LAMP

LAN

Landsat

Landsat

LANL

LANL/ACL

LAPAN

LaRC

LASE

LASER

LASR

LASS

lat.

LAWS

LBL

LBL

LBLRTM

LCC

LCL

LDC land cover

levelized annual cost

local area coverage

Lake Acidification and Fisheries

(EPRI)

general circulation model at Los

Alamos National Laboratory

Laser Geodynamics Satellite

leaf area inclex

land-atmosphere interactions

dynamics

Large-scale Atmospheric Moisture

Balance of Amazona using Data

Assimilation (BAHC and GEWEX)

Lake Acidification Mitigation

Project (EPRI)

local area network

Land Remote-Sensing Satellite

land satellite (photographs of the

ground) (formerly ERTS) (NASA)

Los Alamos National Laboratory

Los Alamos National Laboratory's

Advanced Computing Laboratory

National Institute for Aeronautics

and Space (Indonesia)

Langley Research Center (NASA)

Lidar Atmospheric Sensing

Experiment

light amplification by stimulated

emission of radiation

Library Access, Search, and

Retrieval

Local Area Sounding System

latitude

laser atmospheric wind sounder

Lawrence Berkeley Laboratory

(now Lawrence Livermore

Laboratory) (University of

California)

line-by-line

line-by-line radiative transfer

model

land cover change

lifting condensation level

less-developed country
LDEO

LDGO

LDR

LEADEX

LEAPS

LEFI

LEMA

LERTS

LES

LFM

LI

Lidar

Lidar

LIDQA

LIGA

LIMS

LIPI

LIPI

LIPI

LIRAD

LIS

LIS

LITE

LLL

LLNL Lawrence Livermore National

LLW low-level waste

LMA Laboratoire de Meteorologie
Laboratory

Lamont-Doherty

Observatory

linear depolarization ratio

Leads Experiment

Species

local electric field instrument

Activity (GCTE)

Laboratoire d'Etudes et de

Recherches en Teledetection

Spatiale (France)

Lxporimental Satellite

library

laser-radar

light detection and ranging

(instrument)

Landsat Image Data Quality and

Analysis

(project)

Limb Infrared Monitor of the

Stratosphere

Marine Pollution Monitoring

Center (including LIPI's Network

of the Center for Research and

Development in Oceanography)

Minister of State for Population

environment

Sciences

lidar/radiometer

land information system

lightning imaging sensor

ar In-Space Technology

Experiment

Lawrence Livermore National 
LMD

LMER

LOICZ

LOICZ-SSC

long.

LORAN

LORCS

LOTREX

LOTREX

LPDA

LPDM

LPI

LPM

LR

LRA

LRP

LRPT

LSC

LSD

LSM

LSN

LST

LST

LTER

LTM

LTM

LTREB

LTRMP

LTRRS

LUCC

LUCC-CPPC

LVS

LW

LWC

LWIR
Laboratoire de Meteorologie

Dynamique

Land Margins Ecosystem Research

Land-Ocean Interactions in the

Coastal Zone (IGBP)

LOICZ Scientific Steering

Committee

longitude

Long-Range Navigation System

League of the Red Cross and Red

Crescent Societies

Land-Ocean Interactions in the

Coastal Zone (IGBP)

Longitudinal Land-Surface

Traverse Experiment

laser-phased Doppler anemometer

Lagrangian particle dispersion

model

low-pressure impactor

liters per minute

laser retroreflector

laser retroreflector array

Landscape Research Project (DOE)

low-resolution picture transmission

Lake Survey Center (NOAA)

least significant difference

LAWS Simulation Model

long- and short-normal resistivity

(geophysical logging)

local solar time

local standard time

Long-Term Ecological Research

(program) (NSF)

linear tangent model

long-term monitoring

Long-Term Research in

Environmental Biology

Long-Term Resource Monitoring

Program (U.S. Fish and Wildlife

Service)

Long-Term Regional Research Site

Land Use and Global Land-Cover

Change (IGBP and HDP)

LUCC Core Project Planning

Committee

low-volume sampler

longwave

liquid water content

long-wavelength (thermal) infrared
LWP

LWRM liquid water path

longwave radiation model 


\section{III III}

m

M-region

M\&CD

M\&E

MAA

MAB

MAC

MAC

MACDIF

MAGE

MAGE

MAGIC

MAGS

Magsat

MAHLOVS

MAKS

MAP

MAP3S

MAP3S/PCN

MAPS

MAPS

MAPS

MARC

MARDOS meter

Maunder region

Metals and Ceramics Division

monitoring and enforcement

moist-adiabatic adjustment

Man and the Biosphere

(programme) (UNESCO)

Middle Atmosphere Cooperation

(IGAC)

Multiphase Atmospheric Chemistry

(IGAC)

Mapping and Charting Data

Interchange Format (Canada)

Marine Aerosol and Gas Exchange

(IGAC)

Marine Gas Emissions,

Atmospheric Chemistry, and

Climate (IGAC)

Model of Acidification of

Groundwater in Catchment

Mackenzie GEWEX Study

(Canada)

Magnetic Field Satellite

Middle and High Latitudes

Oceanic Variability Study

Martin and Kitzis Sampler

(portable air sampler)

Middle Atmosphere Programme

Multistate Atmospheric Power

Production Pollution Study

Multistate Atmospheric Power

Production Pollution

Study/Precipitation Chemistry

Network

Map Analysis Package System

Measurement of Atmospheric

Pollution from Satellites

Mesoscale Analysis and Prediction

System

The Middle Atmosphere Responses

to Changes

IAEA CRP on "Sources of

Radioactivity in the Marine

Environment and Their Relative

Contributors to Overall Dose

Assessment from Marine

Radioactivity"
MARECO

MARIA

MASC

MASFLEX

MAST

MATS

MAW

MB

mb

Mbps

MBL

MBT

MCC

$\mathrm{MCC}$

McIDAS

MCL

mom

MCP

MCS

MCS

MCSST

MCZ

MDA

MDC

MDR

MDSRS

MECCA

MED

MEDCOM
Marine Radioecology Working

Group (IUR)

Methods for Assessing the

Radiological Impact of Accidents

(CEC)

Mountain Administration Support

Center (NOAA)

Marginal Sea Flux Experiment (in the West Pacific) (IGOPS)

Marine Science and Technology

Programme (CEC)

Mesoscale Atmospheric Transport

Studies

Ministry of Agriculture and Water

(Saudi Arabia)

Manitoba

millibar

megabits per second

marine boundary layer

Mechanical Bathythermograph

mesoscale convective complex

Mission Control Center

Man-computer Interactive Data

Access System

maximum contaminant level

million cubic metre

Meteorological Communications

Package

Mesoscale Cloud System

Mesoscale Convective Systems

Multi-Channel Sea Surface

Temperature (algorithm)

Mesoscale Compressible

Community Model

Methodology of Decision Analysis (project)

more developed country

minimum detectable radiance

morphology-dependent stimulated

Raman scattering

Model Evaluation Consortium for

Climate Assessment

Mediterranean (the region)

(START)

Mediterranean Planning Committee

(START) 
T-LI IL IIAY-E ACDONYMS ANID AIBBLEVIATIONS

\begin{tabular}{|c|c|c|c|}
\hline MEDCOM & $\begin{array}{l}\text { Mediterranean Regional } \\
\text { Committee (START) }\end{array}$ & MFRSR & $\begin{array}{l}\text { multifilter rotating shadowband } \\
\text { radiometer }\end{array}$ \\
\hline MEDIAS & $\begin{array}{l}\text { Mediterranean and Subtropical } \\
\text { Africa }\end{array}$ & MGE & $\begin{array}{l}\text { MicroStation Foundation and } \\
\text { Modular GIS Environment (by }\end{array}$ \\
\hline MEDIAS & $\begin{array}{l}\text { Mediterranean Basin and Sub- } \\
\text { tropical Africa (regional research } \\
\text { network) }\end{array}$ & $\begin{array}{l}\text { Mha } \\
\text { MHRS }\end{array}$ & $\begin{array}{l}\text { intergraph) } \\
\text { million hectare } \\
\text { Modified Hazard Ranking System }\end{array}$ \\
\hline MEDIAS & $\begin{array}{l}\text { Reseau de Recherche Regionale } \\
\text { pour le Bassin Mediterraneen et } \\
\text { l'Afrique Subtropicale }\end{array}$ & MHS & $\begin{array}{l}\text { (DOE) } \\
\text { Message Handling System } \\
\text { (electronic) }\end{array}$ \\
\hline MEHALICE & $\begin{array}{l}\text { Methane and Halocarbons } \\
\text { Intercalibrations Experiment } \\
\text { (IGAC) } \\
\text { medium expendable launch vehicle }\end{array}$ & $\begin{array}{l}\text { MHS } \\
\text { MHz } \\
\text { MIADS }\end{array}$ & $\begin{array}{l}\text { microwave humidity sounder } \\
\text { megahertz } \\
\text { map information assembly and } \\
\text { display system }\end{array}$ \\
\hline MEPED & $\begin{array}{l}\text { medium energy proton and electron } \\
\text { detector }\end{array}$ & $\begin{array}{l}\text { MIBK } \\
\text { MILOX }\end{array}$ & $\begin{array}{l}\text { methyl isobutyl ketone } \\
\text { Mid-Latitude Ecosystems and }\end{array}$ \\
\hline MERIS & $\begin{array}{l}\text { medium-resolution imaging } \\
\text { spectrometer }\end{array}$ & MIMD & $\begin{array}{l}\text { Photochemical Oxidants (IGAC) } \\
\text { multiple instruction, multiple data }\end{array}$ \\
\hline MERIT & $\begin{array}{l}\text { Monitoring Earth's Rotation and } \\
\text { Intercomparison of Techniques }\end{array}$ & MIMR & $\begin{array}{l}\text { (system) } \\
\text { multifrequency imaging microwave }\end{array}$ \\
\hline MERS & $\begin{array}{l}\text { Medium Resolution Imaging } \\
\text { Spectrometer }\end{array}$ & MIMR & $\begin{array}{l}\text { radiometer (formerly HIMSS) } \\
\text { multiband/multifrequency imaging }\end{array}$ \\
\hline MES & Military Engineering Services & & microwave radiometer \\
\hline MESOSOFT & $\begin{array}{l}\text { Mesoscale Data Management } \\
\text { Facility }\end{array}$ & $\begin{array}{l}\text { MINK } \\
\text { MIPAS }\end{array}$ & $\begin{array}{l}\text { Missouri-Iowa-Nebraska-Kansas } \\
\text { Michelson interferometer for }\end{array}$ \\
\hline MESEEC & $\begin{array}{l}\text { Methodologies to Estimate Social, } \\
\text { Environmental, and Economic }\end{array}$ & 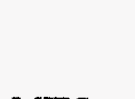 & $\begin{array}{l}\text { passive atmospheric sounding } \\
\text { (sounder) }\end{array}$ \\
\hline MESSR & $\begin{array}{l}\text { Consequences } \\
\text { multispectral electronic } \\
\text { self-scanning radiometer }\end{array}$ & $\begin{array}{l}\text { MIPS } \\
\text { MIRSL }\end{array}$ & $\begin{array}{l}\text { millions of instructions per second } \\
\text { Microwave Remote Sensing } \\
\text { Laboratory }\end{array}$ \\
\hline METEOR & $\begin{array}{l}\text { Operational Weather Satellite } \\
\text { (Russian Federation; formerly } \\
\text { U.S.S.R.) }\end{array}$ & $\begin{array}{l}\text { MIS } \\
\text { MISR }\end{array}$ & $\begin{array}{l}\text { management information system } \\
\text { multi-angle imaging spectro- } \\
\text { radiometer }\end{array}$ \\
\hline METEOSAT & $\begin{array}{l}\text { Geosynchronous Meteorology } \\
\text { Satellite (ESA) }\end{array}$ & MIT & $\begin{array}{l}\text { Massachusetts Institute of } \\
\text { Technology (U.S.) }\end{array}$ \\
\hline METEOSAT & $\begin{array}{l}\text { Meteorological Satellite } \\
\text { (EUMETSAT) }\end{array}$ & MITI & $\begin{array}{l}\text { Ministry of International Trade and } \\
\text { Industry (Japan) }\end{array}$ \\
\hline METOP & $\begin{array}{l}\text { Meteorological Operational } \\
\text { Satellite }\end{array}$ & MITRE & $\begin{array}{l}\text { Massachusetts Institute of } \\
\text { Technology Research }\end{array}$ \\
\hline $\mathrm{MeV}$ & mega electron volts & & Establishment \\
\hline MFE & Magnetic Fusion Energy (program) & MITRE & Miniature Individual Transmitter \\
\hline $\begin{array}{l}\text { MFE/ } \\
\text { MAGNOLIA }\end{array}$ & MFE/Magnetic Field Experiment & MIZ & $\begin{array}{l}\text { Receiver Equipment } \\
\text { Marginal Ice Zone }\end{array}$ \\
\hline Mflops & $\begin{array}{l}\text { One megaflop equals } 1 \text { million } \\
\left(10^{6}\right) \text { floating point operations per } \\
\text { second } \\
\text { mixed-function oxidase (enzyme } \\
\text { system) }\end{array}$ & $\begin{array}{l}\text { MIZEX } \\
\text { MJ } \\
\text { mks }\end{array}$ & $\begin{array}{l}\text { Marginal Ice Zone Experiment } \\
\text { megajoule } \\
\text { meter-kilogram-second } \\
\text { (measurement system; replaced by } \\
\text { SI) (preferred over "MKS") }\end{array}$ \\
\hline
\end{tabular}




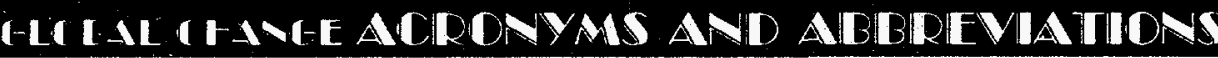

MKS

mksa

MKSA

MLE

MLO

MLOPEX II

MLR

MLS

mm

MM4

MM5

MMAD

MM-Al

MMES

MMI

mmscmd

MMTS

MNRT

MOA

MOBILHY

MOC

MODECS

MODIS

MODIS-N

MODIS-T

MODMAP

MOLA meter-kilogram-second

(measurement system; replaced by

SI) ("mksa" is preferred)

meter-kilogram-second-ampere

(measurement system; replaced by

SI) (preferred over "MKSA")

meter-kilogram-second-ampere

(measurement system; replaced by

SI) ("mksa" is preferred)

maximum likelihood estimation

Mauna Loa Observatory (Hawaii)

(CMDL)

Mauna Loa Observatory

Photochemical Experiment II

multiple linear regression

microwave limb sounder

millimeter

mesoscale model 4 (Pennsylvania

State/National Center for

Atmospheric Research)

Mesoscale model 5 (Pennsylvania

State/National Center for

Atmospheric Research)

mass median aerodynamic

diameter

total monomeric aluminum

Martin Marietta Energy Systems,

Inc. ("Energy Systems" is

preferred)

Modified Mercalli Intensities

million metric standard cubic metre

per day

Maximum-Minimum Temperature

System

Minister of State for Research and

Technology

Memorandum of Agreement

Mobilisation du Bilan Hydrique

Memorandum of Cooperation

molecular design of chemical

systems

moderate-resolution imaging

spectroradiometer

moderate-resolution imaging

spectrometer-Nadir

moderate-resolution imaging

spectrometer-tilt

modernization of mapping

techniques

Mars Observer Laser Altimeter
MOM

MOM

MOMS

MONEX

MONSEE

MOP

MOPITT

MORACC

MORFLOT

MORS

MOS-1

MOS

MOS

MOSS

MOT

MOU

MPC

MPC

MPE

MPF

MPI

MPL

MPL

MR

MRI

MRIR

MS

MSA

MSC

MSC

MSFC

MSL

MSP

MSR

MSS

MSS

MSU
Mission Operations Manager

modular ocean model

modular optoelectronic

multispectral scanner

Monsoon Experiment

Monitoring of the Sun-Earth

Environment

Meteosat Operational Programme

Measurements of Pollution in the

Troposphere

modeling radiation clouds, and

convection

Ministry of the Merchant Marine

of the Soviet Union (Russian

Federation; formerly U.S.S.R.)

Group of Experts Monitoring of

Radioactive Substances in the

Baltic Sea

Marine Observation Satellite-1

(Japan)

Marine Observation Satellite

model output statistics

Map Overlay Statistical System (by

Autometrics)

Ministry of Transport (Cangda)

Memorandum of Understanding

massively parallel computer

maximum permissible

concentration

Mission to Planet Earth

momentary power failure

Max Planck Institute (Germany)

Marine Physical Laboratory

Micropulse Lidar

Microwave Radiometer

Meteorology Research, Inc.

medium resolution infrared

radiometer

mass spectrometry

methanesulfonate

Marine Sciences Council

Meteorological Satellite Center

Marshall Space Flight Center

mean sea level

Motion-Sensing Platform

microwave scanning radiometer

multispectral scanner (Landsat)

multispectral scanner system

microwave sounding unit 


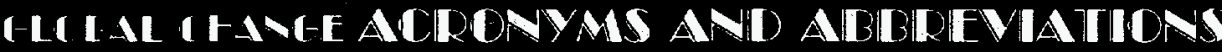

MST

MT

MTCE

MT-CLIM

MTCR

MTMC

MTOE

MTS

MU

MUID

MUIR

MVS

mW

MW

MWDI

MWIR

MWL

MWR

MWSR
Ministry of Environment and Science

million tonne

million tonnes of carbon equivalent Mountain Climate Model million tonnes of coal replacement Military Traffic Management Command million tonnes of oil equivalent microwave temperature sounder microwave unit

map unit identification data map unit interpretation record Minimum-Variance-Simultaneous milliwatt megawatt Master Water Data Index medium-wavelength infrared municipal waste leachate microwave radiometer microwave water substance radiometer 


\section{II}

\begin{tabular}{|c|c|}
\hline NA & North America(n) \\
\hline $\mathrm{N}_{2} \mathrm{O}$ & nitrous oxide \\
\hline $\mathrm{N}_{2} \mathrm{O}_{5}$ & $\begin{array}{l}\text { nitrogen pentoxide, nitric } \\
\text { anhydride }\end{array}$ \\
\hline NAAQ & National Ambient Air Quality \\
\hline NAAQS & $\begin{array}{l}\text { National Ambient Air Quality } \\
\text { Standards }\end{array}$ \\
\hline NABE & $\begin{array}{l}\text { North Atlantic Bloom Experiment } \\
\text { (JGOFS and IGAC) }\end{array}$ \\
\hline NAD 27 & North American Datum of 1927 \\
\hline NAD 83 & North American Datum of 1983 \\
\hline NADP & $\begin{array}{l}\text { National Atmospheric Deposition } \\
\text { Program (EPA) }\end{array}$ \\
\hline NADP/NTN & $\begin{array}{l}\text { National Atmospheric Deposition } \\
\text { Program/National Trends Network }\end{array}$ \\
\hline NADW & North Atlantic deep water \\
\hline NAEG & Nevada Applied Ecology Group \\
\hline NAF & Northern Africa (START) \\
\hline NAFCOM & $\begin{array}{l}\text { Northern Africa Committee } \\
\text { (START) }\end{array}$ \\
\hline NAIC & Newly Agro-Industrialized Country \\
\hline NAL & National Agricultural Library \\
\hline NALMS & $\begin{array}{l}\text { North American Lake Management } \\
\text { Society }\end{array}$ \\
\hline NAMAS & $\begin{array}{l}\text { National Measurement } \\
\text { Accreditation Service }\end{array}$ \\
\hline NAPAP & $\begin{array}{l}\text { National Acid Precipitation } \\
\text { Assessment Program }\end{array}$ \\
\hline NAR & net assimilation rate \\
\hline NARA & $\begin{array}{l}\text { National Archives and Records } \\
\text { Administration }\end{array}$ \\
\hline NARC & $\begin{array}{l}\text { National Atmospheric Radiation } \\
\text { Centre (AES) }\end{array}$ \\
\hline NARE & $\begin{array}{l}\text { North Atlantic Regional } \\
\text { Experiment (IGAC) }\end{array}$ \\
\hline NARL & $\begin{array}{l}\text { National Arctic Research } \\
\text { Laboratory (Barrow, Alaska) }\end{array}$ \\
\hline NARS & $\begin{array}{l}\text { National Archives and Records } \\
\text { Service (GSA) }\end{array}$ \\
\hline NARSNDF & $\begin{array}{l}\text { North Atlantic Regional Study } \\
\text { Narrative Data File }\end{array}$ \\
\hline NAS & $\begin{array}{l}\text { National Academy of Sciences } \\
\text { (U.S.) }\end{array}$ \\
\hline NASA & $\begin{array}{l}\text { National Aeronautics and Space } \\
\text { Administration (U.S.) }\end{array}$ \\
\hline & NASA/Environmental \\
\hline
\end{tabular}

\begin{tabular}{|c|c|}
\hline \multirow[t]{2}{*}{ NASC } & North American Stratigraphic \\
\hline & Code \\
\hline NASCOM & NASA Communications Network \\
\hline NASDA & National Space Development \\
\hline & Agency (Japan) \\
\hline NASM & National Air and Space Museum \\
\hline NASQUAN & $\begin{array}{l}\text { National Stream Water Quality } \\
\text { Accounting Network }\end{array}$ \\
\hline NAST & $\begin{array}{l}\text { National Academy of Science and } \\
\text { Technology }\end{array}$ \\
\hline NATO & North Atlantic Treaty Organization \\
\hline NATT & $\begin{array}{l}\text { Northern Australian Tropical } \\
\text { Transect }\end{array}$ \\
\hline NAVSTAR & $\begin{array}{l}\text { Navigation Satellite Timing and } \\
\text { Ranging }\end{array}$ \\
\hline Navstar & $\begin{array}{l}\text { Global Positioning Satellite } \\
\text { Communication Set }\end{array}$ \\
\hline NAVSWC & $\begin{array}{l}\text { Naval Surface Warfare Center } \\
\text { (DOD) }\end{array}$ \\
\hline NAWDEX & $\begin{array}{l}\text { National Water Data Exchange } \\
\text { (USGS) }\end{array}$ \\
\hline NBIOME & $\begin{array}{l}\text { Northern Biosphere Observation } \\
\text { and Modeling Experiment }\end{array}$ \\
\hline NBIS & $\begin{array}{l}\text { Northern Biosphere Information } \\
\text { System }\end{array}$ \\
\hline NBRF & $\begin{array}{l}\text { National Biomedical Research } \\
\text { Foundation }\end{array}$ \\
\hline NBRI & $\begin{array}{l}\text { National Botanical Research } \\
\text { Institute }\end{array}$ \\
\hline NBS & National Biological Survey \\
\hline NBS & $\begin{array}{l}\text { National Bureau of Standards (now } \\
\text { NIST) }\end{array}$ \\
\hline NC & National Committee (IGBP) \\
\hline NCAER & $\begin{array}{l}\text { National Council of Applied } \\
\text { Economic Research }\end{array}$ \\
\hline NCAR & $\begin{array}{l}\text { National Center for Atmospheric } \\
\text { Research (NOAA) (Boulder, } \\
\text { Colo.) }\end{array}$ \\
\hline NCASI & $\begin{array}{l}\text { National Council on Air and } \\
\text { Stream Improvement }\end{array}$ \\
\hline NCC & $\begin{array}{l}\text { National Climatic Center (now } \\
\text { NCDC) }\end{array}$ \\
\hline $\mathrm{NCC}$ & Network Control Center \\
\hline NCDC & $\begin{array}{l}\text { National Climatic Data Center } \\
\text { (Asheville, N.C.) (NOAA) }\end{array}$ \\
\hline
\end{tabular}




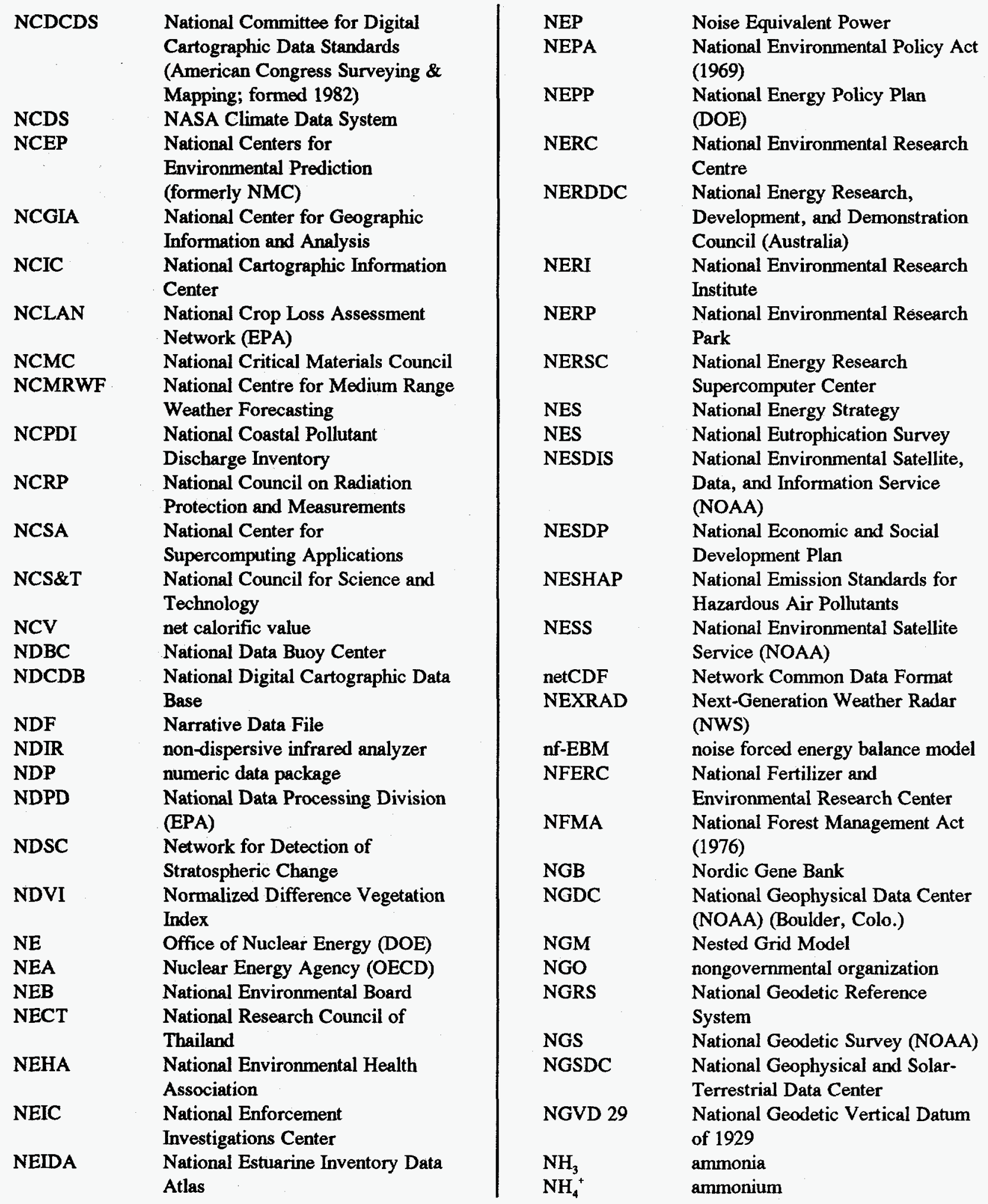




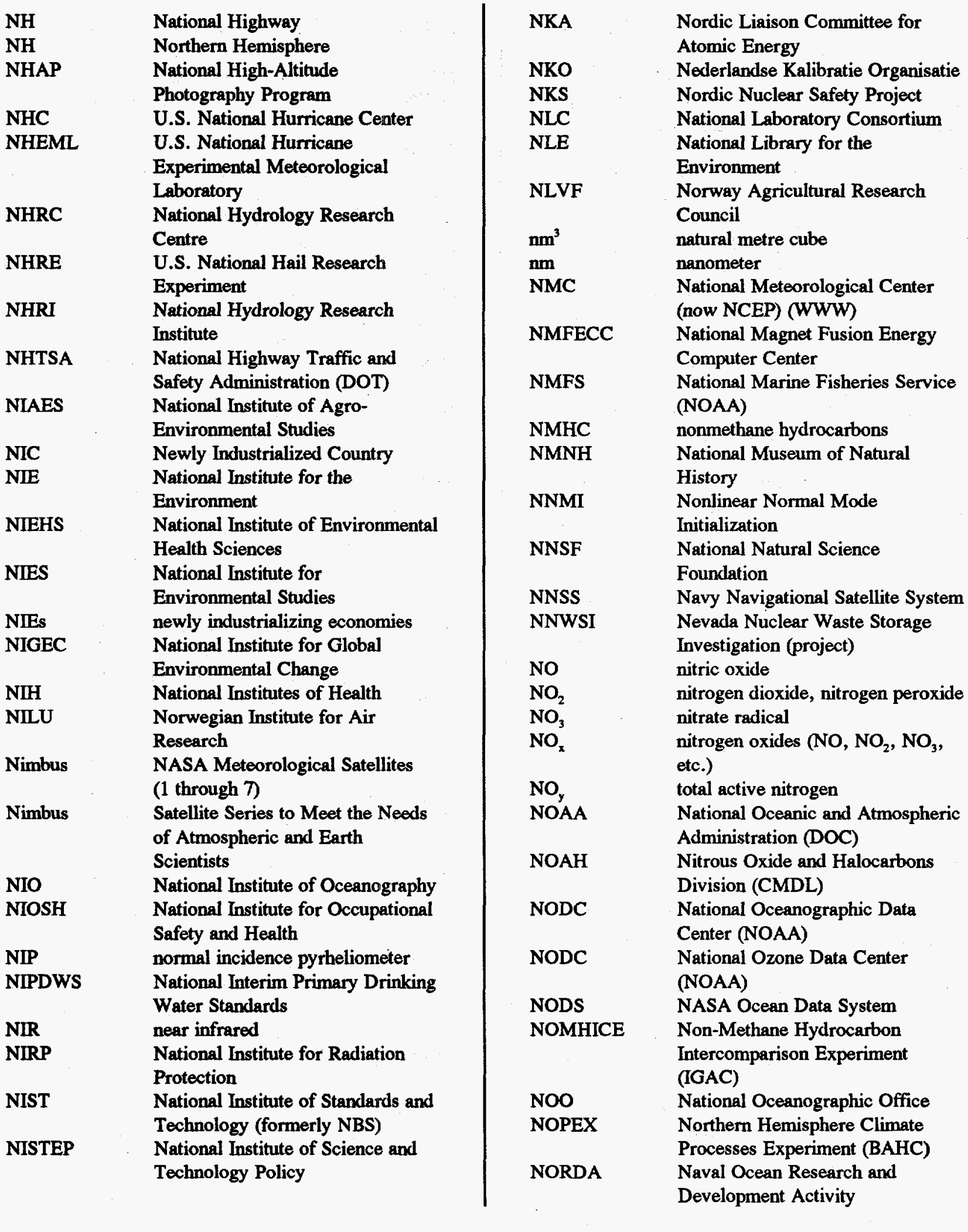


GLC IL II AYTE ACDONYMS AND ABBRIEVIATIDNS

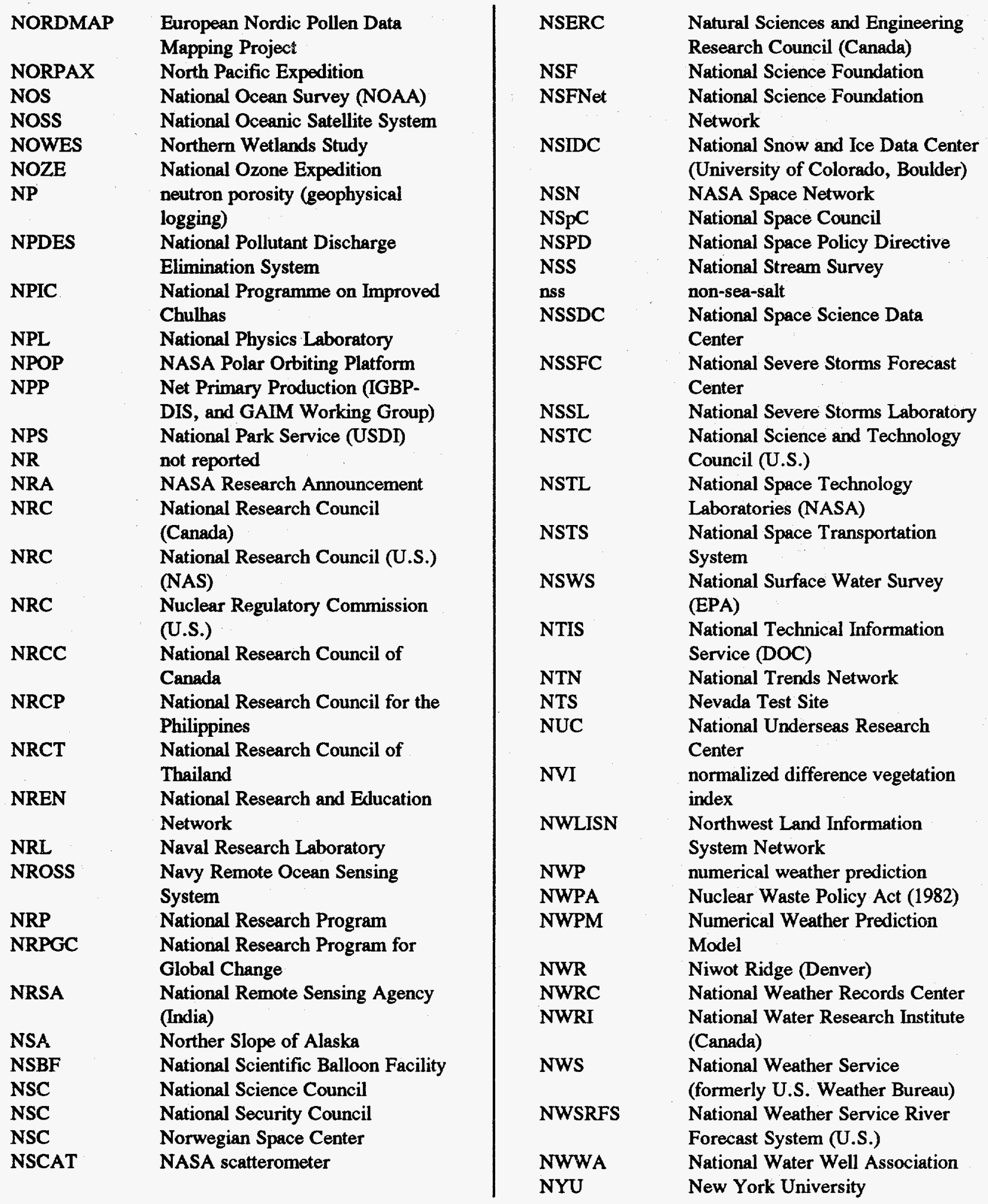

Natural Sciences and Engineering

National Science Foundation

National Snow and Ice Data Center

(University of Colorado, Boulder)

National Space Council

National Space Policy Directive

National Stream Survey

National Space Science Data

Center

National Severe Storms Laboratory

National Science and Technology

Laboratories (NASA)

System

National Surface Water Survey

Service (DOC)

National Trends Network

Center

Council of

National Research and Education

Navy Remote Ocean Sensing

System

National Research Program for

Global Change

(India)

Norther Slope of Alaska

National Scientific Balloon Facilit

National Science Council

National Security Council

Norwegian Space Cente

NYU

New York University 


\section{HIIA IFATEE ACLONYMS AND AMBBIEVIAIIONS}

$\begin{array}{ll}\text { NYU/Ultra } & \text { New York University's } \\ & \text { Ultracomputer (project) } \\ \text { NZOI } & \text { New Zealand Oceanographic } \\ \text { NZP } & \text { Institute } \\ \text { National Zoological Park }\end{array}$


TLLAL I FAYE ACDONYMS AND ABBREVIATIIONS

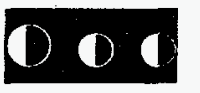

$\mathrm{O} / \mathrm{N}$
$\mathrm{O}_{2}$
$\mathrm{O}_{3}$
$\mathrm{O}_{\mathrm{x}}$
$\mathrm{OAR}$

OARM

OAS

OASIS

OAST

OAU

OAXTC

OCAM

OCC

OCE

OCTS

ODA

ODAS

ODB

ODC

ODP

ODP

OEC

OECD

OEG

OEP

OER

OES

OFDA

OFS

OGC

OGCM oxygen-to-nitrogen ratio

(normal) molecular oxygen

ozone

odd oxygen $\left(\mathrm{O}+\mathrm{O}_{3}\right)$

Office of Atmospheric Research (NOAA)

Office of Administration and

Resources Management

Organization of American States

Observation at Several Interacting

Scales

Office of Aeronautics Space

Technology (NASA)

Organization of African Unity

Ocean Atmosphere Exchange of

Trace Compounds

Afro-Malagasy Common

Organization

Operations Control Center

Oceania (START)

ocean color and temperature scanner

Overseas Development Agency

(U.K.)

Oceanic Data Assimilation System

Orbit Determination Beacon

other data center

Ocean Drilling Program

ozone-depleting potential

Office of Environmental

Compliance

Organization for Economic

Cooperation and Development

Office of Environmental Guidance

Office of Economic Planning

Office of Energy Research (DOE)

Bureau of Oceans, International

Environment, and Scientific

Affairs (USDA)

Office of U.S. Foreign Disaster

Assistance

Office of the Foreign Secretary

(NAS and NRC)

Oregon Graduate Center

(Beaverton, Oreg.)

ocean general circulation model
OGIST

OGWP

$\mathrm{OH}$

OHER

OHF

OHP

OIEA

OIRM

OIS

OKCS

OLIPAC

OLR

OLS

OLS

OLS

OM-Al

OMB

OMCS

OMDR

OMEX

OMP

OMWOG

ONEB

ONR

ONWI

OPAC

OPC

OPEC

OPEX

OPM
Oregon Graduate Institute of

Science and Technology

Office of Ground Water Protection

(EPA)

hydroxyl radical

Office of Health and

Environmental Research (DOE)

Old Hydrofracture Facility (pond)

Operational Hydrology Programme

(WMO)

Office of Integrated Environmental

Analysis (EPA)

Office of Information Resources

Management (EPA)

Operational Information Service

(WWW)

Oklahoma Climatological Survey

Oligotrophic Pacific (France)

(JGOFS)

outgoing long-wave radiation

operational license stage

operational line scanner

optical line scanner

organic monomeric aluminum

Office of Management and Budget

(Independent)

ozone monitor comparison system

Optical Memory Disk Recorder

Animation System

Ocean Margin Exchanges

Ocean Margins Program

Ocean-based Measurements

Working Group

Office of National Environmental

Board

Office of Naval Research (U.S.)

Office of Nuclear Waste Isolation

Online Public Access Catalog

ordinary portland cement

Organization of Petroleum

Exporting Countries

Operational and Executive

Personnel for Civil Service

Office of Personnel Management 
OPPE

OPS

OPT

ORAU

ORD

ORISE

ORNL

ORNL/ACL

ORR

ORSTOM

OSB

OSC

OSC

OSHA

OSHA

OSR

OSSA

OSSE

OST

OSTI

OSTP

OSU

OSWER

OTA

OTEC

OTS

OWDC

OWRT

OWSE

OWSE-NA
Office of Policy, Planning, and Evaluation (EPA) optical sensor Optimization Under Uncertainty Project

Oak Ridge Associated Universities Office of Research \& Development (EPA)

Oak Ridge Institute for Science Education

Oak Ridge National Laboratory

ORNL/Advanced Computing

Laboratory

Oak Ridge Reservation

Office de la Recherche Scientifique et Technique d'Outre-mer (Office of Overseas Scientific and Technical Research) (France) Ocean Studies Board Office of Scientific Computing (DOE)

Orbital Sciences Corporation Occupational Safety and Health Act (1970)

Occupational Safety and Health Administration

Office of Sponsored Research Office of Space Science and Applications (NASA HQ) Observing System Simulation Experiments

Office of Science and Technology Office of Scientific and Technical Information (DOE)

Office of Science and Technology Policy

Oregon State University

Office of Solid Waste and Energy Response (EPA)

Office of Technology Assessment ocean thermal energy conversion Office of Toxic Substances (EPA) Office of Water Data Coordination (USGS)

Office of Water Research and Technology (DOI)

Operational WWW Systems Evaluation

Operational WWW Systems

Evaluation-North America 


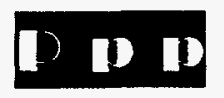

P/R

P-Code

PAAD

PAC

PACER

PAD

PAGES

PAGES-

EXCOMM

PAGES-SSC

PAH

PAHO

PALE

PAM

PAN

PANASH

PAR

PAR

PAR

PARCC

PASA

PASC

PASE

PBL

PBM

PBMR

PBU

PC

PCA

PCAST

PCB

PCMDI

${ }_{\mathrm{PCU}}^{\mathrm{CO}_{2}}$ productivity/respiration

GPS precision transmission code

Program Assistance Approval

Document

Polar Atmospheric Chemistry

Program for the Acceleration of

Commercial Energy Research

Program Approval Document

Past Global Changes (IGBP)

PAGES Executive Committee

PAGES Scientific Steering

Committee

polycyclic aromatic hydrocarbon

Pan American Health Organization

Palaeoclimate from Arctic Lakes and Estuaries (PAGES)

Portable Automated Mesonet

peroxyacetylnitrate

Paleoclimates of the Northern and

Southern Hemisphere (PAGES)

photosynthetically active radiation

photosynthetically available

radiation

Project Appraisal Report

precision, accuracy,

representativeness, completeness, and comparability

Participating Agency Service

Agreement

Polar Atmospheric and Snow

Chemistry (IGAC)

Polar Air-Snow Experiment

planetary boundary layer

peribacteroid membrane

Pushbroom Microwave Radiometer

peribacteroid unit

personal computer

principal components analysis

President's Council of Advisors on

Science and Technology

polychlorinated biphenyl

Program for Climate Model

Diagnosis and Intercomparison

partial pressure of $\mathrm{CO}_{2}$

platinum cobalt unit
PCR

PCSSD

PDA

PDB

PDB

PDE

pdf

PDL

PDRCA

PDS

PDS

PDSI

PECO

PEM

PEMD

PEP

PEPCase

PER

PERD

PFCs

PFRA

PFTs

$\mathrm{Pg}$

PG

PGC

PGS

PHOXA

PHSA

PI

PIC

PIC

PICES polymerase chain technology

Philippines Council for Sustainable

Development

phased-Doppler anemometer

isotope standard for carbon

Pee Dee Belemnite (Pee Dee

River, S.C.)

partial differential equation

probability density function

polarization-diversity lidar

Participatory Development

Resource Centre for Africa

Planetary Data System

photothermal deflection

spectroscopy

Palmer Drought Severity Index

CEC-COPERNICUS Program

Particle Environment Monitor

personalized educational materials

development

Pole-Equator-Pole (PAGES)

(Palaeoclimates Initiative for the

Western Hemisphere)

Phosphenol-pyruvate carboxylase

Program for Ecological Research

Panel for Energy Research and

Development

perfluorocarbon compounds

Prairie Farm Rehabilitation

Administration

perfluorocarbon tracers

pentagram

planning group

Program Grande Carajas (Brazil)

Product Generation System

photochemical oxidant and acid

deposition model application within

the framework of control strategy

development

Public Health Service Act (1965)

principal investigator

particle in cell

particulate inorganic carbon

North Pacific Marine Science

Organization 


\begin{tabular}{|c|c|}
\hline PIL.PS & $\begin{array}{l}\text { Project for Intercomparison of } \\
\text { Land-surface Parameterization } \\
\text { Schemes (GCIP/GEWEX) }\end{array}$ \\
\hline PIO & $\begin{array}{l}\text { Project Implementation Order } \\
\text { (AID) }\end{array}$ \\
\hline $\mathrm{PIO} / \mathrm{C}$ & PIO for Commodities \\
\hline $\mathrm{PIO} / \mathrm{P}$ & PIO for Participant Training \\
\hline $\mathrm{PIO} / \mathrm{T}$ & $\begin{array}{l}\text { PIO for Contract Technicians or } \\
\text { Contract Services }\end{array}$ \\
\hline PIP & $\begin{array}{l}\text { Performance Improvement } \\
\text { Program }\end{array}$ \\
\hline PIP & Project Implementation Plan (AID) \\
\hline PIP & $\begin{array}{l}\text { WetNet Precipitation (algorithm) } \\
\text { Intercomparison Project }\end{array}$ \\
\hline PIR & precision infrared radiometer \\
\hline PIRLA & $\begin{array}{l}\text { Paleoecological Reconstruction of } \\
\text { Recent Lake Acidification (EPRI) }\end{array}$ \\
\hline PJ & petajoule \\
\hline PL & public law \\
\hline PLDS & Planetary Land Data System \\
\hline PLDS & Pilot Land Data System \\
\hline PM & $\begin{array}{l}\text { photosynthetic measurements } \\
\text { (JGOFS) }\end{array}$ \\
\hline PM-10 & $\begin{array}{l}\text { particulate matter less than } \\
10 \text { micrometers }\end{array}$ \\
\hline PMC & pollen mother cell \\
\hline PMC & Pressure-Modulator Cell \\
\hline PMEL & $\begin{array}{l}\text { Pacific Marine Environmental } \\
\text { Laboratory (NOAA and ERL) }\end{array}$ \\
\hline PMF & probable maximum flood \\
\hline PMIP & $\begin{array}{l}\text { Paleoclimate Modeling } \\
\text { Intercomparison Project (NATO } \\
\text { and PAGES) }\end{array}$ \\
\hline PMIR & $\begin{array}{l}\text { Pressure Modulator Infrared } \\
\text { Radiometer }\end{array}$ \\
\hline PMOD & $\begin{array}{l}\text { Physikalisch-Meteorologisches } \\
\text { Observatorium Davos (WRC) }\end{array}$ \\
\hline PMP & probable maximum precipitation \\
\hline PMR & pressure-modulated radiometer \\
\hline PNEDC & $\begin{array}{l}\text { Programme Nationale d'Etude de } \\
\text { la Dynamique du Climat (France) }\end{array}$ \\
\hline PNL & Pacific Northwest Laboratory \\
\hline $\begin{array}{l}\mathrm{PO}_{4} \\
\mathrm{POC}\end{array}$ & $\begin{array}{l}\text { phosphate } \\
\text { particulate organic carbon }\end{array}$ \\
\hline POCC & Payload Operations Control Center \\
\hline POCM & parallel ocean-climate model \\
\hline $\begin{array}{l}\text { POD } \\
\text { POEM }\end{array}$ & $\begin{array}{l}\text { Precision Orbit Determination } \\
\text { polar-orbiting earth observation }\end{array}$ \\
\hline
\end{tabular}

\begin{tabular}{|c|c|}
\hline POEMS & $\begin{array}{l}\text { positron electron magnetic } \\
\text { spectrometer }\end{array}$ \\
\hline POES & $\begin{array}{l}\text { Polar-Orbiting Environmental } \\
\text { Satellite }\end{array}$ \\
\hline POES & $\begin{array}{l}\text { Polar-Orbiting Operational } \\
\text { Environmental Satellite }\end{array}$ \\
\hline POGO & $\begin{array}{l}\text { Polar-Orbiting Geophysical } \\
\text { Observatory }\end{array}$ \\
\hline POLDER & $\begin{array}{l}\text { Polarization and Directionality of } \\
\text { the Earth's Reflectances }\end{array}$ \\
\hline POLES & polar exchange at the sea surface \\
\hline POM & particulate organic matter \\
\hline POM & polycyclic organic matter \\
\hline PON & particulate organic nitrogen \\
\hline POOZ & Permanently Open Ocean Zone \\
\hline PP & perfect program (approach) \\
\hline ppb & parts per billion \\
\hline ppbv & parts per $10^{9}$ by volume \\
\hline PPFD & photosynthetic photon flux density \\
\hline ppm & parts per million \\
\hline ppmv & parts per $10^{6}$ by volume \\
\hline PPP & Polluter pays principle \\
\hline PPR & photopolarimeter radiometer \\
\hline ppt & parts per trillion \\
\hline pptv & parts per $10^{12}$ by volume \\
\hline PR & precipitation radar \\
\hline PRARE & $\begin{array}{l}\text { precise range and range-rate } \\
\text { equipment }\end{array}$ \\
\hline PRAREE & $\begin{array}{l}\text { precise range and range rate } \\
\text { equipment-extended }\end{array}$ \\
\hline PRC & People's Republic of China \\
\hline PRC & precipitation \\
\hline PRF & pulse repetition frequency \\
\hline PRL & Physical Research Laboratory \\
\hline PRN & Pseudo-Random Noise \\
\hline PROBE & $\begin{array}{l}\text { Pilot Radiation Observation } \\
\text { Experiment }\end{array}$ \\
\hline PRT & precision radiation thermometer \\
\hline PSA & Pacific Sciences Association \\
\hline PSAC & $\begin{array}{l}\text { President's Science Advisory } \\
\text { Committee }\end{array}$ \\
\hline PSC & polar stratospheric clouds \\
\hline PSD & $\begin{array}{l}\text { prevention of significant } \\
\text { deterioration (NPS, regulations of } \\
\text { the CAA) }\end{array}$ \\
\hline PSDPC & $\begin{array}{l}\text { Polar Satellite Data Processing } \\
\text { Centre }\end{array}$ \\
\hline Psfc & surface pressure \\
\hline PSFG & $\begin{array}{l}\text { Permanent Service on the } \\
\text { Fluctuation of Glaciers }\end{array}$ \\
\hline
\end{tabular}




$\begin{array}{ll}\text { PSL } & \text { polystyrene latex } \\ \text { PSO } & \text { Project Science Office } \\ \text { PSPDC } & \text { Polar Satellite Precipitation Data } \\ & \text { Centre } \\ \text { PSSI } & \text { Pacific/Sulfur/Stratus Investigation } \\ \text { PSU } & \text { Pennsylvania State University } \\ \text { PTB } & \text { Physikalisch-Technische } \\ & \text { Bundesanstalt, Braunschweig } \\ \text { PU } & \text { processing unit } \\ \text { PUMS } & \text { Public Use Microdata Samples } \\ \text { PUSPITEK } & \text { Training Facilities in the Centre } \\ & \text { for Research, Science, and } \\ & \text { Technology } \\ \text { PV } & \text { potential vorticity } \\ \text { PVC } & \text { polyvinyl chloride } \\ \text { PW } & \text { precipitable water } \\ \text { PWD } & \text { Public Works Department } \\ \text { PWV } & \text { precipitable water vapor }\end{array}$




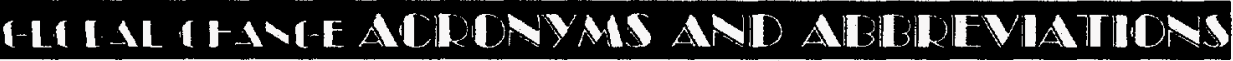

\section{Q I I}

QA

QAMS

QA/QC

QBO

QC

QLN

QN

QTL

QTO quality assurance

Quality Assurance Manageiment Staff

quality assurance/quality control quasi-biennial oscillation quality control

Quantitative Links Networks quinoline

Quantitative Trait Loci

quasi-triennial oscillation 


\begin{tabular}{|c|c|}
\hline$r^{2}$ & coefficient of determination \\
\hline R\&D & research and development \\
\hline R\&SSD & $\begin{array}{l}\text { Radiation and Safety Surveys } \\
\text { Department (ORNL) }\end{array}$ \\
\hline RA & Radar altimeter \\
\hline $\mathbf{R A}$ & Regional Association (WMO) \\
\hline RA-2 & POEM altimeter (ESA) \\
\hline RA I & $\begin{array}{l}\text { Regional Association I - Africa } \\
\text { (WMO) }\end{array}$ \\
\hline RA II & $\begin{array}{l}\text { Regional Association II - Asia } \\
\text { (WMO) }\end{array}$ \\
\hline RA IV & $\begin{array}{l}\text { Regional Association IV - North } \\
\text { and Central America (WMO) }\end{array}$ \\
\hline RAD & Radioecology Programme (NKS) \\
\hline radar & radio detection and ranging \\
\hline Radarsat & Radar Satellite (Canada) \\
\hline RADCAL & Radiation Calibration Laboratory \\
\hline RADPATH & $\begin{array}{l}\text { Biochemical Pathways of Artificial } \\
\text { Radionuclides (CEC) }\end{array}$ \\
\hline $\begin{array}{l}\text { RAE } \\
\text { RAM }\end{array}$ & $\begin{array}{l}\text { Royal Aircraft Establishment } \\
\text { random-access memory }\end{array}$ \\
\hline RAMP & $\begin{array}{l}\text { Remote Atmospheric } \\
\text { Measurements Program }\end{array}$ \\
\hline RAMP & $\begin{array}{l}\text { Remote Atmospheric Monitoring } \\
\text { Project }\end{array}$ \\
\hline RAMS & $\begin{array}{l}\text { regional atmospheric modeling } \\
\text { system }\end{array}$ \\
\hline RAMS & $\begin{array}{l}\text { Remote Atmospheric } \\
\text { Measurements Systems }\end{array}$ \\
\hline RAMS & $\begin{array}{l}\text { Results Analysis and Management } \\
\text { System (EPA) }\end{array}$ \\
\hline RANN & $\begin{array}{l}\text { Research Applied to National } \\
\text { Needs (NSF) }\end{array}$ \\
\hline RAOB & $\begin{array}{l}\text { Radiosonde or Rawindsonde } \\
\text { Observation }\end{array}$ \\
\hline RAP & Remedial Action Program (DOE) \\
\hline RAPD & $\begin{array}{l}\text { Random Amplified Polymorphic } \\
\text { DNA Technique }\end{array}$ \\
\hline RASC & Radio Atmospheric Study Centre \\
\hline $\begin{array}{l}\text { RAWG/ } \\
\text { Hydrology }\end{array}$ & $\begin{array}{l}\text { Regional Associations' Working } \\
\text { Groups on Hydrology (WMO) }\end{array}$ \\
\hline RBV & return-beam Vidicon \\
\hline $\operatorname{rcf}$ & relative centrifugal force \\
\hline $\mathbf{R C M}$ & radiative-convective model \\
\hline
\end{tabular}

\begin{tabular}{|c|c|}
\hline RCRA & Resource Conservation and \\
\hline RCRE & $\begin{array}{l}\text { Recovery Act (1976) } \\
\text { required collaborative research } \\
\text { experience }\end{array}$ \\
\hline RCS & radar cross section \\
\hline RDAAC & $\begin{array}{l}\text { Remote Data, Analysis, and } \\
\text { Archive Center }\end{array}$ \\
\hline RDBMS & $\begin{array}{l}\text { Relational Data Base Management } \\
\text { System (Oracle) }\end{array}$ \\
\hline RDP & Radar Development Project \\
\hline RDT & remote data transmission \\
\hline REFLEX & $\begin{array}{l}\text { Remote Fluvial Experimental } \\
\text { Series }\end{array}$ \\
\hline REKLIP & $\begin{array}{l}\text { Regional Climate Project (REgio- } \\
\text { KLima-Projekt) }\end{array}$ \\
\hline RES & Regional Evaporation Study \\
\hline RESA & $\begin{array}{l}\text { Scientific Research Society of } \\
\text { America }\end{array}$ \\
\hline RESSAC & $\begin{array}{l}\text { The Consequence of a Major } \\
\text { Nuclear Accident on the } \\
\text { Surrounding Environment: Soil and } \\
\text { Surface Rehabilitation (CEC) }\end{array}$ \\
\hline RET & renewable energy technologies \\
\hline RF & radio frequency \\
\hline RFE & Regional Finite Element (model) \\
\hline $\begin{array}{l}\text { RFD } \\
\text { RFLP }\end{array}$ & $\begin{array}{l}\text { Royal Forestry Department } \\
\text { restriction fragment length } \\
\text { polymorphism }\end{array}$ \\
\hline RGA & reduction gas analyzer \\
\hline RGA & relative growth rate \\
\hline RGB & red-green-blue (signal) \\
\hline RGCT & Reflective Gross Cloud Test \\
\hline RGI & regional-global interactions \\
\hline RGIS & $\begin{array}{l}\text { regional geographic information } \\
\text { system }\end{array}$ \\
\hline RGR & relative growth rate \\
\hline RH & relative humidity \\
\hline RHIESSys & $\begin{array}{l}\text { Regional Hydro-Ecological } \\
\text { Simulation System }\end{array}$ \\
\hline RI & radar interferometry \\
\hline RIC & Regional Information Center \\
\hline RICE & $\begin{array}{l}\text { Rice Cultivation and Trace Gases } \\
\text { Exchange (India) (IGAC) }\end{array}$ \\
\hline RIDGE & $\begin{array}{l}\text { Ridge Inter-Disciplinary Global } \\
\text { Experiment }\end{array}$ \\
\hline
\end{tabular}


GLIL IIATE ACLONYMS AND ABBREVIATIONS

\begin{tabular}{|c|c|c|c|}
\hline \multirow[t]{2}{*}{ RIHMI-WDC } & $\begin{array}{l}\text { All-Russian Research Institute of } \\
\text { Hydrometeorological Information- }\end{array}$ & RPN & $\begin{array}{l}\text { Division de la recherche en } \\
\text { prevision numerique }\end{array}$ \\
\hline & World Data Center & RPSS & relative partial sum of squares \\
\hline \multirow[t]{2}{*}{ RILWAS } & $\begin{array}{l}\text { Regional Integrated Lake- } \\
\text { Watershed Acidification Study }\end{array}$ & RRC & $\begin{array}{l}\text { Regional Research Centre } \\
\text { (START) }\end{array}$ \\
\hline & (EPRI) & RRCT & Reflectance Ratio Cloud Test \\
\hline RIMI & $\begin{array}{l}\text { Riso Integrated Environmental } \\
\text { Project }\end{array}$ & RRN & $\begin{array}{l}\text { Regional Research Networks } \\
\text { (START) }\end{array}$ \\
\hline \multirow[t]{3}{*}{ RIS } & reflector in space (laser beam & RRN ANT & RRN Antarctic \\
\hline & adsorption, measures $\mathrm{O}_{3}, \mathrm{CFCs}$, & RRN ART & RRN Arctic \\
\hline & etc.) & RRN CAA & RRN Central Arid Asia \\
\hline RIS & retroreflector in space & RRN CAR & RRN Caribbean \\
\hline RISC & reduced instruction set chip & RRN ESA & RRN Equatorial South America \\
\hline RISC & reduced instruction set computer & RRN MED & RRN Mediterranean \\
\hline RITS & radiatively important trace species & RRN NAF & RRN Northern Africa \\
\hline \multirow[t]{2}{*}{ RIVM } & Netherlands National Institute of & RRN OCE & RRN Oceania \\
\hline & Public Health and Environmental & RRN SAF & RRN Southern and Eastern Africa \\
\hline RK & $\begin{array}{l}\text { Protection } \\
\text { river kilometer }\end{array}$ & RRN TAM & $\begin{array}{l}\text { RRN Tropical Asian Monsoon } \\
\text { Region }\end{array}$ \\
\hline RLU & relative light unit & RRN TEA & RRN Temperate East Asia \\
\hline RM & reference material & RRN TNH & RRN Temperate Northern \\
\hline RM & river mile & & Hemisphere \\
\hline RMC & $\begin{array}{l}\text { Regional Meteorological Centre } \\
\text { (WWW) }\end{array}$ & $\begin{array}{l}\text { RRN TSA } \\
\text { RRS }\end{array}$ & $\begin{array}{l}\text { RRN Temperate South America } \\
\text { regional research sites (START) }\end{array}$ \\
\hline RMES & $\begin{array}{l}\text { Relay Mirror Experimental } \\
\text { Satellite }\end{array}$ & $\begin{array}{l}\text { RRTM } \\
\text { RS }\end{array}$ & $\begin{array}{l}\text { rapid radiative transfer model } \\
\text { remote sensing }\end{array}$ \\
\hline $\mathrm{rms}$ & $\begin{array}{l}\text { root mean square (preferred over } \\
\text { "RMS") }\end{array}$ & RSAC & $\begin{array}{l}\text { Remote Sensing Applications } \\
\text { Center (China) }\end{array}$ \\
\hline RMS & $\begin{array}{l}\text { root mean square ("rms" is } \\
\text { preferred) }\end{array}$ & $\begin{array}{l}\text { RSD } \\
\text { RSF }\end{array}$ & $\begin{array}{l}\text { residual standard deviation } \\
\text { LANL computer code }\end{array}$ \\
\hline rmse & $\begin{array}{l}\text { root mean square error (preferred } \\
\text { over "RMSE") }\end{array}$ & RSMC & $\begin{array}{l}\text { Regional/Specialized } \\
\text { Meteorological Centre }\end{array}$ \\
\hline RMSE & $\begin{array}{l}\text { root mean square error ("rmse" is } \\
\text { preferred) }\end{array}$ & $\begin{array}{l}\text { RSR } \\
\text { rss }\end{array}$ & $\begin{array}{l}\text { rotating shadowband radiometer } \\
\text { root-sum-square }\end{array}$ \\
\hline RMTC & Regional Meteorological Training & RSS & rotating shadowband spectrometer \\
\hline $\mathbf{R n}$ & $\begin{array}{l}\text { Centre } \\
\text { radon }\end{array}$ & RSSD & $\begin{array}{l}\text { Responses of Savannas to Stress } \\
\text { and Disturbance (IUBS and MAB) }\end{array}$ \\
\hline RNA & ribonucleic acid & RT & radiative transfer \\
\hline \multirow[t]{2}{*}{ RNMI } & Royal Netherlands Meteorological & RTD & real-time data \\
\hline & Institute & RTH & Regional Telecommunication Hub \\
\hline ROM & read-only memory & RTNEPH & real-time nephanalysis \\
\hline \multirow[t]{3}{*}{ ROPME } & Regional Organization for the & RUC & Roskilde University Center \\
\hline & Protection of the Marine & RUT & reflectance uniformity test \\
\hline & $\begin{array}{l}\text { Environment (IOC) } \\
\text { remotely piloted aircraft }\end{array}$ & RVAU & $\begin{array}{l}\text { Royal Veterinary and Agricultural } \\
\text { University }\end{array}$ \\
\hline RPE & Office of OECD, European & RVB & return-beam Vidicon \\
\hline & Community, and Atlantic & RWC & Regional Warning Centre \\
\hline RPM & $\begin{array}{l}\text { Office of NATO and Atlantic } \\
\text { Political-Military Affairs }\end{array}$ & RWVL & Raman Water Vapor Lidar \\
\hline rpm & revolutions per minute & & \\
\hline
\end{tabular}




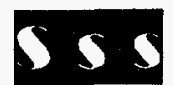

8

S\&R

S\&T

s-GCOS

SA

SABLE

SAC

SAC

SAC

SADC

SADCC

SAF

SAF

SAFCOM

SAFIRE

SAFISY

SAGE

SAHC

SAIC

SAIL

SAM

SAMBO

SAMS

SAO

SAP

SAR

SAR second (do NOT use "sec" for

"second;" use "sec" for "secant")

search and rescue

science and technology

Space-Based Global Change

Observation System

Selective Availability (on GPS

transmissions)

simulator atmospheric boundary

layer environment

Satellite Application Centre

Scientific Advisory Committee

Scientific Advisory Council (IGBP)

Southern Africa Development

Community

Southern African Development

Coordination Conference

Society of American Foresters

Southern, Central, and Eastern

Africa (START)

Southern, Central, and Eastern

Africa Regional Committee

(START)

spectroscopy of the atmosphere

using far infrared emission

Space Agency Forum on

International Space Year (1992)

Stratospheric Aerosol and Gas

Experiment

South Asia Hydrological Cycle

Science Applications International

Corporation

Steel Authority of India Limited

Stratospheric Aerosol

Measurement

Simultaneous Auroral

Multi-Balloons Observations

stratospheric and mesospheric

sounder

Smithsonian Astrophysical

Observatory (U.S.)

Scientific Advisory Panel (SARCS)

Subcommittee on Atmospheric

Research

synthetic aperture radar
SARB

SARCS

SARCS-SAP

SARSAT

SAS

SAS

SASANEE

SASS

SASI

SAT

SATOBS

SATOR

SAVE

SAW

SBC

SBC

SBIR

SBUV

SBUV-2

SC

$\mathrm{SC}$

SCA

SCANSCAT

SCAR

SCARAB

SCARABE

SCAT

SCATT

SCD

SCF surface and atmospheric radiation

budget

Southeast Asia Regional

Committee (START)

SARCS Scientific Advisory Panel

Search and Rescue Satellite

Southern Asian Region (START)

Statistical Analysis Systems

South and Southeast Asia Network for Environmental Education

Seasat A Scatterometer System

Statistical Analysis Systems

Institute (Raleigh, N.C.)

surface air temperature

Satellite Observations

Stratospheric and Tropospheric

Ozone Research

South Atlantic Ventilation

Experiment

sawlog harvest

Brazilian Society for Cartography, Geodesy, Photogrammetry, and

Remote Sensing

sum of base cations

Small Business Innovation

Research

Solar Backscatter Ultraviolet

(experiment)

Solar Backscatter Ultraviolet-2

Scientific Committee (IGBP)

standing committee

snow-covered areas

scanning scatterometer

Scientific Committee on Antarctic

Research (ICSU)

Scanner for the Radiation Budget

Scanner for Radiative Budget

Experiment

radar scatterometer

scatterometer

Scientific Computing Division

(NCAR)

Scientific Computing Facility 
TLIIL IFAYE ACDONYMS ANID AMBBDEVIATIONS

\begin{tabular}{|c|c|c|}
\hline SCIBP & $\begin{array}{l}\text { Scientific Committee for the } \\
\text { International Biological } \\
\text { Programme }\end{array}$ & $\begin{array}{l}\text { SEB } \\
\text { SEBEX }\end{array}$ \\
\hline SC-IGBP & $\begin{array}{l}\text { Special Committee of the } \\
\text { International Geosphere Biosphere }\end{array}$ & SEC \\
\hline SCM & $\begin{array}{l}\text { Programme (ICSU) } \\
\text { single-column model }\end{array}$ & sec \\
\hline SCOPE & $\begin{array}{l}\text { Scientific Committee on Problems } \\
\text { of the Environment (ICSU and } \\
\text { IGBP) }\end{array}$ & SEDAC \\
\hline SCOR & $\begin{array}{l}\text { Scientific Committee on Oceanic } \\
\text { Research (ICSU) }\end{array}$ & SEL \\
\hline SCOSTEP & $\begin{array}{l}\text { Scientific Committee on } \\
\text { Solar-Terrestrial Physics }\end{array}$ & SELPER \\
\hline SCOT & $\begin{array}{l}\text { Services Consultance en } \\
\text { Observation de la Terre }\end{array}$ & $\begin{array}{l}\text { SELV } \\
\text { SEM }\end{array}$ \\
\hline SCPP & $\begin{array}{l}\text { Seasonal to Interannual Climate } \\
\text { Prediction Program }\end{array}$ & $\begin{array}{l}\text { SEM } \\
\text { SERC }\end{array}$ \\
\hline SCRIPPS & $\begin{array}{l}\text { Scripps Institution of } \\
\text { Oceanography (University of } \\
\text { California at San Diego) }\end{array}$ & SERDP \\
\hline SCS & $\begin{array}{l}\text { Soil Conservation Service } \\
\text { (Anchorage, Alaska) (USDA and } \\
\text { DOA) }\end{array}$ & $\begin{array}{l}\text { SERI } \\
\text { SERON }\end{array}$ \\
\hline SCS & Swiss Calibration Service & SERP \\
\hline SCST & $\begin{array}{l}\text { State Commission for Science and } \\
\text { Technology (Survey of India) }\end{array}$ & SESAME \\
\hline $\begin{array}{l}\text { SD } \\
\text { SDAC }\end{array}$ & $\begin{array}{l}\text { standard deviation } \\
\text { Satellite Data Analysis Center } \\
\text { (SRB) }\end{array}$ & $\begin{array}{l}\text { SESC } \\
\text { SETAC }\end{array}$ \\
\hline $\begin{array}{l}\text { SDC } \\
\text { SDS } \\
\text { SDS } \\
\text { SDSC }\end{array}$ & $\begin{array}{l}\text { Swiss Development Corporation } \\
\text { site data system } \\
\text { sodium dodecyl sulfate } \\
\text { San Diego Supercomputer Center }\end{array}$ & $\begin{array}{l}\text { SF } \\
\text { SFC } \\
\text { SGCR }\end{array}$ \\
\hline SDSD & Satellite Data Services Division & SGM \\
\hline SDSS & Spatial Decision Support System & SGP \\
\hline SDTS & Spatial Data Transfer Standard & SGP \\
\hline $\begin{array}{l}\text { SDWA } \\
\text { SEAMEO }\end{array}$ & $\begin{array}{l}\text { Safe Drinking Water Act } \\
\text { South East Asian Ministers } \\
\text { Education Organization }\end{array}$ & $\begin{array}{l}\text { SGR } \\
\text { SH } \\
\text { SHARE }\end{array}$ \\
\hline SEAREX & Sea-Air Exchange (program) & \\
\hline Seasat & $\begin{array}{l}\text { sea satellite (an oceanographic } \\
\text { research satellite) (NASA) }\end{array}$ & SHPO \\
\hline SEASPAN & $\begin{array}{l}\text { SEAREX South Pacific Aerosol } \\
\text { Network }\end{array}$ & SHSG \\
\hline SeaStar & Spacecraft flying SeaWIFS & \\
\hline
\end{tabular}

Southeast Asian Treaty

Organization

sea-viewing wide-field sensor

State Electricity Board

Sahelian Energy Balance

Experiment

Science Executive Committee of the IWG

secant (trigonometric function) (do

NOT use "sec" for "second;" use

"s" for "second")

Socio-Economic Data and

Applications Center (CIESIN)

Standard Elektrik Lorenz (AG

Stuttgart, Germany)

Society of Latin American Remote

Sensing Specialists

small expendable launch vehicle

scanning electron microscope

space environment monitor

Smithsonian Environmental

Research Center

Strategic Environmental Research

and Development Program

Solar Energy Research Institute

Southeastern Regional Oxidant

Network

Sedimentary Rock Program

Severe Environmental Storms and

Mesoscale Experiment

Space Environment Service Center

Society of Environmental

Toxicology and Chemistry

screening factor

surface

Subcommittee on Global Change

Research

second-generation model

single Gauss point

Southern Great Plains

specific growth rate

Southern Hemisphere

Software Help for Applications,

Research, and Education

state historic preservation officer (Memphis)

Spherical Harmonic Spatial Grid (model) 


\section{TLILILAYE ACDONYMS AND AMBREVIATIDNS}

SI

SI

SI

SIAM

$\mathrm{SiB}$

SIDS

SIFE

SIG-WAIS

SIMD

SIO

$\mathrm{SiO}_{3}$

SIP

SIR

SIR

SIR

SIR-C

SIROS

SIRS

SISEX

SIT

SITE

SIV

SIZ

SJVF

SK

SLA

SLAR

SLB

SLEJF

SLIES

SLP

SLR

SLR
International System of Units

(Systeme International d'Unites)

(standard metric units; replaced

mks and mksa systems)

Smithsonian Institution

(Washington, D.C.)

solar influences

Society for Industrial and Applied Mathematics (Philadelphia)

simple biosphere (model)

Sustainable Development of Small

Island Developing States

Second ISCCP Field Experiment

Special Interest Group-Wide Area

Information Server

Single Instruction, Multiple Data

(system) (processor)

Scripps Institution of

Oceanography (San Diego, Calif.)

silicate

Solar Influences Program

Scientific Information and

Retrieval (system)

Shuttle Imaging Radar

Spaceborne Imaging Radar

Shuttle Imaging Radar-C

solar and infrared radiation

observation stations

satellite infrared spectrometer

Shuttle Imaging Spectrometer

Experiment

Servizio di Taratura in Italia

Satellite Instructional Television

Experiment

solar and interplanetary variability

Seasonal Ice Zone

Veterinary Agricultural Research

Council

Saskatchewan

Special Libraries Association

side-looking airborne radar

shallow land burial

Sri Lanka Environmental

Journalists Forum

stratospheric limb infrared

emission spectrometer

sea-level pressure

satellite laser ranging

sea level rise
SLS

SLURP

SMC

SMCRA

SME

SME

SMM

SM-MIMD

SMMR

SMMR

SMO

SMOS

SMOW

SMS

SMS/GOES

SMY

SN

SNAS

SNL

SNL/PPG

SNOTEL

SNR

SO

$\mathrm{SO}_{2}$

$\mathrm{SO}_{4}$

SOA

SOC

SOCC

SOCEX

SOHO

SOI

SOI

SOLSTICE

SOMMA

SOP stereo line scanner

Simple Linear Unit Reservoir

Program

System Management Center

Surface Mining Control and

Reclamation Act

Society of Mining Engineers

Solar Mesosphere Explorer

Solar Maximum Mission

shared memory, multiple

instruction, and multiple data

(computer)

scanning multichannel microwave

radiometer

scanning multifrequency

microwave radiometer

Samoa Observatory (American

Samoa) (CMDL)

surface meteorological observation

system

isotope standard for $\mathrm{H}_{2} \mathrm{O}$

Synchronous Meteorological

Satellite

SMS/Geostationary Operational

Environmental Satellite

solar maximum year

Space Network

Strategic National Alternatives

Study

Sandia National Laboratories

SNL/Parallel Processing Group

snow survey and remote telemetry

signal-to-noise ratio

Southern Oscillation

sulfur dioxide

sulfate radical

state-of-the-art

soil organic carbon

Satellite Operation Command and

Control

Southern Ocean Cloud Experiment

Solar Heliospheric Observatory

Southern Oscillation Index

Survey of India

Solar Stellar Irradiance

Comparison Experiment

single-operator multiparameter

metabolic analyzer

standard operating procedures 


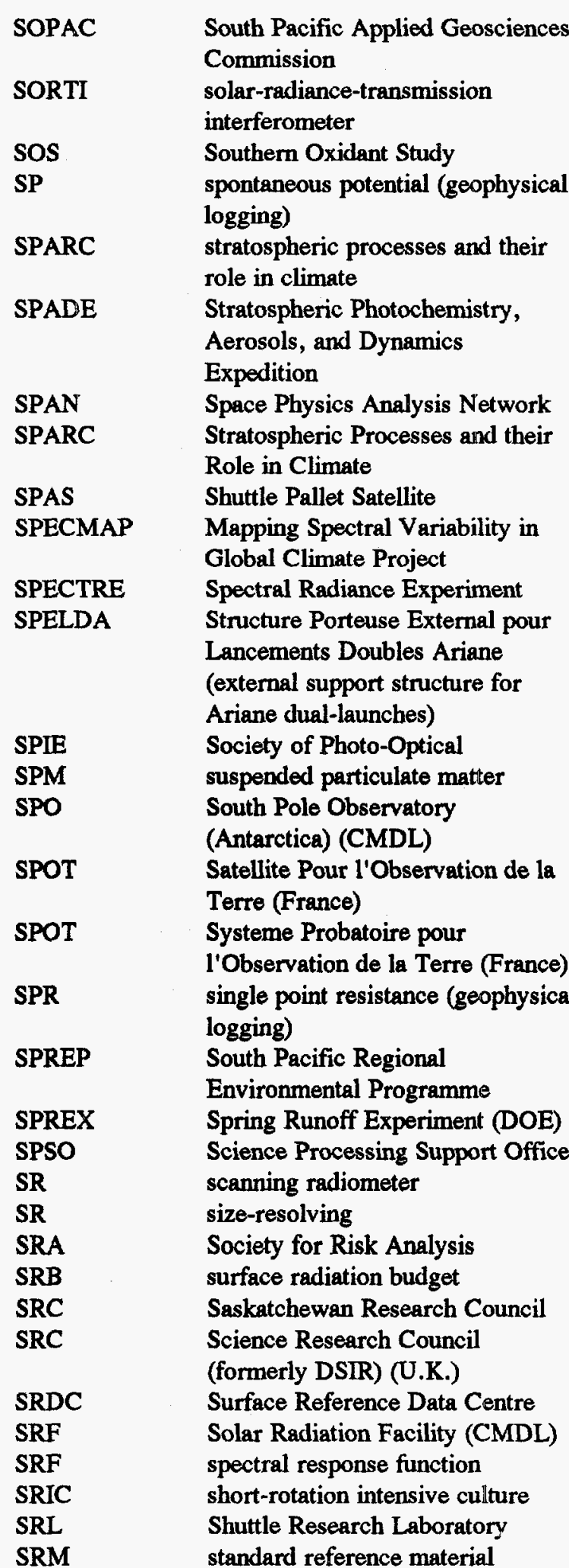

SRP

SRS

SRS1

SRWCP

SS

SSA

SSALT

SSARR

SSB

SSB

SSBUV

SSC

SSEC

SSG

SSHT

SSI

SSM

SSM/I

SSM/T

SSMR-SSM/I

SSP

SSSA

SSSD

SST

SSU

SSURGO

STA

STA

STAP Scientific and Technical Advisory

STAR Space Three-Axis Accelerometer

START System for Analysis, Research,

START-SC START Standing Committee STEM STEP Panel (GEF) for Research and Training (IGBP, WCRP, and HDP)

soluble reactive phosphorus

Statistical Reporting Service

South Research Station 1 (WOCE)

Short Rotation Woody Crop

Program

stainless steel (flasks) .

singular spectrum analysis

solid-state (radar) altimeter

Streamflow Synthesis and

Reservoir Regulation (model)

Space Science Board (NRC)

Space Studies Board

shuttle solar backscatter ultraviolet (radiometer)

(JGOFS, PAGES, and IGBP)

Space Science and Engineering

Center

Scientific Steering Group

Sea Surface Height (above some

reference ellipsoid)

Spectral Statistical Interpolation

special sensor microwave

SSM/temperature

scanning multichannel microwave radiometer-SSM/I

scanning spectral polarimeter

Soil Science Society of America

state soil survey data base

sea-surface temperature

stratospheric sounding unit

(USDA and SCS)

Science and Technology Agency

(Japan)

Strategic Transportation Analysis (integrated transportation networks solar terrestrial environment model Solar Terrestrial Energy Program 


\section{GLIL IFIVE ACRONYMS AND AMBBLEVIATIIDNS}

STIB

STIKSCAT

STIP

STORET

STORM

STORM-FEST

STP

STP

STRESS

STRI

STRIVE

STSB

SUNY

SUP

SUSIM

SVAT

SVATS

SW

SWACOM

SWADE

SWCA

SWCC

SWDA

SWE

SWEDAC

SWF

SWG

SWG
Stratosphere-Troposphere

Interaction and the Biosphere

(IGBP)

stick scatterometer

Study of Travelling Interplanetary

Phenomena

Storage and Retrieval System

(water quality data; EPA)

Storm-scale Operational and

Research Meteorology (program)

Storm-scale Observations Regional

Measurement Program-Fronts

Experiment Systems Test

standard temperature and pressure $\left(0^{\circ}\right.$ and $\left.1 \mathrm{~atm}\right)$

Subsurface Transport Program (DOE and OHER)

sediment transport events on

shelves and slopes

Smithsonian Tropical Research

Institute

Science Teachers Research

Involvement for Vital Education

(now TRAC)

Scientific and Technical Services

Branch (BoM)

State University of New York

soluble unreactive phosphorus

Solar Ultraviolet Spectral

irradiance monitor

soil vegetation atmosphere transfer (model) (BAHC)

Surface Vegetation Atmosphere

Transfer Scheme

shortwave

Standard Water Column Model

Surface Wave Dynamic

Experiment

Soil and Water Conservation Act (1977)

Second World Climate Conference (1990)

Solid Waste Disposal Act

shallow-water equations

Swedish Board for Technical

Accreditation

social welfare function

Science Working Group

Standards Working Group
SWH

SWIR

SWIRLS

SWSA significant wave height

short wavelength infrared

stratospheric wind infrared limb sounder

solid waste storage area 


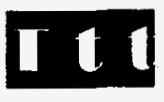

T/P SWT

T

TA

TABLE

TAC

TAE

TAGA

Talk

TAM

TAO

TAP

TAPPI

TBD

TCA

TCDC

TCLP

TCO

$\mathrm{TCO}_{2}$

TCP

TCP/IP

TCR

TDDR

TDL

TDLAS

TDMA

TDR

TDRS

TDRSS

TDS
TOPEX/Poseidon Science Working

Team

tonne

trace analytical

Tsukuba Atmospheric Boundary

Layer Experiment

Tactical Air Command (U.S. Air

Force bases) (now ACC)

Transportable Applications

Executive (a computer user interface)

trace atmospheric gas analyzer

total alkalinity

Tropical Asian Monsoon Region

(START)

Tropical Atmosphere Ocean

Transboundary Air Pollution (project)

Technical Association of the Pulp and Paper Industry

to be determined

trichloracetic acid

technical cooperation among

developing countries

Toxicity Characteristic Leaching

Procedure (EPA)

Technical Cooperation Activities

(WMO)

total $\mathrm{CO}_{2}$

Tropical Cyclone Programme

(WMO)

Transmission Control

Protocol/Internet Protocol

tonnes of coal replacement

total direct diffuse radiometer

Techniques Development

Laboratory

tunable diode laser absorption

spectrometer

tandem differential mobility

analyzer

time domain reflectometry

Tracking and Data Relay Systems

Tracking and Data Relay Satellite

System

total dissolved solids
TDY

TEA

TEA-COM

TEAM

TEAMA

TEC

TECO

TECO

TED

TED

TEDDY

TEESE

TEGD

TEHM

TEM

TERI

TERLS

TERRA

TERSE

TES

TF

TFAP

Tflops

$\mathrm{Tg}$

TG

TGCR

TGCT

TGDDIS

TGI temporary duty

Temperate East Asia (START)

Temperate East Asia-Planning

Committee (START)

Technology Base Enhancement for

Autonomous Machines

Terrestrial Ecosystems in Monsoon

Asia

total electron content

Terrestrial Ecological Research

Initiative

terrestrial ecosystems

Technological and Economic

Dynamics

total energy detector

TERI Energy Data Directory

Yearbook

TERI Energy Economy Simulation

Evaluation

Technical Enforcement Guidance

Document (EPA)

Terrestrial Ecosystem Hydrology

Model

Terrestrial Ecosystem Model

(GAIM)

Tata Energy Research Institute

Thumba Equatorial Rocket

Launching Station (India)

Terrestrial Ecosystem Regional

Research and Analysis

(Laboratory)

Tunable Etalon Remote Sounder

tropospheric emission spectrometer throughfall

Tropical Forestry Action Plan

(coordinated by FAO)

One teraflops equals 1 trillion

$\left(10^{12}\right)$ floating point operations per second

Teragrams or million tonnes

trace gas

thermal gross cloud restoral

thermal gross cloud test

Trace Gas Dynamics Data

Information System

temperature growth index 


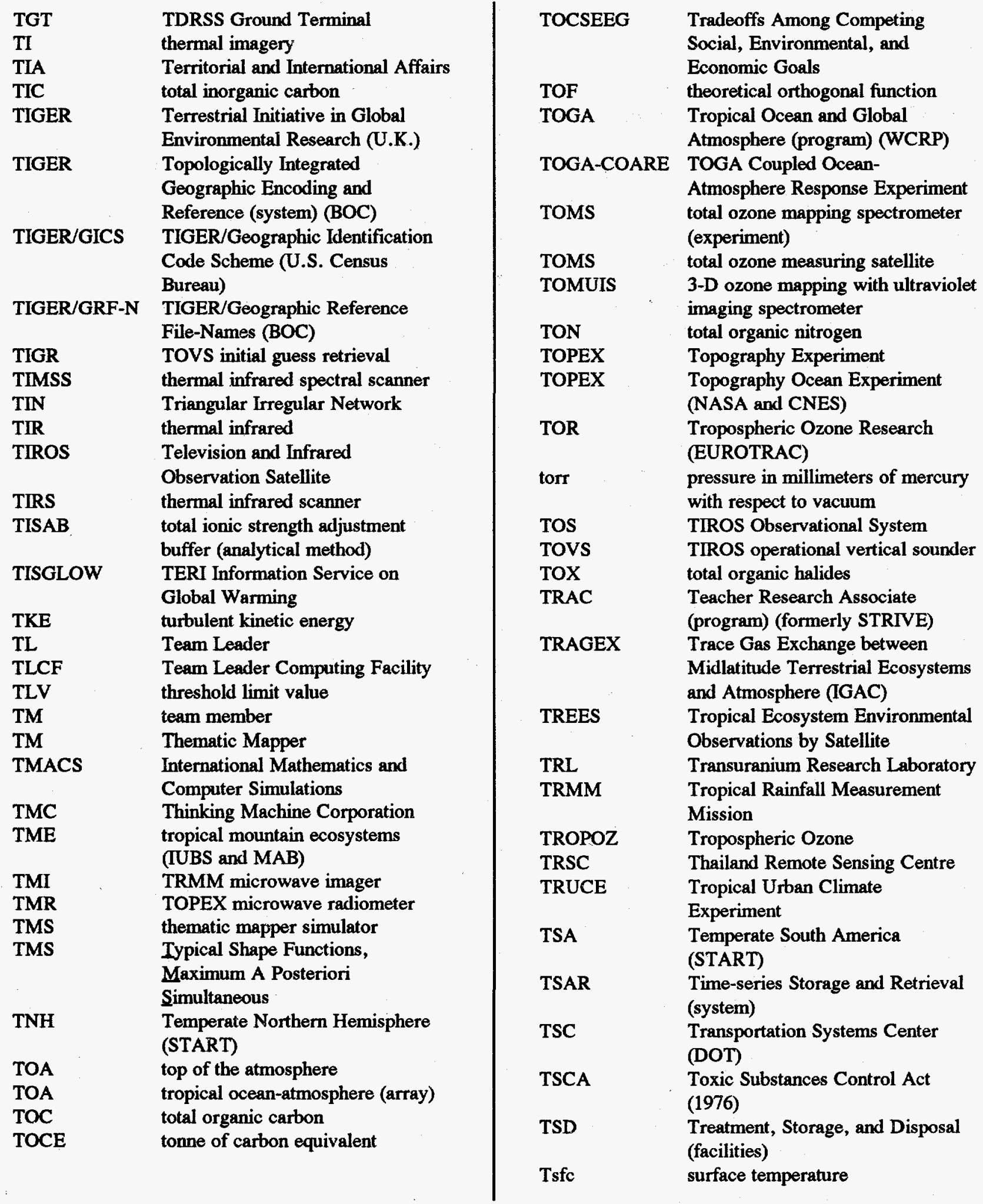


TSI

TSP

TSP

TSS

TSS

TT

TTO

TTO

TUNES

TUR

TUT

TVA

TWAS

TWP

TWPO

TWRA

TWT
Thermo Systems, Inc.

total soluble phosphorus

total suspended particles

total suspended solids

Total Surveying Systems

task team

total toxic organic (compounds)

transient tracers in the ocean

Thomas Washington's Unexpected Nautical Extravaganza South-north (expeditions)

thermal uniformity restoral

thermal uniformity test

Tennessee Valley Authority (U.S.

Independent)

Third World Academy of Science

tropical Western Pacific

Tropical Western Pacific Ocean

Tennessee Wildlife Resources

Agency

Traveling Wave Tube 


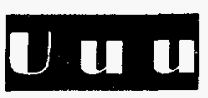

\author{
UAF \\ UAOB \\ UARP \\ UARS \\ UATI \\ UAV \\ UC
}

UCAR

UCI

UCLA

UCR

UCS

UCSB

UCSF

UDF

UFACNC

UGM

UH

UHF

UIC

UIC

UI/CSRD

UITA

U.K.

UKAEA

UKMO

ULST
University of Alaska-Fairbanks

Upper Air Observation

Upper Atmosphere Research

Program

Upper Atmosphere Research

Satellite (NASA)

Union des Associations Techniques

Internationals

unmanned aerospace vehicle

University of California

University Corporation for

Atmospheric Research

University of California, Irvine

University of California at Los

Angeles (U.S.)

University of California at

Riverside

Secretariat to the U.S. National

Commission

University of California, Santa

Barbara

University of California at San

Francisco

UAV demonstration flights

ultrafine aerosol condensation

nucleus counter

University of Gadjah Mada,

Indonesia

University of Heidelberg

(Germany)

ultra high frequency

Ukpeaguik Inupiat Corp.

Underground Injection Control (program)

University of Illinois/Center for

Supercomputing Research and

Development

Union of International Technical

Associations

United Kingdom (preferred over

"UK"; use as adjective only; spell out as noun)

U.K. Atomic Energy Authorities

U.K. Meteorological Office

uniform low stratus test
UMTA Urban Mass Transportation

Administration (DOT)

UMTRAP Uranium Mill Tailings Remedial

Action Program

UMTRCA Uranium Mill Tailings Radiation

Control Act

UN

UNARS

United Nations

UNCED United Nations Conference on

Environment and Development

UNCHS United Nations Commission for

Human Settlement

UNCTAD United Nations Commission on

Trade and Development

UNCTAD United Nations Conference on

Trade and Development

UNDP United Nations Developmental

Program

UNDRC Office of the United Nations

Disaster Relief Coordinator

UNEC

United Nations Economic

Committee

UNEP

UNESCO

UNFCCC

UNFPA

UN GA

UNGCRP

UNHCR

United Nations Environmental

Programme (ICSU)

United Nations Educational,

Scientific, and Cultural

Organization

UN Framework Convention on

Climate Change

United Nations Program for

Population

UN General Assembly

UN Global Change Research

Program

Office of the United Nations High

Commissioner for Refugees

UNICEF

UNIDO

United Nations Children's Fund

United Nations Industrial

Development Organization

UNIFEM United Nations Development Fund for Women

UNISIST United Nations System for Scientific and Technological Information 


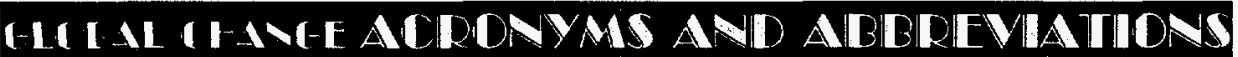

\begin{tabular}{|c|c|c|}
\hline UNITAR & $\begin{array}{l}\text { United Nations Institute for } \\
\text { Training and Research }\end{array}$ & USDOD \\
\hline UNJSPF & $\begin{array}{l}\text { United Nations Joint Staff Pension } \\
\text { Fund }\end{array}$ & USDOE \\
\hline UNM & University of New Mexico & USDOI \\
\hline UNO & United Nations Organization & \\
\hline UNP & United Nations Political Affairs & USDW \\
\hline UNRWA & $\begin{array}{l}\text { United Nations Relief and Works } \\
\text { Agency }\end{array}$ & USEPA \\
\hline UNSCEAR & $\begin{array}{l}\text { United Nations Scientific } \\
\text { Committee on the Effects of } \\
\text { Atomic Radiation }\end{array}$ & $\begin{array}{l}\text { USFS } \\
\text { USFWS } \\
\text { USGCRP }\end{array}$ \\
\hline UNSO & $\begin{array}{l}\text { United Nations Sudano-Sahelian } \\
\text { Office }\end{array}$ & \\
\hline UNTAB & $\begin{array}{l}\text { United Nations Technical } \\
\text { Assistance Board }\end{array}$ & $\begin{array}{l}\text { USIA } \\
\text { US-IGBP }\end{array}$ \\
\hline $\begin{array}{l}\text { UNU } \\
\text { UPS } \\
\text { UPU }\end{array}$ & $\begin{array}{l}\text { United Nations University } \\
\text { uninterruptible power supply } \\
\text { Universal Postal Union }\end{array}$ & USJGOFS \\
\hline URAS & (a commercial $\mathrm{CO}_{2}$ analyzer) & USMAB \\
\hline $\begin{array}{l}\text { URI } \\
\text { URL }\end{array}$ & $\begin{array}{l}\text { University of Rhode Island } \\
\text { Uniform Resource Locator } \\
\text { (Mosaic) }\end{array}$ & $\begin{array}{l}\text { USMAPS } \\
\text { USN }\end{array}$ \\
\hline URSI & $\begin{array}{l}\text { International Union of Radio } \\
\text { Science }\end{array}$ & $\begin{array}{l}\text { USNC/IHP } \\
\text { USNC/SH }\end{array}$ \\
\hline US-AID & $\begin{array}{l}\text { U.S. Agency for International } \\
\text { Development }\end{array}$ & USNMAS \\
\hline $\begin{array}{l}\text { U.S. } \\
\text { USACE }\end{array}$ & $\begin{array}{l}\text { United States } \\
\text { U.S. Army Corps of Engineers } \\
\text { (DOD) }\end{array}$ & $\begin{array}{l}\text { USO } \\
\text { U.S.S.R. }\end{array}$ \\
\hline USAF & U.S. Air Force & \\
\hline USBCF & $\begin{array}{l}\text { U.S. Bureau of Commercial } \\
\text { Fisheries }\end{array}$ & $\begin{array}{l}\text { USWB } \\
\text { UT }\end{array}$ \\
\hline USBM & U.S. Bureau of Mines & UT \\
\hline USBR & $\begin{array}{l}\text { U.S. Bureau of Reclamation } \\
\text { (USDD) }\end{array}$ & $\begin{array}{l}\text { UTC } \\
\text { UTM }\end{array}$ \\
\hline $\begin{array}{l}\text { USCG } \\
\text { USCGS }\end{array}$ & $\begin{array}{l}\text { U.S. Coast Guard } \\
\text { U.S. Coast and Geodetic Survey }\end{array}$ & UTM \\
\hline USDA & U.S. Department of Agriculture & uv \\
\hline USDA-SCS & $\begin{array}{l}\text { U.S. Department of Agriculture- } \\
\text { Soil Conservation Service }\end{array}$ & $\begin{array}{l}\text { UV } \\
\text { uv-A }\end{array}$ \\
\hline USDC & $\begin{array}{l}\text { U.S. Department of Commerce } \\
\text { (also DOC and USDOC) }\end{array}$ & $\begin{array}{l}\text { uv-B } \\
\text { UVR }\end{array}$ \\
\hline USDD & $\begin{array}{l}\text { U.S. Department of Defense (also } \\
\text { USDOD and DOD) }\end{array}$ & $\begin{array}{l}\text { uv-vis } \\
\text { UW }\end{array}$ \\
\hline USDI & $\begin{array}{l}\text { U.S. Department of the Interior } \\
\text { (also DOI and USDOI) }\end{array}$ & $\begin{array}{l}\text { UWG } \\
\text { UW/UD }\end{array}$ \\
\hline USDOC & $\begin{array}{l}\text { U.S. Department of Commerce } \\
\text { (also DOC and USDC) }\end{array}$ & \\
\hline
\end{tabular}

U.S. Department of Defense (also DOD and USDD)

U.S. Department of Energy (also DOE)

U.S. Department of the Interior (also DOI and USDI) usable supply of drinking water U.S. Environmental Protection Agency (also EPA) U.S. Forest Service (USDA) U.S. Fish and Wildlife Service U.S. Global Change Research Program

U.S. Geological Survey (DOI)

U.S. Information Agency

U.S. Geosphere/Biosphere Program

U.S. Joint Global Ocean Flux Study

U.S. Man and the Biosphere

U.S. Map Analysis Package

System

U.S. Navy

U.S. National Committee for IHP

U.S. National Committee on Scientific Hydrology

U.S. National Map Accuracy

Standard

ultra-stable oscillator

Union of Soviet Socialist Republics (now Russian Federation)

U.S. Weather Bureau universal time

University of Tennessee

Universal Time Coordinate

Unified Transport Model

university transverse mercator (cartography)

ultraviolet (preferred over "UV")

ultraviolet ("uv" is preferred)

ultraviolet type A

ultraviolet type B

ultraviolet radiation

ultraviolet-visible (spectroscopy)

University of Wisconsin

user working group

University of Wisconsin/University of Denver 


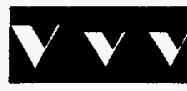

VAD

VAM

VAMP

VAS

VEI

VCP

VCP (ES)

$\mathrm{VCP}(\mathrm{F})$

vfr

vhf

VHRR

VHSA

VIRS

VIRSR

VIS

VISSR

VLBI

VLSI

VNIR

VOC

VOS

VSSC

VTIR

VTPR

VTR velocity azimuth display

vesicular-arbuscular mycorrhiza

Validation of Model Project

(IAEA)

VISSR atmospheric sounder (GOES)

volcano explosivity index

Voluntary Cooperation Program

(Equipment and Services) (UN)

Voluntary Cooperation Program

(Equipment and Services) (UN)

Voluntary Cooperation Program (fund)

very far radiation

very high frequency

very high resolution radiometer

variable hydrologic source areas

visible infrared scanner

visible infrared scanning

radiometer

visible

visible and infrared spin scan radiometer (GOES)

very long baseline interferometry very large scale integration visible and near infrared volatile organic compound voluntary observing ship Vikram Sarabhai Space Center visible and thermal infrared radiometer

vertical temperature profile radiometer

video tape recording 


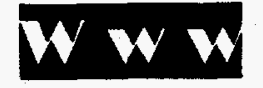

w watt

WAIS West Antarctic Ice Sheet (project) (PAGES)

WAMEX West African Monsoon Experiment

WARC

WATOX

WATFLOOD

WATSTORE

WB

WBC

WBDCS

WBIS

WBW

WCAP

WCASP

WCDMP

WCDP

WCIP

WCIRP

WCP

WCRP

WCRP-JSC Joint Scientific Committee

WDC

WDSD

WEAVE Water and Energy: Atmospheric,

WEBB

WECC (WCRP)

World Data Center (ICSU)

Water Data Sources Directory (USGS) Vegetative, and Earth Interactions

World Administrative Radio

Conference

Western Atlantic Ocean

Forecasting System (Canada)

Water Data Storage and Retrieval

System (USGS)

World Bank

Wide-Band Data Collection System

Walker Branch Information System

Walker Branch Watershed

(introduced as an "experimental

facility")

World Climate Applications

Program (WMO)

Monitoring Program (WMO)

World Climate Data Program

World Climate Impact Program

World Climate Impact Assessment

and Response Strategies Program

(WMO)

World Climate Research

Programme (WMO, ICSU, and

IOC)

Water, Energy, and

Biogeochemical Budget (USGS)

Western European Calibration

Cooperation
WEFAX

WENPEX

WEO

WES

WESTPAC

WET

WETNET

WEV

WEVS

WFC

WFEO

WFF

WFOV

WFP

WG

WGEP

WGDM

WGGC

WGNE

WGRF

WHO

WHOI

WHP

WIHG

WILASS

WIND

WINDII

WIPO

WIRP

WISP weather facsimile

Western North Pacific Cloud-

Radiation Experiment

Western European Organization

(ECOSOC region comprising

W. Europe, Canada, U.S., and

Japan)

Waterways Experiment Station

(USACE)

IOC - Programme Group on the

Western Pacific

waste extraction test

Network for Distribution and Joint

Analysis of SSM/I Data (MSFC)

Water Energy-Vegetation

Wetland Ecosystem Vulnerability

Study

World Food Council (UN)

World Federation of Engineering

Organizations

Wallops Flight Facility

Wide Field of View

World Food Program

working group

working group on energy policy

working group on data

management

working group on global change

working group on numerical

experimentation

working group on radiative fluxes

World Health Organization

Woods Hole Oceanographic

Institution

WOCE Hydrographic Programme

Waclia Institute of Himalayan

Geology

Water Infiltration in Layered

Subfreezing Snow (program)

weather information display

Wind Imaging Interferometer

World Intellectual Property

Organization

Watershed Integrated Research

Program (DOE)

Winter Icing and Storms Programs 


\section{TLI IL IFIE ACRONYMS AND ABBDEVIATIONS}

\begin{tabular}{|c|c|}
\hline WITs & $\begin{array}{l}\text { World Ionosphere Thermosphere } \\
\text { Study }\end{array}$ \\
\hline WMC & $\begin{array}{l}\text { World Meteorological Center } \\
\text { (WWW) }\end{array}$ \\
\hline WMO & $\begin{array}{l}\text { World Meteorological } \\
\text { Organization (ICSU) }\end{array}$ \\
\hline WMS & World Magnetic Survey \\
\hline WOCE & $\begin{array}{l}\text { World Ocean Circulation } \\
\text { Experiment (WCRP) }\end{array}$ \\
\hline WOCE/HP & WOCE/Hydrographic Program \\
\hline WODC & World Ozone Data Center \\
\hline wovo & $\begin{array}{l}\text { World Organization of Volcano } \\
\text { Observatories }\end{array}$ \\
\hline WP & wind profilers \\
\hline WPCF & Water Pollution Control Federation \\
\hline WPL & $\begin{array}{l}\text { Wave Propagation Laboratory } \\
\text { (NOAA) }\end{array}$ \\
\hline WPR & Water Resource Planning \\
\hline WRC & Water Resources Council \\
\hline WRC & World Radiation Center \\
\hline WRD & $\begin{array}{l}\text { Water Resources Division (USGS } \\
\text { and DOI) }\end{array}$ \\
\hline WRDD & $\begin{array}{l}\text { Water Resources Development } \\
\text { Department (MAW) }\end{array}$ \\
\hline WRI & $\begin{array}{l}\text { World Resources Institute } \\
\text { (Washington, D.C.) }\end{array}$ \\
\hline WRR & World Radiometric Reference \\
\hline WRRC & Water Resources Research Center \\
\hline WSC & Water Survey of Canada \\
\hline WSI & whole-sky imager \\
\hline WSMC & Western Space and Missile Center \\
\hline WST & Whitecap simulation tank \\
\hline WTH & whole-tree harvest \\
\hline WTR & Western Test Range (NASA) \\
\hline WUA & weighted usable area \\
\hline WVR & water vapor radiometer \\
\hline WWB & Women's World Banking \\
\hline WWF & World Wildlife Fund \\
\hline ww & World Weather Watch (WMO) \\
\hline WV & World Wide Web \\
\hline
\end{tabular}


GL I IL I HATE ACrONYMS AND ABBREVIATIONS

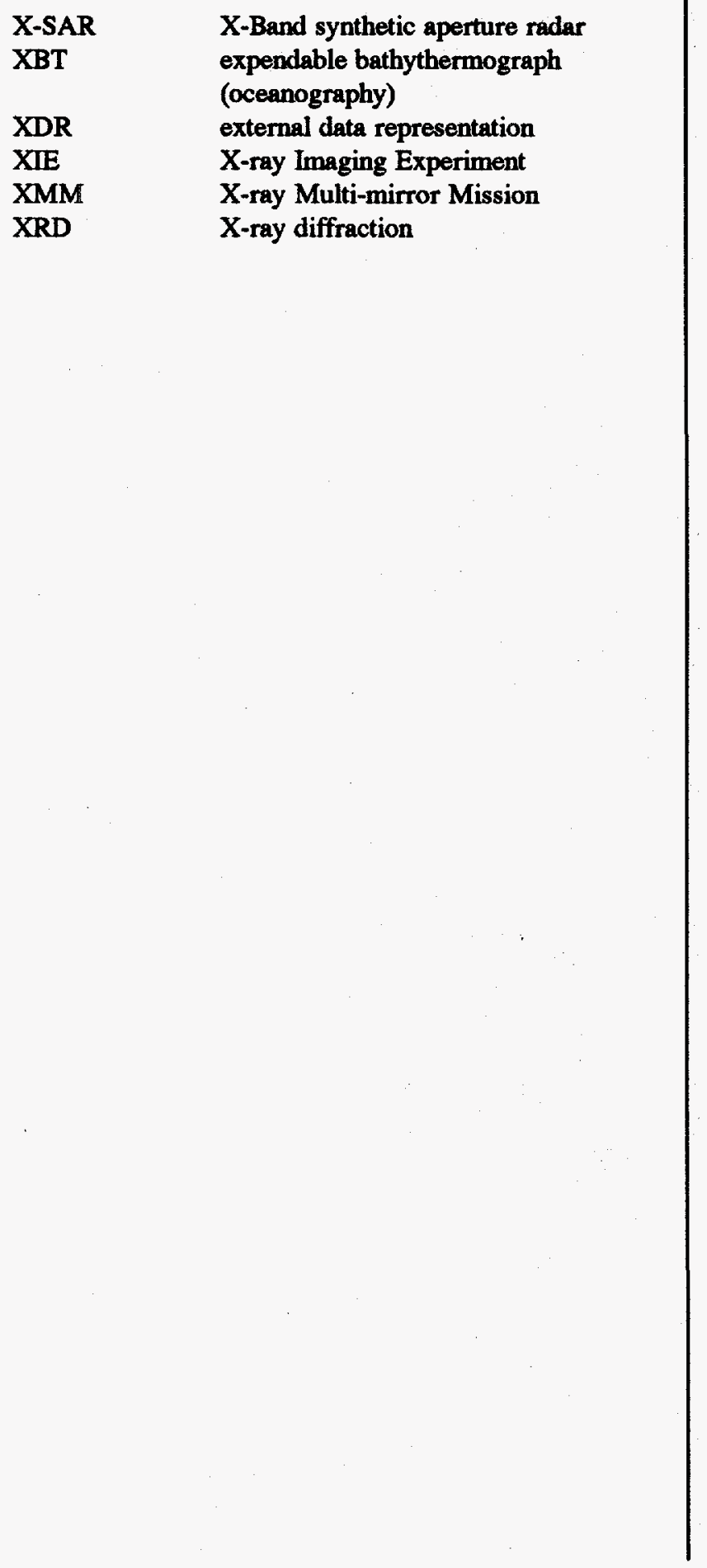

68

Carbon Dioxide Information Analysis Center (CDIAC) 


\section{(-II IL G-IVE ACDONYMS ANI) ABBDEVIATIONS}

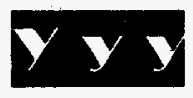

year

(do not abbreviate this word)

yr

year (spell out) 


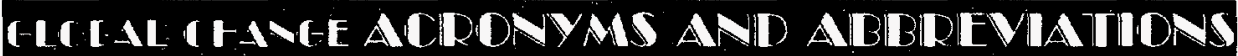

ZZX

ZEEI

Exclusive Economic Zone of Indonesia

ZPC

zero point of change (hydrous iron and aluminum oxides) 
APPENDIX A. CARBON DIOXIDE INFORMATION ANALYSIS CENTER 
The Carbon Dioxide Information Analysis Center (CDIAC), located in the Environmental Sciences Division of Oak Ridge National Laboratory (ORNL), has been in operation since 1982. CDIAC provides the international research, policy, and education communities with information about the evaluation of environmental issues associated with elevated atmospheric carbon dioxide $\left(\mathrm{CO}_{2}\right)$ and other greenhouse gases, including potential climate change. The U.S. Department of Energy's (DOE) Environmental Sciences Division funds CDIAC to support its Global Change Research Program. CDIAC is one of three global change information programs in ORNL's Environmental Information Analysis Program. The other two programs are the ORNL Earth Observing System Distributed Active Archive Center and the Atmospheric Radiation Measurement (ARM) Archive.

CDIAC's principal information products are quality-assured, fully documented numeric data packages (NDPs) and computer model packages (CMPs). These products provide comprehensive documentation of benchmark data sets and models provided by investigators from around the world. NDPs consist of written documentation and transfer media containing machine-readable text, retrieval codes, and data files. CDIAC has produced more than 50 of these specialized NDPs and has supplied copies of NDPs and CMPs to thousands of researchers, policymakers, students, and educators worldwide.

A NDP contains sections with the following information:

- Contributors

- Keywords

- Background information

- Source and scope of data

- Applications of data

- Data limitations and restrictions
- References

- Literature reprints

- Summary of data checks

- Ordering information

- Transfer media descriptions
- Data retrieval programs

- Verification of data transport

- Tabular listings and graphic output

The most critical components of the NDP process are quality assurance, documentation of the data, and thorough review and approval by the principal investigators before distribution of their repackaged data sets. CDIAC puts the data through a rigorous and extensive quality assurance review. After the data are checked, they are documented with text, data listings, illustrations, and computer codes. Unabridged reprints of pertinent literature are included with the documentation. Individuals contributing data participate in the documentation process, and no NDP is distributed without written permission from the data contributor. NDPs are updated as new data become available.

In addition to evaluating data and producing digital NDPs and CMPs, CDIAC's activities include distributing $\mathrm{CO}_{2}$-related reports and producing the newsletter CDIAC Communications, which is distributed to more than 10,000 subscribers in approximately 150 countries. CDIAC has also published Trends '93: A Compendium of Data on Global Change, Catalog of Data Bases and Reports, Catalog of Numeric Data Packages and Computer Model Packages, Glossary: Carbon 
Dioxide and Climate, and DOE Research Summary (a four-page newsletter highlighting a specific DOE-sponsored research project). CDIAC also produces ARM Outreach, a newsletter for DOE's Atmospheric Radiation Measurement Program.

Since 1985, CDIAC staff have responded to tens of thousands of requests for information. CDIAC staff are available to discuss both general and technical aspects of issues related to the carbon cycle; $\mathrm{CO}_{2}$, methane, and other trace gas emissions; and other climate change topics, including data and information management issues.

The goal of DOE's Global Change Research Program (GCRP) is to develop sound scientific information for policy formation and government action in response to changes in atmospheric $\mathrm{CO}_{2}$. GCRP's mission during the past decade has been to (1) elucidate processes that control the global carbon cycle and provide predictions of future atmospheric $\mathrm{CO}_{2}$ change; (2) develop data and models of the processes by which changes in the Earth's radiative balance may affect climate at global and regional scales and predict rates of potential climate change; and (3) develop data and models required to define and predict the combined effect of climate and $\mathrm{CO}_{2}$ on plants, crops, and ecosystems.

CDIAC operates World Data Center-A for Atmospheric Trace Gases. The International Council of Scientific Unions established the World Data Centers (WDCs) in 1956. The U.S. National Academy of Sciences oversees World Data Center-A, which includes 12 data centers throughout the United States. As a WDC, CDIAC can better obtain and disseminate information.

CDIAC shares responsibility with the ORNL Central Research Library for operating a Regional Information Centre established by the International Geosphere-Biosphere Programme (IGBP). There are 50 IGBP information centers in 37 countries, which expand the information services for the international global change community. 

APPENDIX B. CDIAC ORDER FORM 


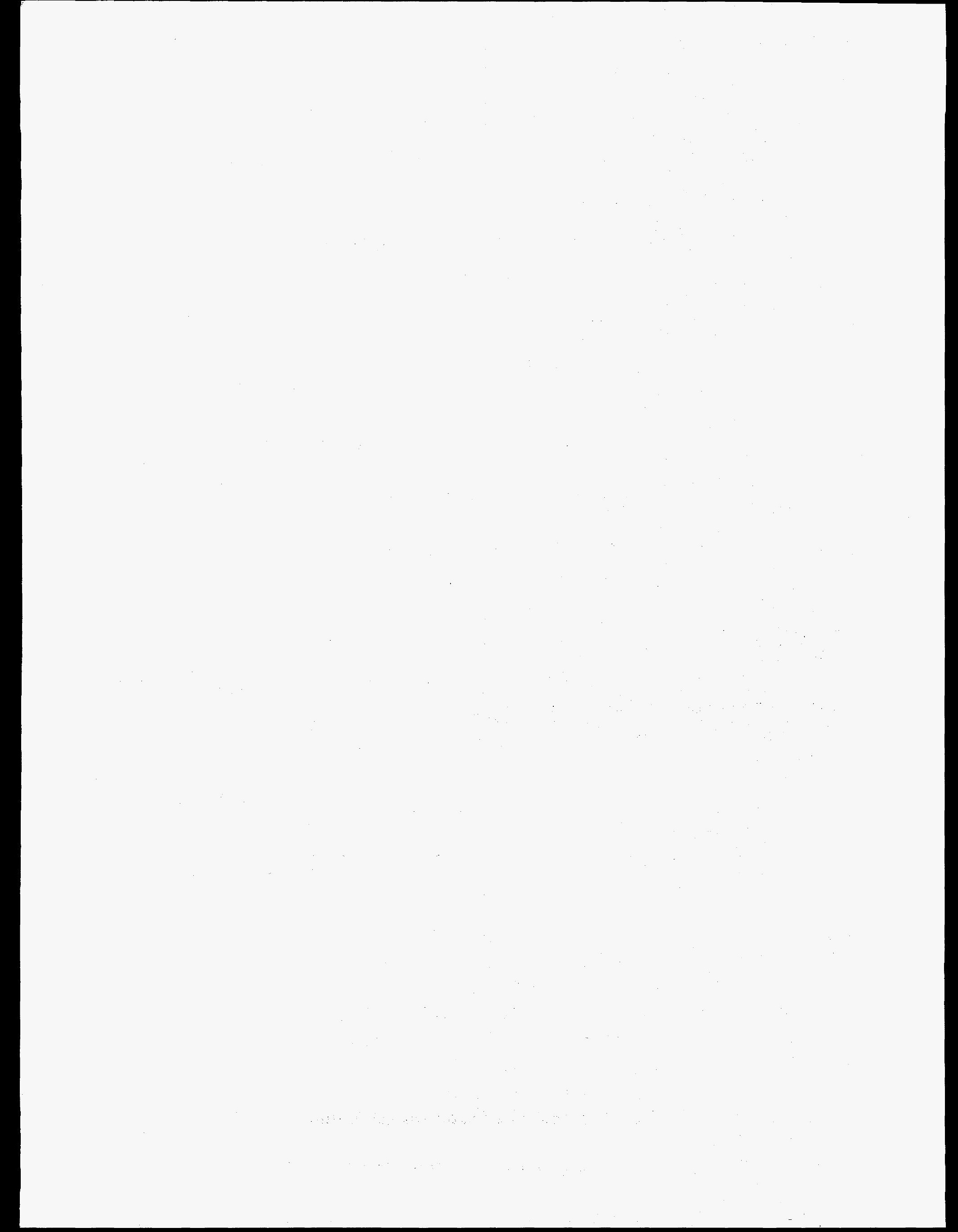




\section{CDIAC Order Form}

Check boxes for materials you would like to receive. All information is provided free of charge.

ARM Outreach, newsletter of the ARM (Atmospheric Radiation Measurement) Program

C Catalog of Data Bases and Reports

CDIAC Catalog of Numeric Data Packages and Computer Model Packages

CDIAC Communications (newsletter)

DOE Research Summary (newsletter)

$\square \quad$ Fact Sheet: Specialty Newsletters - Global Studies and Climate Change

Glossary: Carbon Dioxide and Climate

Selected Translated Abstracts of Russian-Language Climate-Change Publications: Vol 1. Surface Energy Budget, Vol. 2 Clouds

Trends '93: A Compendium of Data on Global Change

Trends '93 data on DOS-formatted floppy diskette: $\square$ 31/2" $\square 51 / 4$ "

$\square$ Instructions for accessing CDIAC's File Transfer Protocol (FTP) Area on the Internet

Name

(Last) (First)

(M.I.)

Organization

Mailing Address

City

State

ZIP

Country

Telephone

(Voice)

(FAX)

(E-mail)

Return to:

CDIAC/WDC-A

Oak Ridge National Laboratory

P.O. Box 2008 (MS-6335)

Oak Ridge, TN 37831-6335 U.S.A.

(615) 574-0390, FAX: (615) 574-2232

CDIAC@ORNL.GOV

ftp cdiac.esd.ornl.gov

URL http://cdiac.esd.ornl.gov/cdiac 